\title{
VLASOV FLUID STABILITY OF A 2-D PLASMA \\ WITH A LINEAR MAGNETIC FIELD NULL
}

\author{
Jin-Soo Kim \\ Lawrence Berkeley Laboratory \\ University of California \\ Berkeley, California 94720
}

\section{DISCLAIMER}

This report was prepared as an account of work sponsored by an agency of the United States Government. Neither the United States Government nor any agency thereof, nor any of their employees, makes any warranty, express or implied, or assumes any legal liability or responsibility for the accuracy, comp: teness, or usefu!.less of any information, apparatus, product, or process disclosed, or represents shat its uss would not infringe privately owned rights. Reference herein to any specific commerci. \& product, process, or service by trade name, trademark, manufacturer, or otherwise does not ri-essarily constitute or imply its endorsement, recommendation, or favoring by the United States Government or any agency thereof. The views and opinions of authors expressed herein do not necessarily state or reflect those of the United States Government or any agency thereof.

This work was supported by the Director, Office of Fusion Energy, U.S. Department of Energy, unde - Contract No. DE-ACO3-76SF00098. 
i i

For my parents. 


\section{TABLE OF CONTENTS}

Abstract .............................. vii

Acknowledgements .................... ix

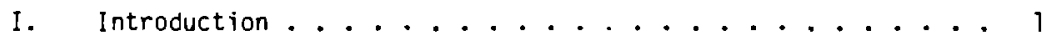

Rererences and Footnotes ................ 15

II. 2-D Equilibrium Configurate uns ........... i8

A. MHD Equilibria................. 18

B. Vlasor Equilibria................ 22

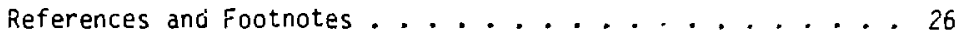

Figure Captions and Figures............. 27

III. Charged Particle Motion Near a Linear Magnetic Null. . . . 29

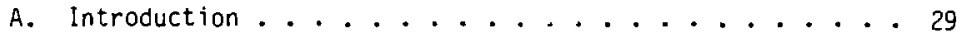

B. General Aspects of Particle Motion ......... 32

B.1 Guiding-center Motion ............. . 36

B.2 $\mathrm{J}_{\mathrm{y}}$ Conserving Particles........... 40

B.3 Summary of Expected Types of Motion ........ 44

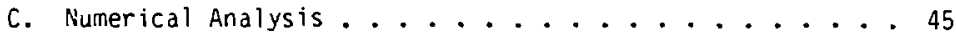

C.1 Variation with $H$ at $\epsilon=0 . \hat{c} \ldots . . . . .45$

C.2 Observed Trajectories for $\varepsilon=0.2 \ldots 47$

C.3 Scaling with $e$ at iixed H...............49

D. Summary of Orbit Analysis............ 51

E. Application to Experiments ........... 52

References and Footnotes .............. 55

Figure Captions and Figures. . . . . . . . . . 57 
IV. Vlasor Fluid Model (vf Madel). . . . . . . . . . . 74

A. Introduction ........................ 74

B. The Model .. ................... 77

c. Equilibrium. . . . . . . . . . . . . . . 79

D. Stability...................... 81

D.1 Gauge Conditions and the Variables.......... 81

0.2 The Dispersion Functional . . . . . . . . 83

0.3 Stability Theorems.................. 90

E. Calculaticn of Growth Rates . . . . . . . . . . 92

E.I The Dispersion Matrix............. 92

E.2 Properties of the VF Dispersion Operator Function . . 94

E.3 Trial Function Approach . . . . . . . . . . 97

Appendix A. Vlasov Fluid model in a Different Expression. . . 103

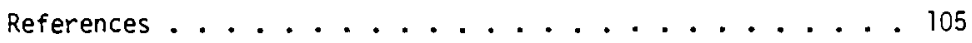

Figure Captions and Figures. . . . . . . . . . . 106

V. The Stability of a Field Reversed Configuration (FRC). . . . 108

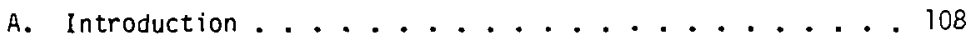

B. Linearized Ideal MHD Theory. ............ 110

C. Other Non-Kinetic Theories ........... . . . . . 19

Appendix B. The Field Reversed Experiments (FRX) at LANL. . . 123

References and Frotnotes . . . . . . . . . . . 125

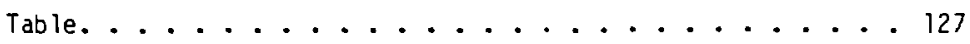

Figure Captions and Figures. . . . . . . . . . . . 128

VI. Vlasov Fluid Stability Analys is of an Elongated Z-pinch. . . 130

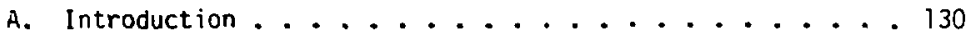

B. A VF Equilibrium in a I-pinch. . . . . . . . . . 132 
C. Character of Ion Orbits in the Z-pinch ....... 136

D. The Trial function.............. 140

E. Evaluation of Growth Rates (I) . . . . . . . 143

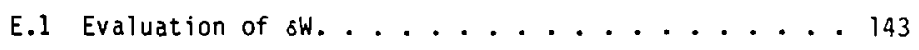

E.2 Evaluation of the Cross Term $I_{c}$....... 146

E.3 Evaluation of the Orbit Integral Term $I_{0 b} \ldots . .147$

E.4 Finding the Roots of a Dispersion Relation. .... 156

F. Evaluation of Growth Rates (II).......... I58

References and Footnotes.............. 160

Figure Captions and Figures. ................. 161

VII. Results, Discussion and Future Work. . . . . . . 167

A. Scalings of Variables of Numerical Purposes ..... 169

B. Stability Mechanism................ 178

C. Numerical Checks and Some Intermediate Steps..... 180

C.1 Transformation of Variables......... 180

C.2 Two Time Correlations of $v_{y} \ldots \ldots$. . . . . 180

C.3 Is Phase Average the Same as the Time Average

for Stochastic Orbits?............. 182

C.4 Is the Dependence of the Third Invariant of Our

Correlatior. Neak for Regular Orbits?. . . . . . 183

D. Results and Discussion............... 185

0.1 By Using the 1st VF Dispersion Functional ..... 185

0.2 Using the 2nd Formalism of the VF Dispersion

Functional................... 187

E. Future Work. ................. 191

Figure Captions and Figures................. 192 


\title{
VLASOV FLUID STABILITY OF A 2-D PLASMA \\ WITH A LINEAR MAGNETIC FIELD NULL
}

\author{
Jin-Soo Kim \\ Lawrence Berkeley Laboratory \\ University of California \\ Berkeley, California 94720
}

\section{ABSTRACT}

Vlasov Fluid stability of a 2 -dimensional plasma near an ' $O$ ' type magnetic null is investigated. Specifically, an elongated 2 -pinch is considered, and applied to Field Reversed Configurations at Los Alamos National Laboratory by making a cylindrical approximation of the compact torus. The orbits near an Elliptical ' 0 ' type null are found to be very complicated; the orbits are large and some are stochastic. The kinetic corrections to magnetohydrodynamics (MHD) are investigated by evaluating the expectation values of the growth rates of a vlasov Fluid dispersion functional by using a set of trial functions based on. ideal MHD. The dispersion functional involves fluid parts and orbit dependent parts. The latter involves phase integrat of two time correlations. The phase integral is replaced by the time integral both for the regular and for the stochastic orbits. Two trial functions are used; one has a large displacement near the null and the other away from the null. For a chosen trial function, scaling of the variables makes the growth rates depend on only two parameters: $\tilde{T}=\left(r_{L} / a\right\rangle^{2}$, where $r_{L}$ gyroradius, and a is the size of the plasma in the smaller dimension; and $\vec{k}$, the toroidal mode number if the cylinder were bent to a compact torus of major radius a. Calculations are done for $\tilde{\mathrm{k}}=1,10$ and 100 , and $\overline{\mathrm{T}}=10,0.1,10^{-3}$ and $10^{-5}$. For $\tilde{\mathrm{k}}=10$ and 
100, very reduced growth rates (more for the big displacement near the nul1) are obtained for large $\tilde{\mathrm{T}}$ while they approach those of MHD for small $\tilde{\mathrm{I}}$ values. However, for $\tilde{\mathbf{K}}=1$, the growth rates are of the same order as those of MHD. Comparison is made between those results and the Field Reversed Experiments. 


\section{ACKNOWLEDGMENT}

I would like to express my gratitude to my thesis advisor, Dr. Andrew Sessler, who guided and supported me constantly and patiently. It goes without saying that this work could not have been completed without his endless encouragement and inspiration. I would also like to thank my academic advisor, Professor Allan Kaufman. I am much obliged to him for my entire graduate work at UC Berkeley. My thanks also go to Professors Wulf Kunkel and Charles Birdsall who read this manuscript very carefully.

At the beginning of this work, I had very useful discussions with Dr. Jim Hammer. I appreciate him not only for the discussions but also for the chores he did in order to make possible my many visits to Lawrence Livermore Laboratory. The orbit analys is was done while I was at the Institute for Fusion Studies. I thank the Institute that offered me the privilege of visiting there, not to mention Dr. John Cary who arranged it entirelv. I can hardly express my thanks to him.

Many extremely useful discussions on the stability analys is were made with William Nevins. I am so grateful that he was willing to help me anytime, anywhere. I would also like to acknowledge Dr. Jim Schwartzmeier for the important discussions on the stability analys is.

Sharing from alpha to omega, I enjoyed being with post doctors and graduate students Jonnathan Wurtele, Dr. Phillipe Similon, Mr. Eliezar Rosengauss, Or. Celso Grebogi, and Steve Omahundro. My special thanks to Niels Otani who did not mind helping me out, especially with the computer code. 
Finally, I would like to acknowledge the staff and secretaries of the Magnetic Fusion Energy Division at Lawrence Berkeley Laboratory. All are so wonderful and were ready to help me all the time. I also appreciate the help from the Technical Information Division for the final typing of this manuscript.

This thesis is dedicated to my parents. The strongest support above all is their love from thousands of miles away. I deeply thank my sister, Ann Soo, who shared the agony and the joy while living with me for the past two years, and my two brothers, Kwang Soo and Won Soo. I have enjoyed discussing sciences with them from my childhood. This work was supported by the Director, Office of Fusion Energy, U.S. Department of Energy under Contract No. DE-ACO3-76SF000SI. 


\section{INTRODUCTION}

The concept of magnetic fusion energy is to confine plasma magnetically long enough until significant fusion reactions occur. Therefore, to obtain fusion energy, not only the equilibrium but also the stability should be obtained. Because of the second requirement the required applied magnetic field is normally a lot stronger than what is needed to balance the plasma pressure. Consequently $B$, the ratio of plasma pressure to applied magnetic field pressure, is very low in most cases. To enhance $B$ and thus $Q$, the ratio of output power to input power, field-reversad geometries have been proposed, where the magnetic field configuration is generated by the self-field.

Starting as early as the $195 \mathrm{j}^{\prime} \mathrm{s}$ relativistic beams were used to obtain field reversal in Astron at Livermore. ${ }^{1}$ However, the radiation from the relativistic electrons is too strong, and ion beams are now being used at Cornell. ${ }^{2}$ A field reversed system, called the Field Reversed Mirror (FRM), has been obtained ${ }^{3}$ by using a coaxial plasma gun injecting the coaxial plasma through a cusp field and eventually trapping it in a mirror well. The magnetic flux is captured as it passes through the cusp field. The field reversal has been obtained by this means in the Beta-II devict at Livermore.

A similar, but very elongated field reversed system, called field Reversed Configuration (FRC), can be created quite differently. ${ }^{4}$ A negative axial bias field is applied to a pre-ionized gas (cold plasma) in a cylinder, followed by an abrupt strong theta coil current from the main bank discharge imploding the plasma radially. The 
oppositely directed bias field is trapped inside the plasma and compressed. At tine end of compression the magnetic field lines at the plasma ends break and reconnect forming closed poloidal field loops around the plasma. All of these systems have unique advantages as fusion reactors due to the high $B$. Besides, they are compact and relatively simple, compared to Tokamaks, from the engineering point of view. Furthermore, the plasma of FRC can be translated along the axis giving more flexibility to the system.

All of the field reversed systems described above are characterized by 2-D plasma with poloidal magnetic field only. Therefore these systems have a field null line which is a circle. Because of the presence of the null, simple MHD theories are not enough to study the stability of the system. As an example, the plasmas of FRC are found, experimentally, to be stable for a long time compared to the MHD predictions. A simple present guiding center kinetic calculation also runs into difficulty near the null since the magnetic moment is not a good invariant near the null. Therefore we set ourselves the goal of explaining the long life time (compared to MHD scaling) of present FRC experiments against the MHD unstable modes.

Some of the stability, and orbits, in the 2-0 plasma with magnetic null have already been examined in astrophysics. The null appears as a sheet in geomagnetic tail ${ }^{5}$, interplanetary medium ${ }^{6}$ and so on. Most of the work [see, for example, Ref. 7] was on the tearing instability; that is, whether and how the null sheet breaks up into many null lines with ' $O$ ' type and ' $x$ ' type nulls consecutively. (The ' $O$ ' type and " $x$ " type nulls are named after the field lire configuration 
near the null.) Biskamp and Schindler ${ }^{8}$ examined the stability of a 2-D plasmi with ' $O$ ' and ' $X$ ' type nulls alternating consecutively on a plane. For modes with no variation along the null axis, $\frac{\partial}{\partial z}=0$, the energy variation shows that only modes that vanish at the ' $O$ ' point and maximize at the ' $x$ ' point are unstable. Unfortunately, similar arguments cannot be made in general for the modes where $\frac{\partial}{\partial z} \neq 0$ at this point. Therefore the stability of a FRC cannot be deduced from Schind'er's work for non zero toroidal modes.

We assume the equilibrium of FRC is a Hill's vortex ${ }^{9}$ type. This configuration has been intensively studied in hydrodynamics (for example, Ref. 10) ever since Hill introduced the toroidal vortex. It was first introduced to plasma by Shafranov. 11 The equilibria have toroidal symetry with closed poloidal field lines around the toroidal null. Of course, these satisfy the ideal MHD force balance equation, or the Grad-Shafranov equation. Yet there is, in fact, a question concerning the clost nature of the field lines. ${ }^{12}$ It has been shown that closed field $l_{i}$ s are apt to be destroyed by an infinitesimally small perturbation especially in high- 8 regions, certainly near the ' $O$ ' point. This could lead the configuration to be very complicaicd. Toroidal systems when the perturbation removes ali symmetry directions, would have field lines which are stochastic. In the presence of a strong toroidal field, $B$ is low, the extreme sensitivity of geometry is eliminated but the stochastic phenomena can be seen alsn. ${ }^{13}$ We will not consider this aspect of the subject in this work. 
The motion of a particle is limited to a region in phase space if the particle has constants of motion. If the invariants limit the particles to a finite region in a configuration space, the particle can be confined in a device. Suppose the Hamiltonian of a particle is the oniy invariant. Then an energetic particle can be confined only if the potential barrier is high enough around the region. Particles with higher energy values than the potential barrier will escape. With an additional invariant, for example angular momentum in a toroidal system with axial-symmetry, an energetic particle with higher energy vaiues than the potential barrier can be confined for a range of second invariant since the particle motion is not only constrained by conservation of energy but also by the conservation of the second invariant. ${ }^{14}$ Therefore, for an ensemble of particles with rather random initial conditions, some are absolutely confined depending on their values of invarianis. If an adiugatic invariant is found, the motion can be contained in a device for a reasonably long time.

Absolute confinement of individual particles in a device does not inply plasma confinement. Collective phenomena, an instability, may cause the system to be unstable. Therefore stability has been an important issue in the study of plasma. In a collision-free limit, the study of the linear kinetic stability requires knowledge of the orbits. When the orbits are integrable (a11 the orbits are regular with the same number of invariants as the dimension of the system) the kinetic treatment of orbits are simple in concept ${ }^{15}$ but complexity usually follows upon implementation. Simple expressions of orbits could reduce the complexity in the kinetic calculation by a great deal. When 
the orbits are stochastic then the plasma stability is extremely difficult. ${ }^{16}$ There is no way of expressing the orits analytically in any manner. Orbits in field reversed systems are very complicated due to the magnetic null surrounded by strong field gradient and curvature. Conservation of magnetic moment breaks down and the guiding center theory does not apply. A significant portion of the orbits are stochastic. Even the regular orbits do not have a simple expression which describes the motion. As a result the understanding of the stability of such a system is still in a primitive stage. To analyze the stability of this system, new techniques taking into account the stochastic orbits as well as large orbits have to be developed.

For this purpose, we first investigated the orbits of particles near a one-dimensional magnetic null, specifically near a straight ' $O$ ' type field null axis. A linear geometry is chosen since it is simple and can be applicable to a toroidal null if the region of interest is small enough that the lowest-order, linear terms in the Taylor expansion of the field near the null is valid. If we further assume that the electric field is negligible, the Hamiltonian can be reduced by scalings to a two-dimensional Hamiltonian in an effective potential. Depending on the sign of $P_{z} / e \psi$ of the original Hamiltonian, where $P_{z}$ 
is the canonical momentum in the axial direction, $e$ is the charge and $\psi$ is the flux function, the effective potential is either a quartic well $\left(0=-1\right.$ where $\sigma$ is the sign of $\left.\left(P_{2} / e \psi\right)\right)$ or a quartic well with a local maximum at the null $(\sigma=+1)$.

Adiabatic invariance analysis and numerical surface of section analysis are used to study the behavior of particles in this system. The behavior of orbits are examined first analytically, based on adiabatic invariance theory, and then refined by numerical study. The method used in the numerical analysis is mainly the surface of section plots. This technique is used in nonlinear studies [see, for example, Ret. 17].

The results of this study are conveniently expressed in terms of the two parameters for a given $\sigma: \varepsilon$ parameterizes the elongation of the ellipse of a flux surface ranging from $\varepsilon=0$ of a slab geometry to $\varepsilon=1$ of a circular flux surface and $H \equiv(1 / 2)\left(2 \mathrm{mh} / \mathrm{P}_{z}^{2}\right)$ which is twice the particle Hamiltonian, $2 \mathrm{~h}$, measured by the local potential at the null $P_{2}^{2} / 2 m$. (1) For $\sigma=-1, H>0.5$ should be satisfied. For all parameters of $H$ and $c$ the observed motion is regular. When $\varepsilon=1, P_{\theta}$ is the additional inyariant. When $\varepsilon \ll 1$, the adiabatic invariant is $J_{Y}$, the action in the minor radial direction. An apparent $\omega_{x} / \omega_{y}=2$ resonance appears only with little ergodic behavior. (2) For $0=+1$, particles with $H>0$ are allowed. Roughly $\varepsilon-H$ space is divided into three regimes. (2a) for $H \gg 0.5$ the orbit behavior is qualitatively the same as those of $a=-1$. This is expected since the effect of $\sigma$ is negligible for particles with high $H$, although the resonance around c $=0.5$ brings apparent ergodic behavior. (2b) For intermediate 
values, $e^{3}<H<0.5$, nearly all orbits are stochastic. The stochastic behavior occurs due to the random jumps of the invariant values at the magnetic well. (2c) For very small values, $H \ll \varepsilon^{3}$, particles are regular with the magnetic moment $(\mu)$ as the third invariant. Guiding center theory is valid in this regime.

Applying our results to the plasma of FRC experiments at $\operatorname{LANL}^{4,5}$ we find that ions near the magnetic null are mostly regular, ions near the plasma edge are mostly stochastic. As the size of plasma increases from $F R X-B$ to $F R X-C$ the portion of stochastic orbits increase. As the temperature increases more particles become regular. At any event the $\mu$-conserving orbits are negligible.

The results of this study are consistent with and complement previous work on particle orbits. Larabee and Lovelace ${ }^{18}$ studied ion motion in a more realistic geometry and found primarily regular orbit behavior, agreeing with our result, On the other hand $F_{i n n}{ }^{19}$ found stochasticity with $\sigma=-1$ ions. However, notice that his field does not reduce to our geometry near the null. (The previously mentioned Taylor expansion does not apply in his field). Orbits of extreme values of $\varepsilon$ have also been investigated. Wang and Miley ${ }^{20}$ studied ion orbit behavior in FRM where the geometry is characterized by $\varepsilon=1$. Sonnerup ${ }^{21}$ studied the property of $J_{Y}$ invariance of particles near a null sheet where $\varepsilon=0$. The present study agrees with these results but goes further by discussing other regions of parameter space as wel1. Mynick's ${ }^{22-24}$ work on adiabatic invariant theory is extended by studying in a wider farameter range and finding the onset of stochasticity. 
With enough knowledge of the single particle orbits, we are ready to examine the collective phenomena of the system. We are now convinced that the collective phenomena, stability, near the null cannot be explained adequately by simple MHD analyses. As an example the plasmas of a field reversed configuration are found to be stable for many Alfuén transit times, the characteristic time for an Alfvén wave to travel once around flux surface. On the contrary all the present MHD type simple calculations predict linear exponential growth time as an order of one Alfuén transit time. The calculations include ideal linear [see, for example. Ref. 25] and nonlinear 26 MHD energy variational analysis, resistive ideal $\mathrm{MHD}^{27}$ and double adiabatic ${ }^{28}$ calculations. Ion orbit effect on the stability has been attempted by making a small-gyroradius expansion. However, this theory breaks down near the nul1, since the magnetic moment is not a constant of motion. 29 Here, we present a method of treating the large orbits, both regular and stochastic, in stability analysis.

Our kinetic analysis is based on the Vlasov Fluid (VF) model. 30 It is a low frequency model of a fully ionized nlasma in which electrons are treated as a massless, pressureless fluid and ions are treated as collisionless. This choice is made since the present FRC experiments are operated in a regime where electron collision time is very short and that of ions is more or less longer than the MHD time scaling, the Alfvern transit time. Furthermore the model gives the same force balance equation as that of ideal MHC for a class of ion equilibrium distributions. This property is useful if one is interested in studying the orbit effects on MHD. In fact, whether one 
chooses the Vlasov fluid model or some other kinetic model such as twoVlasov analysis is of secondary importance; the primary problem is the treatment of complicated orbit effects.

No matter which model one chooses, the kinetic calcuation requires a time integral over the unperturbed orbits. If there are some scalings, such as fast time or short length scalings, one may be able to carry out the time integral by making appropriate approximations. However, we are interested in a regime where no scalings exist. Futhermore, some of the orbits are stochastic.

In order to treat those orbits, we first describe a VF dispersion functionat. It is obtained by the following procedure: (1) Linearize the force balance equation. Let the perturbed quantity be $\underline{\xi}$. (2) Then apply a scalar product with $\xi^{\star}$ and integrate over the configuration space to form a VF dispersion functional which is a function of $\xi^{\star}$ and $\underline{\xi}$ with $w$ as a parameter. Such a functional always reduces to the original eigenvalue problems by varying $\underline{\xi}^{*}$ treating it independently with respect to $\underline{\xi}$. If we do the same thing by expressing $\underline{\xi}^{\star}$ and $\underline{\xi}$ in terms of $n$ basis functions, we hạve $n$ homogeneous equations instead. If the $n$ basis functions form a complete set, the solution of the $n$ equations would be the solution of the original eigenvalue problem. The dispersion relation is obtained by setting the determinant of the matirx obtained from the n-homogeneous equations to zero. It is the dispersion matrix ${ }^{31,32}$ and the $(i, j)$ element has the same form of a dispersion functional but with $\underline{\xi}^{\star}$ and $\underline{\xi}$ replaced by the $i^{\text {th }}$ and $j^{\text {th }}$ basis functions of the corresponding vectors. In principle we obtain an exact dispersion relation by this procedure if we take enough basis functions. There 
are two steps involved. First step: Evaluate each element of the matrix. If we realize that each element requires detailed orbit information, this is the major siep. Second step: Solve the matrix: In a practical calculation, one may truncate the number of basis functions and approximated dispersion functions can be obtained. Then the question is how many basis functions are needed and whether it is practical. The question can be answered only after the first step. This work is devoted to the first step to find out whether our method is practical in soiving a big matrix probiem.

The first step itself has some physical meaning. Suppose we know the eigenvector on some physical grounds, we obtain tise corresponding eigenvalue without solving the whole matrix problem. Also, if we are interested in the expectation eigenvalues for some specific form of vectors, or trial functions, step 1 is enough. Furthermore the VF model has the same equilibrium condition and the same marginal stability condition as MHO for a class of ion distributions. In this case, if we use eigenvectors of ideal MHD as trial functions, the expectation values give the same marginal condition and give corrections away from the marginal point.

We study the VF stability from two different expressions (Refer Chap. IV. See Eqs. (4.29) and (4.31)). The VF dispersion functional (I) is composed of three terms, the ideal MHD energy variational of incompressible displacements, the orbit integral terin arid the left-over fluid term. The ideal MHD term and the left-over fluid term can be evaluated without any difficulties. The second expression has MHD plus the orbit corrections, thus the kinetic effect is more explicit. 
Whatever the formalism is, the orbit term involves a six-dimensional phase space integral and the time integral over the unperturbed ion trajectories. Time and the phase variables are independent, thus interchanging the order of integration the orbit term becomes a time integral of a two time correlation function. Since particles obey autonomous dynamics the correlation function is independent of the initial time but only depends on the time difference of the functions defined. ${ }^{33}$ Therefore it is suggestive to do the time-Fourier transformation with respect to the time difference, the lag time. Then the time integral becomes trivial. Thus the orbit integral term is transformed to an integral of dynamical power spectral densities. ${ }^{19}$

The dynamical spectral densities are obtained by choosing some number of initial conditions and following them for some time to pick up all the interesting spectra. The initial conditions are chosen regularly in $\mathrm{H}$ and $P_{Z}$ space and randomly in the remaining 4-D space. Stochastic orbits are efficiently treated, but regular orbits are also treated this way assuming that the correlation function is a weak function with respect to the third invariant. This could be a strong assumption but numerical tests indicate that our correlation function does satisfy the assumption approximately. Numerically, spectral densities are evaluated at discrete values of frequencies, a dummy variable to be surmed over. Because of the presence of the dummy variable appearing in the denominator, the discreteness brings extra non-physical roots to the dispersion relation. This problem can be removed by the use of the Nyquist Diagram. ${ }^{34}$ By plotting Nyquist Diagrams for 
many different frequencies, a good guess at the physical solution is obtained to be used as an input for a root solver.

This method is applied to study the stability of a FRC at LANL by approximating it as an elongated $2-p i n c h$. The linear geometry is simple, yet it contains the main character of the FRC such as closed field lines, MHD instabilities, large orbits and particle stochasticity. Of course, exact comparisons cannot be made since the toroidal effect could be important for kinetic stability of a compact toroidal system. Nevertheless, the effect due to the large orbits and stochasticity can be studied. We investigate the kinetic corrections to MHD due to the large orbits and stochasticity by evaluating the expectation values of the growth rates of a VF dispersion functional of a set of trial functions based on ideal Mho. In particular, we choose two trial functions, one localized near the null (trial function I) and the other localized away from the null (trial function II).

For a chosen trial function, the expectation value of the growth rate depends on oniy two parameters, if we scale the variables to eliminate all the unnecessary parameters. The two parameters are $\tilde{T} \equiv\left(\frac{r_{L}}{a}\right)^{2}$, where $r_{L}$ is the gyro-radius and $a$ is the size of the plasma in a smaller dimension; and $\tilde{k}$, which is the toroidal mode number if the cylinder were bent to a compact tcrus of major radius a. Growth rates are evaluated for $\overline{\mathrm{k}}=1,10$ and 100 and $\tilde{\mathrm{T}}=10,0.1$, $10^{-3}$ and $10^{-5}$. The plasmas of FRX-B at LANL correspond to $\tilde{T}=0.1$. When the first formalism Eq. (4.29) is used by following each orbit only up to an MHD growth time, the growth rates remain of the same 
order as those of MHD for almost all the parameters we tested. The reason is that the real frequency of the solution is smaller than the smallest frequency that is accurately obtained by following the orbits for about MHO growth time $\left(T_{M H O}\right)$ which is $1 / Y_{M H O}$. (Notice that for VF model, the real part of w approach to zero as the growth rate does.)

Instead of trying to get better spectral densities near zero frequency which can be done by tracing the orbits for a longer time, we prefer studying the second formalism Eq. (4.31) since it gives the kinetic correction explicitly, and thus high accuracy may not necessary. When the second expression is used, very reduced growth rates are obtained for $\tilde{k}=10$ and 100 for $\widetilde{T}=0.1$ and 10 . And the growth rates approach those of MHD values as $T$ decreases $\left(10^{-3}\right.$ and $\left.10^{-5}\right)$. In fact, we traced orbits for about 10 times of $T_{M H D}$ to obtain better results for $\widetilde{k}=10$. For $\widetilde{k}=1$, the growth rates are the same order as those of MHD for ail $\tilde{T}$ values tested even when we followed each orbit 10 times longer than $T_{M H O}$.

Similar phenomena are observed throughout the parameters for the secand trial function II. The only difference is that the growth rates are somewhat less reduced for high $\tilde{\mathrm{T}}$ values than those of trial function 1 .

The reduced growth rates for low $\tilde{k}$ values may be obtained by one or some of the following; (1) Follow the particles much longer to get accurate spectrial densities near zero frequency. (2) Include the terms neglected in the second dispersion expression. (3) Take into account the toroidal effect. 
The outline of the thesis is as follows: In Chap. II, we study MHD and kinetic equilibrium configurations of a 2-D plasma in general. In Chap. III, the motions of charged particles in 2-D near a linear magnetic field nuli $7^{35}$ are studied in detail. We first analyze the motion by invariant theory to predict some types of regular orbits and to estimate the onset of stochasticity. This prediction is supported and refined by numerical observations. The results are applied to characterize the ion orbits of a plasma in FRC. In Chap. IV we discuss the Vlasov fluid model. A VF dispersion functional and the dispersion matrix are described. Also correlation and dynamical spectral densities are introduced to re-formulate the robit dependent term in the VF dispersion functional. We briefly mention the basic framework of Symon, Seyler and Lewis ${ }^{31,32}$ on the VF model as a comparison in Appendix A. Chapter $V$ summarizes the present non-kinetic stability calculations on FRC. A brief summary of the experiment is presented in Appendix B. In Chap. VI, the method is applied to study the stability of a FRC at LANL by approximating it as an elongated Z-pinch. Finally the results and the numerical checks along the way are presented in Chap. VII. Future work is also discussed.

My main contributions are described in Chapters III, VI, and VII. Part of Chap. IV is original. I have made a clear distinction between my work and that of others at the end of the Introduction to Chap. IV. Each chapter is organized to be independent except Chaps. VI and VII which depend upon all of the previous chapters. References and figures for each chapter are at the end of each chapter. 


\section{REFERENCES AND FOOTNOTES (CHAP. I)}

1. N. C. Christofilos, Proceedings of the 2nd International Conference on the Peaceful Uses of Atomic Energy (United Nations, Geneva, Switzerland, 1958), 32, 279 and N. C. Christofilos, Nucl. Fusion Supplement, Part I, 159 (1962).

2. H. H. Fleischman and T. Kammash, Nucl. Fusion 15, 1143 (1975).

3. E. V. Anderson, S. P. Auerbach, H. L. Berk. J. K. Boyd, T. A. Brengle, J. A. Byers, B. I. Cohen, W. C. Condit, J. C. Eddieman, R. P. Freis, E. H. A. Granneman, J. H. Hammer, C. W. Hartman, J. Killeen, B. McNamara, W. A. Newcomb, L. D. Pearlstein, M. G. McCoy, D. S. Prono, J. M. Sayer, D. D. Schnack, D. Schumaker, J. W. Shearer, A. C. Smith, Jr., J. Taska, W. C. Turner, D. E. Driemeyer, G. H. Miley, E. C. Morse, E. MrColl and H. Weitzner. Proceedings of the 8 th International Conference on Plasma Physics and Controlled Nuc?aar Fusion Research, (Brussels, 1980), Vol. I, IAEA, Vienna, 1981.

4a. A. G. Es'Kov, K. Ku. Kurtmallaev, A. P. Kreshuk, Ya. N. Lank in, A. I. Molyutin, A. I. Markin, Ya. S. Martyuskov, B. K. Mirov, M. M. Orlov, A. P. Proxletsov, V. N. Semyenov, and Yu. B. Sosunov, in Plasma Physics and Controlled Nuclear Fusion Research (IAEA, Vienna, 1978), Vor. II, p. 187.

4b. W. T. Armstrong, R. K. Linford, J. Lipson, D. A. Platts, and E. G. Sherwood, Phys. Fluids 24, 2068 (1981). 
4c. R. E. Siemon, W. T. Armstrong, R. R. Bartsch, R. E. Chrien, J. C. Cochrane, R. H. Kewish, P. C. Klingner, R. K. Linford, J. Lipson, K. F. McKenna, D. J. Rej, E. G. Sherwood, and M. Tuszewski, presented at the Ninth International Conference on Plasma Physics and Controlled Nuclear Fusion Research, Baltimore,. Maryland, 1982, Paper IAEA-CN-4I M-2-1.

5. N. F. Ness, J. Geophys, Res. 70, 2989 (1965).

6. 3. M. Wilcox and N. F. Ness. J. Geophys, Res. 70, 5793 (1965).

7. K. Schindler, D. Pfirsch and H. Wobig, Plasma Phys, 15, 1165 (1973).

8. D. Biskamp and K. Schindler, Plasma Phys. 13, 1013 (1971).

9. M. J. Hill, Phil. Trans. Roy. Soc. Ser. A. Pt. I, C/XXXV 213 (1894).

10. H. Lamb, Hydrodynamics (Dover, New York, 1932).

11. V. D, Shafranov, Sov. Phys. JETP 6 , 545 (1958).

12. A. H. Boozer, Nucl. Fusion 18, 1663 (1978).

13. D. W. Kerst, Plasma Phys. $\underline{4}, 253$ (1962).

14. R. V. Lovelace, D. A. Larrabee and H. H. Fleischmann, Phys. Fluids 22, 701 (1979).

15. A. N. Kaufman, Phys. Fluids 15, 1063 (1972).

16. B. V. Chirikov, Phys. Rep. 52, 263 (1979).

17. M. Henon and C. Heiles Astron. J. 69, 73 (1964).

18. D. A. Larrabee and R. V. Lovelace, Phys. Fluids 23, 1436 (1980).

19. J. M. Finn, Plasma Phys. 21, 405 (1979).

20. M. Y. Wang and G. H. Miley, Nucl. Fusion 19,39 (1979).

21. B. U. 0. Somnerup, J, Geophys. Res. 76,8211 (1971). 
22. H. E. Mynick, Phys. Rev. Lett. 43,1019 (2979).

23. H. E. Mynick, Phys. Fluids 23, 1888 (1980).

24. H. E. Mynick, Phys. Fluids 23, 1897 (1980).

25. W. A. Newcomb, Phys. Fluids, 23, 2296 (1980).

26. Yu A. Drezin and E. P. Sololcy, Sov. J. Plasma Ph_.. Z, 516 (1981).

?7. A.I. Shestakov, J. Killeen and D.D. Schnaci: J. Comp. Phiys. 46, 65 (198?:.

28. J. H. Hammer, privace commnication.

29. C. E. Seyler and D. C. Barrier, Phys. Fluids 24, 1989 (1981).

30. 3. P. Freidberg, Fnys. Fluids 15 : 1102 (1972).

31. H. R. Lewis and K. R. Symon, J. Yath. 'hys, 20413 (1979).

32. K. R. Symon, G. E. Seyler, anci H. R. !ewis, J. Plasma Phys. 2?, paper I, II and III, (1982) pp. 13-53.

33. J. M. Finn and R. N. Sudan, Nucl. Fusion 22, 1443 (1982).

34. N. A. Krall and A. W. Trivelpiece, Principles of Plasma Physics (MCGraw-Hill, New York, 1973), Chap. 9, Secs. 6 and 10.

35. J.S. Kim and J.R. Cary, Phys. Fluios 26, 2167 (1983). 


\section{CHAPTER II}

\section{2-D EQUILIBRIUM CONFIGURATIONS}

Many plasmas of interest, from fusion energy experiments to solar systems, have a symmetry such as translational, toroidal or nelical. Such systems are described by 2-Dimensional configurations, since a coordinate and its associated momentum can be transformed away, or held constant, because of the symmetry. In this chapter we will discuss the equilibria of such systems first by ideal MHD theory and then by Vlasov Theory. The equilibria of the VF model will be discussed in Chap. IV.

\section{A. 2-D Ideal MHD Equilibria}

The basic ideal MHD equilibrium equations for a static plasma are

$$
\begin{aligned}
& \underline{\nabla} P=\frac{l}{c} \underline{J} \times \underline{B}, \\
& \underline{J}=\frac{c}{4 \pi} \underline{\nabla} \times \underline{B}, \\
& \underline{\nabla} \cdot \underline{B}=0 .
\end{aligned}
$$

From the first equation of Eq. (2.1), it is clear that the magnetic field lines lie on a constant pressure surface since $\underline{B} \cdot \underline{\nabla} P=0$. To help our understanding first consider a constant pressure surface of a torus. We now know that the magnetic field lines should be on this torus. They either cover the torus ergodically or close themselves after a finite number of toroidal circuits. The latter happens when 
the ratio of the toroidal angular velocity to the poloidal angular velocity is a rational number, in which case it is called a rational surface. All toroidal systems in general have rational surfaces, but the number of such surfaces is of measure zero compared to the ergodic surfaces.

When a magnetic field line covers a torus ergodically, there is a well defined surface, which is called a magnetic surface. A magnetic surface is a surface formed by a single magnetic field line. The flux through any cross sections of a magnetic surface is constant because all the magnetic field lines inside of the surface must remain inside the surface since the field is tangential to the surface. Hence all magnetic surfaces are flux surfaces.

For closed field line systems (e.g. Z-pinch, FRC ...) or systems characterized by field lines extended to infinity (e.g. mirror machine) we cannot talk about magnetic surfaces in general. Also, there is no natural way of uniquely determining flux surfaces. However, using the fact that a constant pressure surface is also a flux surface (due to $\underline{B} \cdot \underline{\nabla} P=0$ ) we can conveniently specify a flux surface as a surface of constant pressure. When we say flux surfaces of such systems, it will usually mean the surfaces of constant pressure unless specified otherwise.

When there is a symmetry in the system, it is possible to reduce the equilibrium equations to a single partial differential equation with one unknown by introducing a stream function $\psi$. (The stream functicn is related to the component of a vector potential in the 
ignorable coordinate.) First we will present the reduction scheme of an axi-symmetric toroidal system. Choose a cylindrical coordinate $(R, Z, \phi)$ as shown in Fig. 2.1. Because of the toroidal symmetry, $\frac{\partial}{\partial \phi}=0$, and the potential magnetic field may be expressed in terms of toroidal component of the vector potential $A_{\phi}$ or equivalently the stream function $\psi$ alone.

$$
\begin{aligned}
\underline{B} & =\underline{\nabla} \times\left(A_{\phi} \hat{\phi}\right)+B_{\phi} \hat{\phi} \\
& =\frac{1}{R} \nabla \psi \times \hat{\phi}+B_{\phi} \hat{\phi} \\
& =\frac{1}{R} \frac{\partial \psi}{\partial Z} \hat{R}-\frac{1}{R} \frac{\partial \psi}{\partial R} \hat{Z}+B_{\phi} \hat{\phi},
\end{aligned}
$$

where $\psi=\mathrm{RA}_{\phi}$.

It is easily seen that the stream function $\psi$ is directly related to the poloidal flux $\psi_{p}: \psi_{p}=2 \pi \psi$. From $\underline{J}=\frac{C}{4} \underline{\nabla} \times B$, the current density $\underline{J}$ is readily expressed in terms of $\psi$.

$$
\underline{J}=\frac{C}{4 \pi}\left[-\frac{1}{R} \Delta^{\star} \psi \hat{\phi}+\frac{1}{R} \nabla\left(R B_{\phi}\right) \times \hat{\phi}\right],
$$

where $\Delta^{*}$ is the elliptic operator defined by:

$$
\Delta^{*} \psi \equiv R \frac{\partial}{\partial R}\left[\frac{1}{R} \frac{\partial \psi}{\partial R}\right]+\frac{\partial^{2} \psi}{\partial z^{2}} .
$$

Now we work on the force balance equation component by component. The $\underline{B}$ component of the equation is $\underline{B} \cdot \underline{\nabla}=\frac{1}{C} \underline{B} \cdot \underline{J} \times \underline{B}=0$. From the explicit expression of $\underline{B}$ (Eq. 2.2) and $\underline{J}$ (Eq. 2.3) the condition 
$\underline{B} \cdot \underline{J} \times \underline{B}=0$ becomes $\phi \cdot \underline{\nabla} \psi \times \underline{\nabla P}=0$ which indicates that $P$ is a function of $\psi$ only:

$$
P=P(\psi) \text {. }
$$

Likewise the $\underline{J}$ component of the force balance equation is $\underline{J} \cdot \underline{\nabla}=\frac{1}{c} \underline{J} \cdot \underline{J} \times \underline{B}=0$ which yields $\hat{\phi} \cdot \underline{\nabla} \times \underline{\nabla}\left(R B_{\phi}\right)=0$. Hence $R B_{\phi}$ is also a function of $\psi$ only.

$$
R B_{\phi}=I(\psi)
$$

It turns out that $I(\psi)$ is proportional to $I_{P}$ where $I_{P}$ is the poloidal current passing through the surface bounded by $R=$ constant and $Z=0$. Eventually, the force balance equation is reduced to

$$
-\Delta * \psi=4 \pi\left[R^{2} P^{\prime}(\psi)+I I^{\prime}(\psi)\right]
$$

where the prime indicates the derivative with respect to the argument 4. This is called the Grad-Shafranov equation. It is a second order nonlinear partial differential equation describing axisymmetric toroidal equilibria. The term on the left hand side of Eq. (2.7) represents $\underline{J}_{\phi} \times \underline{B}_{p}$ while II'( $\psi$ ) on the right hand side of $E q$. (2.7) represents $J_{p} \times B_{\phi}$ where subscript $\phi$ indicates the toroidal component of the quantity and the subscript $P$ indicates the poloidal component of the quantity.

The equilibrium of an axisymmetric toroidal system is determined by (1) specifing the two free functions $P(\psi)$ and $I(\psi)$ with boundary conditions or externally imposed constraints on and (2) inverting the elliptical operator $\Delta^{\star} \psi$ to determine $\psi=\psi(R, Z)$. 
For straight cylindrical systems there is also at least one ignorable coordinate $\frac{\partial}{\partial z}=0$ due to the translational symmetry (say in $z$ ). In using Cartesian coordiriates $(x, y, z)$

$$
\underline{B}=\frac{\partial \psi}{\partial y} \hat{x}-\frac{\partial \psi}{\partial x} \hat{y}+B_{z} \hat{z},
$$

where $\psi=A_{z}$ which is the axial component of the vector potential. Then following the steps used previously we can conclude easily

$$
\begin{aligned}
& P=P(\psi), \\
& B_{z}=B_{z}(\psi),
\end{aligned}
$$

and obtain

$$
-\nabla^{2} \psi=\left[P^{\prime}(\psi)+B_{2} B_{2}^{\prime}(\psi)\right] 4 \pi
$$

The MHD equilibria of plasmas with helical symmetry also have the properties that we have discussed. The expression equivalent to the Grad-Shafranov equation is available in Bateman ${ }^{2}$ on Page 70.

\section{B. 2-D Vlasov Equilibria}

To study the character of plasmas more accurately, and in detail, we have to go back to kinetic theory. On a time scale shorter than the collision time, the properties of collective phenomena of plasmas are well described by the collisionless Boltzmann or Vlasov equation. In that description, the interaction between the particles is only through the averaged electric and magnetic fields produced at a point 
$\underline{x}$ by the particles. These fields must be computed self-consistently through Maxwell's equations.

A steady state solution of the vlasor equation is not unique. One procedure for generating a solution of time independent form of the Vlasor equation is by finding time independent constants of motion of the particles.

The constants of motion describing the motions of particles are functions of the variables $\underline{x}, \underline{v}$ and possibly $t$. Let $c^{i}(\underline{x}, \underline{y}, t)$ be a constant of motion. Then by definition $\frac{d C}{d t}(\underline{x}, \underline{y}, t)=0$ and thus any function $F(\underline{x}, \underline{v}, t)$ which is a function of constants of motion only is a solution of vlasov equation since

$$
\frac{d f}{d x}(\underline{x}, \underline{v}, t)=\sum_{i} \frac{\partial f\left(c_{1}, c_{2}, \ldots\right)}{\partial C_{i}} \frac{d c_{i}}{d t}=0 .
$$

If the $C_{i}$ 's are time independent constants of motion, we have a steady state solution of the vlasov equation.

Consider a 2-D plasma with translational symmetry in the $z$ direction. Two constants of motion are easily found. They are the energy and the z-component canonical momentum of the particles. Hence, a class of the vlasor equilibria can be expressed symbolically as

$$
f_{0}(\underline{x}, \underline{v})=F_{0}\left(H, P_{z}\right)
$$

where introduced $f_{0}$ and $F_{0}$ to indicate the dependence of the arguments. The assumption behind the choice of the equilibrium distributions as functions of $\mathrm{H}$ and $\mathrm{P}_{Z}$ only is that the orbits are ergodic. 
If not, non-overlapping regions of orbics in a phase volume of constant $H$ and $P_{z}$ are treated with equal weight statistically.

To find an equilibrium configuration we have to employ Maxwell's equations. By choosing a Lorentz gauge, $\underline{\nabla} \cdot \underline{A}=0$, the steady state Maxwell's equations are:

$$
\begin{aligned}
& -\nabla^{2} \underline{A}_{0}=\underline{J}_{0}\left(\underline{A}_{0}, \Phi_{0}\right), \\
& -\nabla^{2} \Phi_{0}=\theta_{0}\left(\underline{A}_{0}, \Phi_{0}\right),
\end{aligned}
$$

with $\rho_{0}=\sum$ e $\int F_{0} d \underline{v}$,

and $\underline{u}_{0}=\sum$ e $\int \underline{v F}_{0} d \underline{v}$.

Notice that $\underline{J}_{0}$ and $o_{0}$ depend on $\underline{x}$ only through $\underline{A}_{0}(\underline{x})$ and $\phi_{0}(\underline{x})$, which is obvious from the fact that $f_{0}(\underline{x}, \underline{v})=F_{0}\left(H, P_{2}\right)$ where

$$
H=\frac{M v^{2}}{2}+e \Phi(\underline{x}) \text { and } P_{z}=M v_{z}+\frac{1}{c} e A_{0}(\underline{x}) \text {, }
$$

and the sumation is over different species. For a 2-D plasma only the component along the ignorable coordinate of $\underline{A}_{0}$ is necessary, namely $\underline{A}_{0}=A_{0} \hat{z}$.

A class of equilibrium configurations is obtained by inverting the Laplacian to determine

$$
-\frac{2}{A_{0}}=J_{0}\left(A_{0}\right) .
$$


With an imposed boundary condition on the boundary $\partial D$ of a domain $D$ in the $x, y$ plane this is a well defined problem. One convenient choice could be $A_{0}$ and $\Phi_{0}$ constant on $2 D$.

Once $A_{0}(\underline{x})$ is determined $\Phi_{0}(\underline{x})$ is obtained by the quasi-neutrality assumption.

By choosing a specific form of $F_{0}\left(H, P_{z}\right)$, the charge densities of the electrons and ions can be evaluated explicitly as functions of $A_{0}$ and $\Phi_{0}$. Hence the quasi-neutrality condition

$$
\rho_{0}^{i}\left(A_{0}, \Phi_{0}\right)+\rho_{0}^{e}\left(A_{0}, \Phi_{0}\right)=0
$$

determines $\Phi_{0}$ as a function of $A_{0}$. Thus, we have found a vlasov equilibrium. 
REFERENCES AND FOOTNOTES (CHAP. II)

The major references used for the discussions of 2-D MHD equilibrium are:

1. J. P. Freidberg, Rev. Modern Phys. 54, 801 (1982).

2. G. Bateman, MHD Instabilities (MIT Press, Cambridge, Massachusetts, 1980), Chap. 4

3. V. D. Shafranov, Reviews of Plasma Physics, Vol. 2, (Consultant Bureau, New York, 1966) p. 103.

and those of 2-D kinetic equilibria are:

4. K. Schindler, Plasma Phys. $\underline{15}, 1165$ (1973).

5. G. Schmidt, Physics of High Temperature Plasmas, (Academic Press, New York, 1979), p. 66-68. 


\section{FIGURE CAPTION}

Fig. 2.1 A cylindrical coordinate of a toraidal system. 


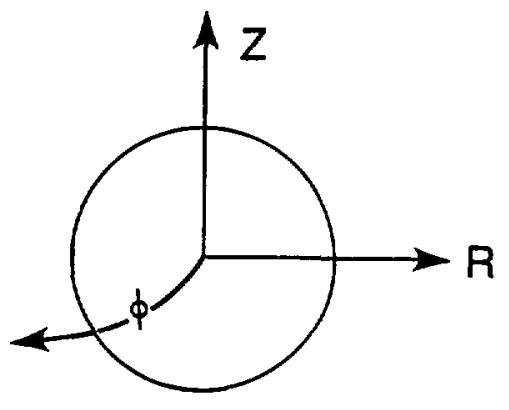

XBL 8312-6714

Figure 2.1 
CHAPTER III

CHARGED PARTICLE MOTION NEAR A LINEAR MAGNETIC NULL

\section{A. Introduction}

Systems with field nulls have drawn special attention recently. One main reason is that the field reversed systems have unique advantages as feasible fusion reactors. Nevertheless the stability of those systems is poorly understood.

For example, experiments on Field Reversed Configurations (FRC) indicate that these systems are stable for many Alfvén transit times, ${ }^{1-3}$ the characteristic time for an Alfvên signal to travel once around the flux contour. This contradicts the present fluid calculations (see Chap. $V$ and its references) which indicate the exponential growth time on the order of one Alfvén transit time. Kinetic calculation seems to be necessary to resolve the contradiction.

Kinetic analysis depends heavily on the detailed motion of particles. Thus as a preliminary to the kinetic stability analysis we set the goal of this chapte. to characterize the orbits of particles in a system with a magnetic null. For simplicity, we study the orbits near a linear magnetic null. The orbits near a toroidal null are also deduced from the study of the linear null since the $l$ inear null retains the toroidal systems' essential pr-erties: the null, the closed field lines and particle stochasticity.

The main advantage of choosing the linear system is that the canonical momentum in the axial direction $P_{z}$ can be transformed away 
but there are two situations corresponding to the sign of the $P_{z} / e \psi$ where $\psi$ is the flux function. The effective potential of the reduced Hemiltonian is either a simple quartic well (we introduce a symbol $\sigma$ to distinguish the sign of $P_{z}$ and let $a=-1$ for this case) or a quartic well with a local maximum at the null $(\sigma=+1)$.

The motion of particles is first analyzed analytically in terms of adiabatic invariants and then tested numerically mostly by surface of section plots. We characterize the motion in terms of $H$ and $\varepsilon$ where $H$ is half the energy of a particle measured by the incal potential at the null and $\varepsilon$ parameterizes the elongation of flux surfaces. In general there are three types of motion (refer Fig. 3.15). (i) For $H \gg 0.5$ the orbits are regular $(H=0.5$ is the local maximum potential energy at the null for $\sigma=1$ and $\therefore$ the lower bound of the energy for $\sigma=-1$ ). When $\varepsilon$ is close to unity (circular flux surfaces), the adiabatic invariant is the canonical angular momentum associated with the azimuthal symmetry, and when $\varepsilon \ll 1$, it is the action in the minor axial direction $\left(J_{Y}\right)$ due to the frequency disparity between the oscillations in the minor and the major directions. This $J_{y}$ conserving orbits are either figure eight type $(\sigma=+1)$ or betatron type $(\sigma=-1)$. Significant resonance structure appears only at $\varepsilon=0.5$ but even in this case very little ergodic behavior is onserveu. (2) For very low energy values, $H \ll \varepsilon^{3}$, guiding-center motion conserving magnetic momen $(u)$ is cbserved. (3) For intermediate values $\varepsilon^{3}<H<0.5$ nearly all orbits are stochastic. The stochastic behavior occurs by the $r$ andom jumps in values of $J_{\psi}$ or $\mu$ whichever is more appropriate 
when the particle passes the weak magnetic field region around the tips of the elliptical flux surface.

Applying the result to the FRC experiments at LANL, ${ }^{2,3}$ we find that the ion orbits near the null are regular, $J_{y}$ conserving, and most ion orbits near the edge are stochastic. As the temperature of ions increases, more orbits are regular and as the size of the plasma increases (from $F R X-B$ to $F R X-C$ ) more orbits are stochastic. The $\mu$ conserving orbits are negligible in any case.

The results of this study are consistent with and complement previous discussions of particle orbits. Finn ${ }^{4}$ observed stochasticity" for particles with $a=-1$. However, his equilibrium does not reduce to ours near the null. Later, Larrabee and Lovelace ${ }^{5}$ examined orbits for particles with $a=-1$ in actual equilibrium fields and observed primarily regular orbits. Orbits of extreme values of have also been investigated. Wang and Miley ${ }^{6}$ studied orbit behavior in FRM where the geometry is characterized by $\varepsilon=1$. Sonnerup ${ }^{7}$ studied the property of $J_{y}$ invariance of particles near a null sheet where $\varepsilon=0$. The present study agrees with these results but goes further by discussing other regions of parameter space as well. Mynick's ${ }^{8-10}$ work on the adiabatic invariance theory for some classes of these particles is extended by discussing other regions of parameter space and by determining the breakdown of adiabatic theory.

The outline of this chapter is as follows. First analytical study based on adiabatic invariant theory is made in Sec. B. We predict some types of regular orbits associated with the invariants and estimate 
the onset of stochasticity. The predictions are supported and refined by numerical observation in Sec. C. The results are summarized in Sec. D. Finally in Sec. E, the result is applied to plasmas of FRC experiments at LANL and the character of ion arbits of the plasma is est imated qualitatively.

\section{B. General Aspects of Particle Motion}

We wish to analyze the motion of charged particles near the null of a two dimensional magnetic field. Specifically, we consider the magnetic field obtained from the vector potential $\underline{A}=\psi(x, y) \hat{z}$, with the flux function $\Psi$ given by

$$
\psi=\frac{B}{2 a}\left(e^{2} x^{2}+y^{2}\right)
$$

where $B_{a}$ is the magnetic field strength at $x=0$ and $y=a$. The magnetic field lines described by the flux function are closed and tangent to the concentric elliptical flux contours shown in Fig. 3.1. On a flux contour, the strength of the magnetic field changes along the ellipse with maximum value at the minor axis and the minimum value at the major axis. For the flux contours away from the null, the magnetic field increases linearly. Such a field represents the magnetic field near the null of a current sheet such as exists in the geomagnetic tail, ribbon-pinch, or a field reversed toroidal system in the limit of large aspect ratio. 
The Lagrangian of a charged particle in such systems is

$$
\frac{1}{2} m x^{2}+\frac{1}{2} m y^{2}+\frac{1}{2} m z^{2}+e z / c
$$

Since the coordinate $z$ is ignorable in this Lagrangian, we immediately deduce that the canonical momentum

$$
P_{z}=\dot{m}+e \psi / c
$$

is a constant of the motion. Use of this invariant allows us to find the effective Lagrangian (or Routhian ${ }^{11}$ ) for the reduced motion:

$$
R=\frac{1}{2} m \dot{x}^{2}+\frac{1}{2} m \dot{y}^{2}-\frac{e^{2}}{2 m c^{2}}\left(\psi(x, y)-\frac{c P_{z}}{e}\right)^{2}
$$

This Lagrangian describes two-dimensional motion in an effective potential

$$
U(x, y)=\frac{e^{2}}{2 m c^{2}}\left(\psi(x, y)-\frac{c P}{e}\right)^{2},
$$

which depends on the value of the invariant $P_{2}$. Notice that the effective potential in $x y p l a n e$ is essentially the kinetic energy of the z-motion.

We further simplify the expression by a scale change. We introduce new variables $X, Y$ and $T$ whose relation to the old is $X=\lambda X$, $y=Y Y$ and $t=T / \Omega$ where $\lambda$ and $\Omega$ are constants. With the definition of dots on the new variables as the derivative with respect to $T$, 


$$
R=m \lambda^{2} \Omega^{2}\left[\frac{1}{2} x^{2}+\frac{1}{2} y^{2}-\frac{1}{2} \frac{e a_{a}^{\lambda}}{2 a m c \Omega}\left(\varepsilon^{2} x^{2}+y^{2}\right)-\frac{P_{z}}{m \lambda \Omega}{ }^{2}\right] .
$$

Since the equations of motion do not depend on the overall constant of $R$ we obtain the equivalent Lagrangian $L$

$$
L \equiv \frac{R}{\Omega^{2}}=\frac{1}{2} \dot{X}^{2}+\frac{1}{2} \dot{y}^{2}-\frac{1}{2}\left(\varepsilon^{2} x^{2}+y^{2}-\sigma\right)^{2}
$$

by choosing

$$
\lambda=\left(\frac{2 a P_{z}}{e_{a}}\right)^{1 / 2}
$$

and

$$
\Omega=\frac{1}{\pi}\left(\frac{{ }_{z}{ }^{e B} a}{2}\right)^{1 / 2}
$$

where $\sigma$ is the sign of $\mathrm{P}_{z} / \mathrm{e} \psi$.

The length is scaled such that the local potential minimum for $0=1$ occurs on the contour of $\varepsilon^{2} x^{2}+y^{2}=1$ and $\Omega$ is the local gyro frequency at $X=0$ and $Y=1$. The scaling breaks down for special particles with $p_{z}=0$, in which case $\sigma=0$ should be taken. However, the motion is obtained as a limit of $H \rightarrow \infty$ in our scaling since the effect of $\sigma$ is negligible in the limit. Therefore we will not study the case of $a=0$ separately.

The motion of a particle can be best analyzed by the constants of motion. The obvious constant of motion of a particle in the time 
independent Hamiltonian system is the Hamiltonian $h$ of the old variable or $H$ of the new variable:

$$
H=\frac{m h}{P_{z}^{2}}=\frac{1}{2} P_{x}^{2}+\frac{1}{2} P_{y}^{2}+\frac{1}{2}\left(\varepsilon^{2} X^{2}+y^{2}-\sigma\right)^{2}
$$

where $P_{X}=\dot{X}$ and $P_{Y}=\dot{Y}$. One more constant af motion will lead the particles to have regular motion, since we already have two exact constants of motion $\mathrm{H}$ and $\mathrm{P}_{Z}$ in three-dimensional system.

The system is integrable (completely regular) for the two extreme values of $\varepsilon$. For the case of $\varepsilon=1$, azimuthal symmetry gives the third invariant $P_{\theta}=\dot{X} Y-\dot{Y} X$, and for the case of $\varepsilon=0$ translational symmetry brings the third invariant $P_{x}$.

The motion for arbitrary $\varepsilon$ values requires detailed study. The effective potential has two different shapes depending on the values of $\sigma$. The effective potential in new variables is

$$
U=\frac{1}{2}\left(\sigma-\varepsilon^{2} x^{2}-Y^{2}\right)^{2}
$$

It is a simple quartic well for $\sigma=-1$ while the quartic potential has a local maximum of value 0.5 at the null for $\sigma=+1$. The range of $H$ is $H>O$ for $O=1$ and $H>0.5$ for $O=-1$. Low energy particles with $H<0.5$ which occurs only for $\sigma=1$ are confined on an elliptical annular ring surrounded by

$$
\sigma-\sqrt{2 H}<\varepsilon^{2} x^{2}+\gamma^{2}<\sigma+\sqrt{2 H}
$$


due to the conservation of $H$. A guiding center motion is expected for the particles whose energy is low enough to be confined in a narrow region around the energy minimum which is also the magnetic field line contour in our case (see plots (a) and (b) in Fig. 3.6). If $H>0.5$, particles are energetically allowed to cross the null (see plots (c) and (d) in Fig. 3.6). The left inequality of Eq. (3.8) is simply neglected and the $X Y$ motion is restricted on a disk. The direction of the magnetic field is opposite across the null. Thus the motion cannot be circularly oscillating but sinusoidal. For very large $H$ values the motion of a particle with $\sigma=1$ would not be too different from that with $\sigma=-1$ since the effect of $\sigma$ is negligible for high $H$.

\section{B.1 Guiding-center Motion}

When the motion of a particle involves two different time scales, it is possible to simplify the motion by averaging the fast motion. The averaging yields an adiabatic invariant associated with it. In guiding center theory ${ }^{12}$ (g.c. theory) the fast time scale is the gyrooscillation time and the slow time scale is the time scale of our interest or other slow motions involved in the motion. It is well known that the invariant associated with the averaging over the gyrooscillation is the magnetic moment defined by $\mu=\frac{W_{\perp}}{B}$ where $W_{\perp}$ is the kinetic energy perpendicular to the magnetic field.

Ordering the Hamiltonian with the adiabaticity parameter, the ratio of the two time scales mentioned earlier, one obtains the Hamiltonian of the guiding center. To the lowest order 


$$
H_{g . c .}=\frac{1}{2 \pi} p^{2}+\mu B(s)
$$

where $P$ and $S$ are the momentum and the distance along the field line and they are canonically conjugate to each other.

We would like to estimate the region in the parameter space of $\varepsilon$ and $H$ in which the g.c. theory is valid. The g.c. theory is likely to be applicable for particles with $H \ll 0.5$ since their motion is confined to a narrow region between the two ellipses as shown in Eq. (3.7). For $\varepsilon \neq 1$, the particles experience weaker magnetic field near the tips of the flux contours. Hence some are trapped at one of the tips magnetically as is the case of particles in mirror machines. For those trapped orbits, the two scalings are the rapid gyro-frequency across the field line and the guiding center bounce frequency $\omega_{B}$ along the field line. If $\omega_{B} / \Omega \ll 1$, the g.c. theory is applicable. The magnitude of the magnetic field at the energy minimum contour, $\varepsilon^{2} X^{2}+Y^{2}=1$, is $2\left(\varepsilon^{4} X^{2}+Y^{2}\right)$ and so smallest at $X=\varepsilon^{-1}$ and $Y=0$. The value is $2 \varepsilon$.

The bounce frequency of the guiding centers can be best obtained by expanding Eq. (3.9) around $s=0$ where $s$ is measured from the major axis. The g.c. Hamiltonian is now

$$
H_{g . c .}=\frac{1}{2 \pi} p^{2}+\mu B(0)+\frac{1}{2} B^{\prime \prime}(0) s^{2},
$$

from which we easily obtain the bounce frequency:

$$
\omega_{B}=\left[H B^{\prime \prime}(0)\right]^{1 / 2}={\frac{W_{1} B^{\prime \prime}(0)}{B(0)}}^{1 / 2}
$$


where $w_{1_{0}}$ is the perpendicular kinetic energy at $s=0$. The condition $\omega_{B} \ll \Omega$ is

$$
W_{10} \ll \frac{4 \varepsilon^{3}}{1-\varepsilon^{2}}
$$

This criterion is expected to be useful for those particles circulating all around the flux contour since they have to experience the magnetic field variation described in Eq. (3.10) every time they pass the magnetic well. Equation (3.11) is a very restrictive condition for small E values. The criterion of small gyro-radius compared to the system size is $W_{1} \ll 1$, which is much less restrictive. The criterion of having well-defined guiding centers, ${ }^{13}\left|\frac{r \cdot \nabla B}{B}\right| \ll 1$, is still $w_{1} \ll \varepsilon^{2}$. Nevertheless, as we will show later, the numerical study shows that the criterion for a g.c. theory to be valid is close to our estimation of $H \ll \varepsilon^{3}$.

Consider a $\mu$ conserving orbit which satisfies the inequality (3.11). From the conservation of $H$ and $\mu$, particles are trapped in one of the magnetic wells if

$$
\frac{W_{10}}{B\left(X=\varepsilon^{-1}, Y=0\right)}>\frac{H}{B(X=0, Y=1)} .
$$

With the relation $B\left(X=\varepsilon^{-1}, Y=0\right)=\varepsilon B(X=0, Y=1)$ and the basic restriction $0 \leq W_{10} \leq H$, the inequality (3.12) becomes

$$
\text { eH } \leq H_{1_{0}} \leq H \text { or } V_{1_{0}}^{2} \geq \frac{\varepsilon}{1-\varepsilon} V_{\|_{0}}^{2} \text {. }
$$


where $v_{10}$ and $v_{\| 0}$ are the velocities perpendicular and parailel to the magnetic field on $Y=0$ plane. The condition for a particle to be circulating all around the flux contour is

$$
0 \leq W_{10} \leq \varepsilon H \text { or } \quad V_{10}^{2} \leq \frac{\varepsilon}{\Gamma-\varepsilon} V_{\| O}^{2} \text {, }
$$

Now we wish to estimate the region of each type of motion on ${ }_{X-P}{ }_{x}$ plane of an energy surface with $Y=0$. (This is the plane that we will use later to obtain surface of section plots numerically). Fig. $3.3(a)$ shows a potential $U(X, Y=0)$ vs. $\varepsilon X$. Since the potential energy is in fact the kinetic energy in $Z, U(X, Y=0)=\frac{1}{2} V_{z 0}^{2}$ where $v_{z 0}$ is the velocity in $Z$ on $Y=0$ plane. For a given $H$, the allowable region on $X-P_{z}$ plane is bounded by the outer contour shown in Fig. 3.3(b). The range on the $x$ axis of Fig. $3.3(b)$ is obtained from the inequality (3.8) by setting $Y=0$ :

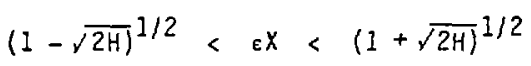

The magnetic field is in $Y$ direction on $Y=0$ plane and so $w_{10}=\frac{1}{2} v_{z 0}^{2}+\frac{1}{2} v_{x 0}^{2}$. Furthermore in the $x$ axis of $F i g .3 .3(b)$ $W_{10}=\frac{1}{2} V_{z 0}^{2}$ since $P_{x}=0$. Therefore the range of $x$ on $P_{x}=0$ axis for a particle to be circulating all around a flux contour is estimated from the inequality $(3.13 a)$ by using a relation $w_{10}\left(P_{x}=0\right)=\frac{1}{2} v_{z 0}^{2}=$ $J(X, Y=0)$ :

$$
\left(1-\sqrt{2 \varepsilon H}^{1 / 2}<\varepsilon X<(1+\sqrt{2 \varepsilon H})^{1 / 2} .\right.
$$


The differences between the ranges in (3.15) and (3.14) is the range of $X$ for trapped orbits. The range of $X$ for trapped orbits is increasing as $\varepsilon$ decreases.

\section{B.2 J Y Conserving Particles}

A generalized constant of motion that is the generalized magnetic moment to the magnetic moment is $\oint \underline{p} \cdot d \underline{q}^{14}$. When $\varepsilon \ll 1$, most particles have much faster $Y$ oscillation than that in $X$, yielding a disparity in $\omega_{X}$ and $\omega_{Y}$ (for example, see Fig. $3.6(c)$ ). In this case

$$
\oint \underline{P} \cdot \underline{d q}=\oint P_{Y} d Y \equiv 2 \pi J_{Y} .
$$

Therefore the action in $Y$ is expected to be an adiabatic invariant. To illustrate the frequency disparity we compare two special motions, one oscillating in $X$ and the other in $Y$. The equation of motion from Eq. (3.6) for the motion along $x$-axis is

$$
\ddot{x}=2 \varepsilon(\varepsilon X)^{3} \text { or }(\ddot{\varepsilon} \dot{X})=2 \varepsilon^{2}(\varepsilon X)^{3} \text {, }
$$

and for the motion along $y$-axis is

$$
\ddot{y}=2 r^{3} \text {. }
$$

Therefore the frequency in $X$ is $\varepsilon$ times slower than that in $Y$; $\omega_{X}=\varepsilon \omega_{Y}$. If $\varepsilon$ is small, a large frequency disparity is expected. 
For such a motion, $X$ and $P_{X}$ can be considered as fixed for a fast oscillation. The adiabatic invariance $J_{Y}$ to the lowest order is then from Eq. (3.16).

$$
J_{Y}=\frac{1}{2 \pi}{ }^{\Phi} P_{Y}\left(H, X, P_{X}\right) d Y \text {. }
$$

Also, the motion in $Y$ is governed by the Hamiltonian

$$
H_{Y}=\frac{1}{2} P_{Y}^{2}+\frac{1}{2}\left(\varepsilon^{2} X^{2}+Y^{2}-\sigma\right)^{2} .
$$

This Hamiltonian describes oscillations in a one-dimensional effective potential $U(Y)=\frac{1}{2}\left(\varepsilon^{2} X^{2}+Y^{2}-\sigma\right)^{2}$ at a fixed value $X$. The potential increases either montonically with $Y$ if $\varepsilon^{2} X^{2}>0$ or has a local maximum of value $U_{0}=\frac{1}{2}\left(\varepsilon^{2} x^{2}-\sigma\right)^{2}$ at $Y=0$ if $\varepsilon^{2} x^{2}<\sigma$. Therefore we define two types of particles. Non-crossing particles are those that cannot energetically cross the $X$-axis with $H_{Y}<U_{0}$ and crossing particles are those that cross the $X$-axis with $H_{Y}>U_{0}$. From the definition of Eq. (3.18)

$$
J_{Y}\left(X, H_{Y}\right)=\frac{1}{\pi} \int_{Y_{1}\left(X, H_{Y}\right)}^{Y_{2}\left(X, H_{Y}\right)} d Y\left\{2 H_{Y}-\left(\varepsilon^{2} X^{2}+Y^{2}-\sigma\right)\right\}_{(3.20)}^{1 / 2}
$$

where the turning points $Y_{1}$ and $Y_{2}$ are obtained from $E q .(3.19)$ by setting $P_{Y}=0$. For axis crossing particles, we simply replace $Y_{1}=0$ in the definition of Eq. (3.20). The definition of $J_{Y}$ for axis crossing particles differs by a factor of two from the definition (3.18), 
which would have the domain of integration be $\left[-Y_{2}, Y_{2}\right]$. The reason is that we want the value $J_{Y}$ to be a continuous function of $H_{Y}$ at $H_{Y}=U_{0}$. With an adiabatic invariant $J_{Y}$ on hand, the motion of particles is understood much better by expressing the motion in terms of the invariant. Then the conjugate coordinate $\varphi_{Y}$ of $J_{Y}$ is ignorable, we obtain the reduced Hamiltonian:

$$
H=\frac{1}{2} P_{X}^{2}+H_{Y}\left(X, J_{Y}\right)
$$

The Hamilton's equations of Eq. (3.21) are

$$
\dot{P}_{X}=\frac{\partial H_{Y}}{\partial X}, \dot{X}=P_{X}, \quad \dot{J}_{Y}=0 \text { and } \dot{\varphi}_{Y}=\frac{\partial H_{Y}}{\partial J_{Y}} \equiv \omega_{Y}
$$

These equations describe the $X$-motion averaged with respect to $Y$ in an effective potential $\mathrm{H}_{Y}$. The contours of $J_{Y}$ are plotted with the $\mathrm{H}_{Y}-X$ plane in Fig. 3.4(a) for $\sigma=-1$ and in Fig. 3.4(b) for $\sigma=+1$.

It is important to know whether the particle stays to one type of motion or not. At the transition point, the frequency,

$$
\omega_{Y}=\frac{\partial H_{Y}}{\partial J_{Y}}=\pi\left[\int_{Y_{1}}^{Y_{2}} d Y\left\{2 H_{Y}-\left(\varepsilon^{2} X^{2}+Y^{2}-0\right)^{2}\right\}^{-1 / 2}\right]_{(3.23)}^{-1 / 2}
$$

vanishes. Hence, adiabatic invariance theory, which is based on $\omega_{Y} \gg \omega_{X}$, cannot be correct near the transition point. Instead we expect $J_{Y}$ to change each time a particle passes through the transition 
point, which causes the particle motion to be stochastic. From the effective potential $H_{Y}(X, Y)$ for a given $X$ it is clear that particles with $a=-1$ or particles with $H>0.5$ for $\sigma=+1$ are axis-crossing. For those with $\sigma=+1$ and $H<0.5$ may experience transition at $X_{c}$ where

$$
H_{Y}\left(X_{C}, J_{Y}\right)=\frac{1}{2}\left(\varepsilon^{2} X_{C}^{2}-\sigma\right)^{2} .
$$

The locus of the transition is shown in $X-H_{Y}$ plane in Fig. 3.4(b). Notice that the curve is below $H_{Y}<0.5$. A particle with $J_{Y}$ whose contour crosses with the transition curve will change its motion from one type to the other at the $X$ values of intersection $x_{c}$. Fig. $3.4(b)$ also shows that no crossing exists if

$$
J_{Y} \geq J_{Y C}\left(H_{Y}=0.5, X=0\right)=\frac{2 \sqrt{2}}{3 \pi} .
$$

Those with $J_{Y}<J_{Y_{C}}$ can change the type of motion depending on whether $H$ allows crossing or not. To illustrate the transition clearly, we show an energy diagram for two different values of $J_{\gamma}$ with $J_{y / 1}>J_{Y 2}$ * For a given value of $J_{Y 1}$ the particle with energy at level $b$ does not experience the transition while the particle with energy level a does. For a given energy value, for example $H=.25$, the particle with action of $\mathrm{J}_{Y_{1}}$ does not experience the transition while the particle with action of $\mathrm{J}_{\mathrm{V}_{2}}$ does. The transition particles are the candidates for the stochastic motion. Since the $x$ coordinate of the potential minimum is always greater than $x_{c}$, non-crossing particles always experience transition, thus are stochastic. Only axis crossing 
u-conserving regular orbits are expected. These cases are tested numerically in Sec. C. (See Fig. 3.10.)

\section{B.3 Summary of Expected Types of Motion}

The expected types of motion for $\varepsilon \ll 1$ are shown in Fig. 3.6-3.8. Fig. 3.6 shows the projected regular motion on $X-Y$ plane. $\mu$-conserving regular orbits are shown in Fig. 3.6(a) and (b), where (a) is the circl lating orbit with $W_{10}: E H$ and $(b)$ is a trapped orbit with $\varepsilon H<W_{1_{0}}<H$ where $H \ll C .5$. Fig. 3.6 (c) and (d) show the $J_{y}$ conserving orbits, both axis crossing. The action values are such that $J_{Y}>J_{Y C}$ for plot (c) and $J_{Y}<H_{Y C}$ for plot (d).

The corresponding $Y 2$ motion is summarized in Fig. 3.7 for regular orbits. The $Y Z$ motion is easily deduced from the two dimensional effecive potential since it is the kinetic energy in $z$ where $\dot{i}=c-r^{2} x^{2}-y^{2}$.

Therefore it is clear that $Z<0$ always for $a=-1$ which is shown in plot (b). When $\sigma=1, i$ changes sign whenever the particle crosses the lux contour of $\varepsilon^{2} x^{2}+y^{2}=1$ which is the potential minimum contour, thus indicates the figure 8 type motion for $H>0.5$ [plot $(c)$ and,$d)]$ and gyro-motion for $H\langle 0.5[p l o t(a)]$. If $\langle\dot{L}\rangle=\sigma-$ $\left\langle E^{2} x^{2}+y^{2}\right\rangle$ is positive the $z$ motion is plot (d) otherwise plot (c). Here the angled bracket is the time average over one period of the fast oscillation. For large $H$ values we expect the motion to be similar to that of $\sigma=-1$ since the effect of $\sigma$ is negligible for large $H$. Therefore, we exsect the $Y Z$ motion of large $H$ values to be plot (c). 
When the particle changes its motion from one type to the other, its motion tends to be stochastic. An example of a stochastic motion is shown in Fig. 3.8(a). Also a fixed point orbit and a resonance orbit are shown in Fig. 3.8 (b) and (c) respectively.

\section{Numerical Analysis}

In this section we present numerical observations to verify and sharpen the inequalities of ihe last section and to certify that no other types of motion exist. The surface of section plots ${ }^{15}$ are used to determine the existence of an invariant. Actual motions projected onto the $X Y$ plane along with the adiabatic invariant vs. time are also illustrated for typical types of orbits.

\section{C.1 Variation with $H$ at $\varepsilon=0.2$}

A series of surface of section (SOS) plots are shown in Fig. 3.9 each corresponding to different $H$ values at fixed $\varepsilon=0.2$. The SOS plots are obtained in the usual way. The initial conditions can be chosen arbitrarily on an energy surface. We chose $Y(0)=0, F_{X}=0$ and $X(0)$ in the allowable region $\frac{1}{2}\left(\varepsilon^{2} X^{2}-a\right)^{2} \leq H$, and $P_{Y}(0)=$ $\left[2 H-\left(\varepsilon^{2}(x(0))^{2}-a\right)^{2}\right]^{1 / 2}$. Then we follow each particle by integrating the equations of motion. Every time the particle passes through the $Y=0$ plane with $P_{Y}>0$, the value of $X$ and $P_{X}$ are plotted. We follow the particle for an arbitrary long time until we have enough dots on the $X-P_{X} p l a n e$. The solid line on the figures is the energy boundary. Only the region $x>0$ is plotted since it is symmetric in $x$. 
The first two plots of Fig. 3.9 are the case of $\sigma=-1$. Plot (a) is for $H=0.5$ and plot (b) is for $H=.55$. Six initial conditions are taken for plot (a) and five for plot (b). The SOS plot of each initial condition lies on smooth curves indicating their regular behavior. As will be shown later, the third invariant in the case of $\varepsilon \ll 1$ is $J_{Y}$.

The remaining six SOS plots fig. $3.9(c)-(h)$ are for $\sigma=+1$. In this case the SOS $p^{\top}$ ot is simiiar to that of $\sigma=-1$, which is expected, since the effect of $\sigma$ is negligible for large $H$. As $H$ is reduced to 1 (near 0.5 ) as in Fig. 3.9(d), some stochastic behavior appears in the outer region of the energy surface. In addition, a small island is observed at $P_{X}=0$. The motion corresponding to that island is shown in Fig. $3.10(b)$, the end trapped $J_{Y}$ conserving orbit. The reason that a particle is trapped, and not covering the whole energy-allowable region, is due to the invariance of $J_{Y}$ just as the $\mu$-conservation does to particles in a magnetic mirror.

As $H$ decreases to 0.5 more particles are stochastic except onty a small portion of end trapped $J_{\gamma}$ conserving orbits. It is an interesting phenomenon that the centers of the $J_{Y}$ invariant curves of plots (d) and (e) do not match to the energy minimum position. Furthermore the center of the invariant curves are close to the $X=0$ plane for high $H$ values as in plot (d). This phenomenon is easily understood from fig. 3.5. From the figure we know that the minimum of the one-dimensiona? potential is always nearer to the $X=0$ plane than that of the twodimensional case. The $x$-position of the one-dimensional potential minimum is closer to the $X=0$ plane as $J_{Y}$ (or $H$ ) increases. 
When $H=0.05$, as in Fig. 3.9 (f), nearly all of the phase space region is stochastic. This persists down to very low $\mathrm{H}$ values as shown in Fig. $3.9(\mathrm{~g})$. For $H$ values as low as $1.2 \times 10^{-4}$, $\mu$-conserving g.c. motion appears. Outer invariant curve of plot (g) is the magnetic trapped orbit. The circulating regular orbit has not appeared yet. Finally, at very small values of the energy (see plot (h)) for $H=5 \times 10^{-5}$ primarily regular behavior is again seen throughout phase space. Outer invariant curves are from the magnetically trapped orbits and the inner curves are from the circulating orbits. The stochastic region between the two regular regions is not noticeable. The region of each regular motion is obtained from fig. 3.3. The transition between plots (f) and (h) allows us to refine the inequality (3.11) to

$$
H \leqslant .04 \varepsilon^{3}
$$

for guiding-center motion to be applicable.

\section{C.2 Observed Trajectories for $\epsilon=0.2$}

Sample trajectories of the types of motion in the surface of sections of Fig. 3.9 along with the values of proposed invariants are shown in Fig. 3.10-12.

Fig. 3.10(a) shows the trajectory of a particle with $\sigma=1$ and $H=5$, corresponding to $F i g .3 .9(\mathrm{c})$. The right half of the $X Y$ trajectory, shown in Fig. 3.10(a), illustrates the frequency disparity in $x$ and $Y$ oscillations. Thus, Fig. 3.10(b), which shows good conservation 
of the action $J_{Y}$ calculated from Eq. (3.20) should not be too surprising. If one evaluates $J_{Y}$ exactly using exact $X$ and $P_{X}$ values, the fluctuations of $J_{Y}$ are reduced. Notice that the action quantity of plot (b) satisfies the inequality $J_{Y}>J_{Y C}$ and the motion is axiscrossing all the time. For $\sigma=-1$, the same behavior is observed as far as $J_{Y}$ conservation and the $X Y$ projected motion go. The only difference is the Z-motion. The Z-motion of $\sigma=-1$ is betatron while that of $\sigma=+1$ is figure 8 type.

Fig. 3.10(b) shows the trajectory of a $J_{Y}$ trapped particle for the case $\sigma=1$ and $H=0.25$. The $X Y$ trajectory [Fig. 1O(a)] again illustrates the fact that the $Y$-frequency is much greater than the $x$-frequency, which leads $J_{Y}$ to be a good invariant $[p \operatorname{lot}(d)]$. The $J_{Y}$ value of plot (d) is -0.2 which is less than the critical value $J_{Y C}$. To see whether our analysis did predict a regular motion for $J_{Y}=0.2$ with $H=.25$ we go back to Fig. 3.5. The curve $J_{Y}=0.2$ corresponds to the upper curve of the figure and $H=.25$ is the level b. It shows that the particle is not transitioning but remains to be axis crossing, thus showing the regular behavior consistent with the numerically observed motion.

Fig. 3.11 shows the motion of $\mu$-conserving orbits. Two trajectories of $H=5 \times 10^{-5}$ are considered; one for a magnetically trapped [plots (a)(b) and (c)] and the other circulating regular motion [plots $(d)(e)$ and $(f)]$. The peak of the fluctuation in $\mu$ of plots (b) and (e) occurs when particles pass through the magnetic well. This phenomenon is common for a trajectory in a magnetic mirror. We computed 
$\mu$ by using the local magnetic field. The fluctuation of $\mu$ between the peaks is reduced if one adds the higher order corrections to $u$. The small magnetic jumps in plot (e) do not imply the lack of an invariant, and so $\mu$ can be still considered as a good adiabatic invariant. Plots (c) and (f) show the periodic motion. They also show whether the motion is confined to one end of flux contours or circulating. It is interesting that the X-motion shown in plot $(f)$ is like a free particle motion bouncing back and forth at some $x$.

Finally we show a stochastic motion in Fig. 3.12. To show where the jumps in $\mu$ and $J_{Y}$ occur, we evaluated the values with respect to $X$ [plots (b) and (c)]. The jump values occur at the magnetic well.15 Especially the jumps in $J_{Y}$ occur when the motion changes from axiscrossing to non-axis crossing or vice versa. Plot (d) shows the aperiodic motion and plot (e) and (f) show how wildly the values change in time, indicating the lack of the third invariant.

\section{C.3 Scaling with $E$ at fixed $H$}

To illustrate how the orbit behavior changes as $\varepsilon$ varies, we present one more series of SOS plots in Figs. 3.13 and 3.14. Fig. 3.13 shows the SOS plots for $H=5, \sigma=1$ in decreasing order of $c$. The $\varepsilon$ values are $1.0,0.9,0.5$ and 0.2 from plot (a) to (d). The best way to understand the figure is to compare the two limiting cases of $\varepsilon$ values Fig. 3.13(a) and (d). Both show very smooth invariant contaurs but no contours cross the $x=0$ axis in Fig. 3.13(a) while all the contours cross the $X=0$ axis in Fig. $3.13(d)$. It is because $P_{\theta}$ is 
a constant of motion when $\varepsilon=1$ and thus the centrifugal potential $p_{0}^{2} / 2 r^{2}$ do not allow particles to pass through the origin except very special particles with $P_{\theta}=0$. It is $J_{Y}$ that is conserved for $\varepsilon \ll 1$ and not $P_{\theta^{*}}$

The transition between these $\varepsilon$ values is shown in plots (b) and (c). In plot (b) $(\varepsilon=.9)$ half the trajectories are similar to the $P_{\theta}$-conserving trajectories of the case $\varepsilon=1$. The other half are similar to the $J_{Y}$-conserving trajectories of the case $E=0.2$. At $\varepsilon=0.5$ the $F_{\theta}$-conserving trajectories are destroyed. A large stochastic region is observed. At $\epsilon=0.5$ a large resonance structure of $\omega_{Y} / \omega_{X}=2$ is usually seen for $H>0.5$.

We do not present the SOS plots for $\sigma=-1$ for various $\varepsilon$ values. The plots are qualitatively the same as Fig. 3.13 that we have just studied. The oniy difference is that the resonance structure of $\varepsilon=0.5$ does not bring apparent ergodic behavior.

Fig. 3.14 shows the SOS plots for $H=.005$ again with the same sequence of $\varepsilon$ values as in Fig. 3.13. When $\varepsilon \geq 0.5$ the inequality (3.25) is satisfied and orbits are observed to be integrable with g.c. motion. For $\epsilon=0.5$ there appear two types of $\mu$-conserving orbits, one is the trapping orbit shown as an invariant curve near the energy boundary in Fig. 3.14(c) and the other is the circulating orbit shown as invariant curves near the center in the same SOS plot. Orbits that experience both trapping and circulating motions are stochastic. They fill up the energy surface between the two regular regimes of trapping and circulating. Here the values of $\mu$ of the stochastic motion 
diffuses slowly, filling up the energy surface rather slowly. When $3=0.2$, the inequality (3.25) is violated and thws shows stochasticity of the orbits.

D. Sumary of Orbit Analysis

The analytical methods of Sec. B and the numerical methods of Sec. C allow us to present a broad overview of particle motion near a linear magnetic nul1. These results are conveniently expressed in terms of $\varepsilon$ and $H$ for a given sign of $\sigma$. (1) For $\sigma=-1$, most orbits are regular with betatron type motion, i.e. the velocity in the axial direction does not change. When $\varepsilon \simeq 1$, the canonical angular momentum associated to the azimuthal symmetry is an invariant. When $\varepsilon \ll 1$, the action in the minor radius dimension associated witn the disparity of the frequencies in the major and the minor dimension is a constant of motion. (2) For $a=+1$, a rich behavior of orbits is observed, which can be categorized roughly into three groups. The results are shown in Fig. 3.15. The dots are drawn where the SOS plots are obtained, (a) For $H \gg 0.5$ the behavior of orbits are similar to that of $0=-1$ except the axial motion is figure eight type rather than betatron. This is because the effect of $a$ is negligible for high $H$ values. The motion is smoothly changing from $\sigma=+1$ to that of $\sigma=-1$ in the limit of $H \rightarrow$. (b) For very small energy values $H<.04 \varepsilon^{3}$ the orbits are regular with the third invariant being the magnetic moment of guidingcenter theory. (c) For the intermediate regime $.04 \varepsilon^{3}<H<0.5$ most orbits are stochastic. The stochasticity comes from the random jumps 
in values of $\mu$ or $J_{y}$ whenever the particles pass the weak magnetic field region around the tip of the flux surface.

\section{E. Application to Experiments}

We apply our results to characterize the ion (more precisely deuteron) motion in FRC experiments at LANL. In fact, no exact conparison can be made since the experiments are toroidal systems. Two examples will be considered for comparison: FRX $B$ and FRX-C, in which the major difference is the size oi the plasma. As a typical experimental parameter, we choose for a FRX-B the minor separatrix radius $a=2 \mathrm{~cm}, \varepsilon=0.1$ and $T_{j}=200 \mathrm{eV}$. The magnetic field at $x=0$ and $y=a$ which is denoted as $B_{a}$, in the text, is $6 \mathrm{KG}$. For a FRX-C we choose $\mathrm{a}=4 \mathrm{~cm}$ and keep the rest of the parameters the same as those of FRX-B.

In order to study the character of orbits of a given spatial position, we need to express the result in terms of $P_{z}$ explicitly. This can be done easily since the sign of $\sigma$ can be defined to be the sign of $P_{z}$ and $H=m h / P_{z}^{2}$ where $h$ is the Hamiltonian or the energy of a particle. For the particles with $P_{z}<0,2 \mathrm{mh}>p_{z}^{2}$ should hold and the orbits are regular, $J_{Y}$ conserving. For the particles with $P_{Z}>0$, the motion is regular $J_{Y}$ conserving motion for $2 m h \gg P_{Z}^{2}$, regular g.c. motion for $2 m h<.04 \varepsilon^{3} P_{z}^{2}$ and otherwise stochastic. These regimes are plotted in Fig. 3.16(a) in the parameter space of $\sqrt{2 \mathrm{mh}}$ and $P_{z}$. The slope of transition 1 ines are 1 and $0.2 \varepsilon^{3 / 2}$. Particles with many different $h$ and $P_{z}$ values can pass through a given point $(x, y)$. Depending on the particle position, on? certain regions of $h$ 
and $P_{z}$ are accessible. The restriction comes from the fact that the kinetic energy of a particle should not be negative at any position; i.e., $h \geq \frac{1}{2 m}\left(P_{z}-\frac{e}{c}\right)^{2}$. Furthermore if we assume that the velocity distribution is roughly Maxwellian with temperature T, most particles satisfy $h \leq \frac{3}{2} T$. The accessible region of $h-P_{z}$ space for a particle to appear at a given position $(x, y)$ is shown in $F i g .3 .16(b)$. The allowable region is bounded by a triangle with the bottom vertex at

$$
P==\frac{e B a}{2 c a}\left(e^{2} x^{2}+y^{2}\right)
$$

The triangle moves to the right from the origin as the position of our interest moves away from the null.

To characterize the orbits that pass through a given configuration space $x$ and $y$, we simply overlap Fig. 3.16(b) to (a). By overlapping the plot (b) to (a) we see that all particles are regular, $J_{y}$ - conserving at the null. A little away from the null stochastic orbits appear.

If the plot (b) is moved to the right so that the bottom vertex of the accessible region passes $P_{z}=\sqrt{3 m T}$ most orbits are stochastic. To see mostly u-conserving orbits, plot (b) should be moved to the right until the inequality $P_{z}>3 m T / 2 \varepsilon^{3 / 2}$ is satisfied. With Eq. (3.26) the conditions are

$$
\frac{y_{j Y}}{a}=\left(\frac{2 c \sqrt{3 m T}}{a_{a} B_{a}}\right)^{1 / 2}=1.8\left(\frac{\sqrt{T(100 e v)}}{a c(m) B_{a}(K G)}\right)^{1 / 2}
$$


for the transition from $\mathrm{J}_{Y}$ conserving to stochastic motion, and

$$
\frac{y_{\text {g.c. }}}{\bar{a}}=\left(0.2 e^{3 / 2}\right)^{-1 / 2} \frac{y_{J Y}}{a}
$$

for the transition from stochastic to $u$-conserving where $y_{\mathrm{JY}}$ and

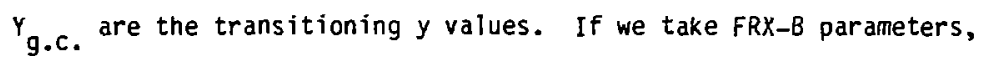

$$
\frac{y_{\mathrm{JY}}}{\mathrm{a}}=.6 \quad \text { and } \quad \frac{y_{\mathrm{g} \cdot \mathrm{c} .}}{\mathrm{a}}=7.2 \text {. }
$$

For FRX-C, both values are reduced by $\sqrt{2}$ due to the size difference. Therefore ions of the FRX experiments at LANL are mostly regular near the null and mostly stochastic near the boundary. Essentially no orbits obey g.c. dynamics.

If we consider a confined plasma in which no bounce orbits at the plasma edge are allowed, an additional restriction is

$$
\sqrt{2 m h}<\frac{1}{2}\left(P_{2}-\frac{e \psi_{B}}{C}\right)
$$

where $\psi_{B}$ is the flux quantity at the plasma edge. The overall behavior of orbits of a confined plasma can be seen from Fig. 3.16(c), from which we see that as the temperature of particles increases more orbits are regular (both for FRX-B and FRX-C) and as the size of the plasma increases more orbits are stochastic (from $F R X-B$ to $F R X-C$ ). 
REFERENCES AND FOOTNOTES

1. A. G. Es'Kov, K. Ku Kurtmallaev, A. P. Kreschuk, Ya N. Lankin, A. I. Molyutin, A. I. Markin, Ya S. Martyuskov, B. K. Mirov, M. M. Orlov, A. P. Proxletsov, and Yu B. Sosunov, in Plasma Physics and Controlled Nuclear Fusion Research (IAEA, Vienna, 1978), Vol. II, p. 187.

2 W. T. Armstrong, R. K. Linford, J. Lipson, D. A. Platts and E. G. Sherwood, Phys. Fluids 24, 2068 (1981).

3. K. F. Mckenna, W. T. Armstrong, R. R. Bartsch, R. E. Chrien, J. C. Cochrane, Jr., R. W. Klewish, Jr., P. Klingner, R. K. Linford, D. J. Rej, E. G. Sherwood, R. E. Siemon, and M. Tuszewski, Phys. Rev. Lett. 501787 (1983).

4. J. M. Finn, Plasma Phys. 21, 405 (1979).

5. D. A. Larrabee and R. V. Lovelace, Phys. Fluids 23, 1436 (1982).

6. M. Y. Wang and G. H. Miley, Nucl. Fusion $\underline{19}, 39$ (1979).

7. B. U. O. Sonnerup, J. Geophys. Res. 768211 (1971).

8. H. E. Mynick, Phys. Rev. Lett. 43, 1019 (1979).

9. H. E. Mynick, Phys. Fluids 23, 1888 (1980).

10. H. E. Mynick, Phys. Fluids 23, 1898 (1980).

11. H. Goldstein, Classical Mechanics (Addison-Wesley, Reading, MA, 1950), p. 218.

12. T. G. Northrop, The Adiabatic Motion of Charged Particles (Interscience, New York, 1963). 
13. G. Schmidt, Physics of High Temperature Plasmas (Academic, New York, 1979), p. 10.

14. V. I. Arnold, Mathematical Methods of Classical Mechanics (Springer-Verlag, New York, 1978) p. 238.

15. V. I. Arnold and A. Avez, Ergodic Problems of Classical Mechanics (W. A. Benjamin, New York, 1968), p. 230.

16. R. J. Hastie, G. D. Hujiss, and J. B. Taylor, in Plasma Physics and Controlled Nuclear Fusion Research (IAEA, 1969) vol. 1, p. 389. 


\section{FIGURE CAPTIONS}

Fig. 3.1 Contours of the flux function and the magnetic field lines for arbitrary $\varepsilon$.

Fig. 3.2 Effective potential $U(X, Y)$ for $\sigma=\neq 1$.

Fig. 3.3 An effective potential $U(X, Y=0)$ and a diagram showing the regions in $X-P_{X}$ space of circulating orbits and trapped orbits. Drawn in plot (a) is a diagram of an effective potential $U\left(X, Y{ }_{Z O}\right) . U(X, Y=0)$ is in fact the kinetic energy of a particle with $P_{X}=0$ on $Y=0$ plane perpendicular to the magnetic field, $W_{10}\left(P_{X}=0\right)$. Plot (b) shows the regions of $X-P_{X}$ space of $Y=0$, the circulating and the magnetically trapped particles of energy $\mathrm{H}$. From inequality (3.13), inside of the inner curve is for circulating orbits and the annular region is for trapped particles of energy $H$.

Fig. 3.4 The effective potentials $H_{Y}\left(x, J_{Y}\right)$ for various $J_{Y}$. Plot (a) is for $\sigma=-1$ and plot (b) is for $\sigma=1$. Values of $H_{Y}$ ard $X$ below the bottom solid line are unphysical in both cases. The locus of transition points discussed in the text is shown in plot $(b) . J_{Y c}$ is the critical value of $J_{Y}$.

Fig. 3.5 Energy diagram of $J_{Y}<J_{Y C}$. Solid curves are effective potentials $H_{Y}\left(X, J_{Y}\right)$ for two different $J_{Y}$ values with $J_{Y_{2}}<J_{Y_{1}}$. The dashed curve is the locus of transition points. 
Fig. 3.6 XY trajectories of regular motion. ${ }^{\prime}$-conserving orbits are in (a) and ( 0 ) and $J_{Y}$-conserving orbits are in (c) and (d).

Fig. 3.7 $Y Z$ trajectories of regular motion.

Fig. 3.8 $X Y$ trajectories of stochastic and resonance motion. Shown are the $X Y$ trajectories of a stochastic orbit (a), a fixed point orbit of $\omega_{X} / \omega_{Y}=5(b)$ and a resonance orbit of $\omega_{X} / \omega_{Y}=5(c)$.

Fig. 3.9 Surface of section plots of $\varepsilon=.1$. Plots (a) and (b) are for $a=-1 . H=5$ in (a) and $H=.55$ in (b). Plots (c)-(h) are for $\sigma=I$ in decreasing order in $H . H=5(c)$, $H=1(d), H=.5(e), H=5 \times 10^{-3}(f), H=1.2 \times 10^{-4}(g)$ and $H=5 \times 10^{-5}(h)$.

Fig. 3.10 Trajectories for $J_{Y}$-conserving motion for the case $\varepsilon=.2$ and $\sigma=1$. Plots $(a)$ and (b) are for $Y$-axis-crossing particle of $H=5$. Initial conditions are $X(0)=5.0$, $Y(0)=P_{X}(0)=0$ and $P_{Y}(0)$ calculated from $H$. Plots (c) and (d) are for non-Y-axis-crossing particle of $H=.25$. Initial conditions are $X(0)=3.14 Y(0)=$ $P_{X}(0)=0$ and $P_{Y}(0)$ calculated from $H$. The $J_{Y}$ values in (c) and (d) are evaluated by using Eq. (3.20).

Fig. 3.11 Trajectories for $u$-conserving motion for the case $\varepsilon=0.2$, $\sigma=1$ and $H=5 \times 10^{-5}$. A magnetically trapped motion is shown in (a), (b) and (c) and a circulating motion is in (d), (e) and (f). The initial conditions of the trapped orbit are $X(0)=5.01, Y(0)=P_{X}(0)=0$ and $P_{Y}(0)$ is calculated from $H$. The initial conditions of the 
circulating orbit are chosen similarly with $x(0)=4.98$. The magnetic moments shown in (b) and (e) are evaluated by using local magnetic fields.

Fig. 3.12 Trajectories of a stochastic motion for the case of $\varepsilon=0.2, \sigma=1$ and $H=5 \times 10^{-3}$. Initial conditions are $X(0)=4.87, Y(0)=P_{X}(0)=0$, and $P_{Y}(0)$ is calculated from $H$. Shown are the $X Y$ trajectory $(a), J_{Y}$ vs $X(b), \mu$ vs $X(c), X$ vs $T(d), J_{Y}$ is evaluated by Eq. (3.20) and $\mu$ is evaluated by using local magnetic fields.

Fig. 3.13 Surface of section plots for $H=5$ and $\sigma=1$ : in (a) $\varepsilon=1$; in (b) $E=0.9$; in $(c) E=0.5$; in (d) $E=0.2$.

Fig. 3.14 Surface of section plots for $H=5 \times 10^{-3}$ and $\sigma=1$ : in (a) $E=1$; in (b) $\varepsilon=0.9$; in (c) $E=0.5$; in (d) $\varepsilon=0.2$.

Fig. 3.15 Diagram out lining stochastic and regular regions of parameter space for $\sigma=+1$. Surface of section analyses were done at the parameter values of the dots.

Fig. 3.16 Regions of parameter space and the allowable regions of parameter space of confined plasmas. Plot (a) shows the types of motion occurring in various regions of parameter space. The slope of solid lines are \pm 1 and the slope of the dashed line is $\varepsilon^{3 / 2}$. Plot (b) shows the allowed range of parameters for a Maxwellian of temperature $T$ at a position $\left(x_{0}, y_{0}\right)$ where $P_{z 0}=\frac{e B_{a}}{2 c a} \phi_{0}$ with $\phi_{0}$ being the value of $\phi=\varepsilon^{2} x^{2}+y^{2}$ at $x=x_{0}$ and $y=y_{0}$. Plot (c) shows the allowable regions in the parameter space of plasmas in FRX-B and FRX-C at LAilL. 


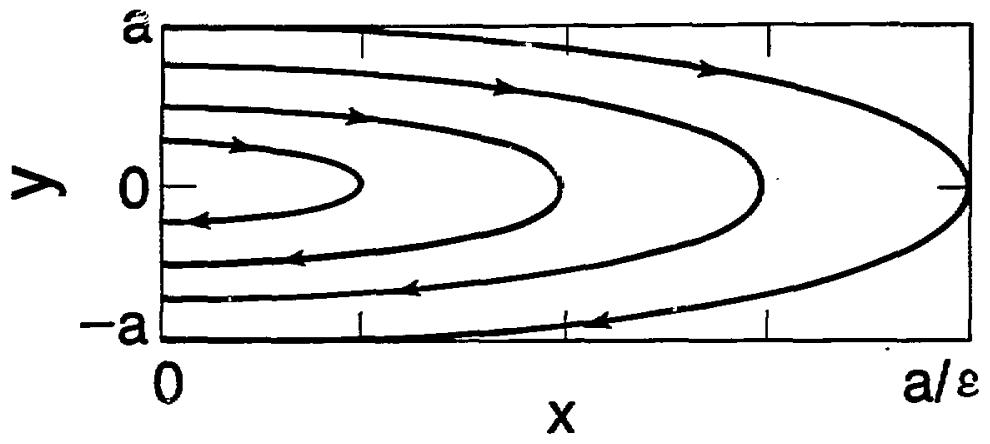

Figure 3.1

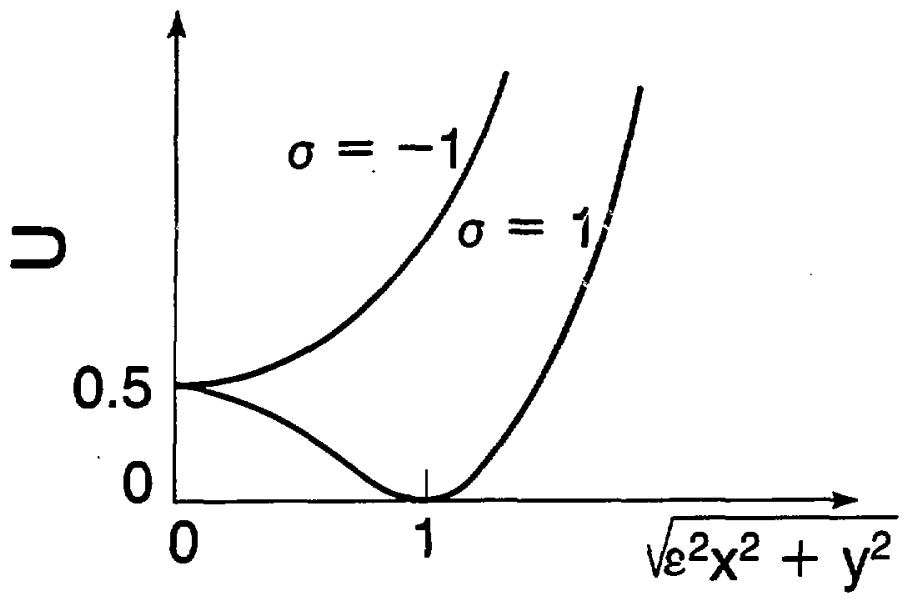

Figure 3.2 


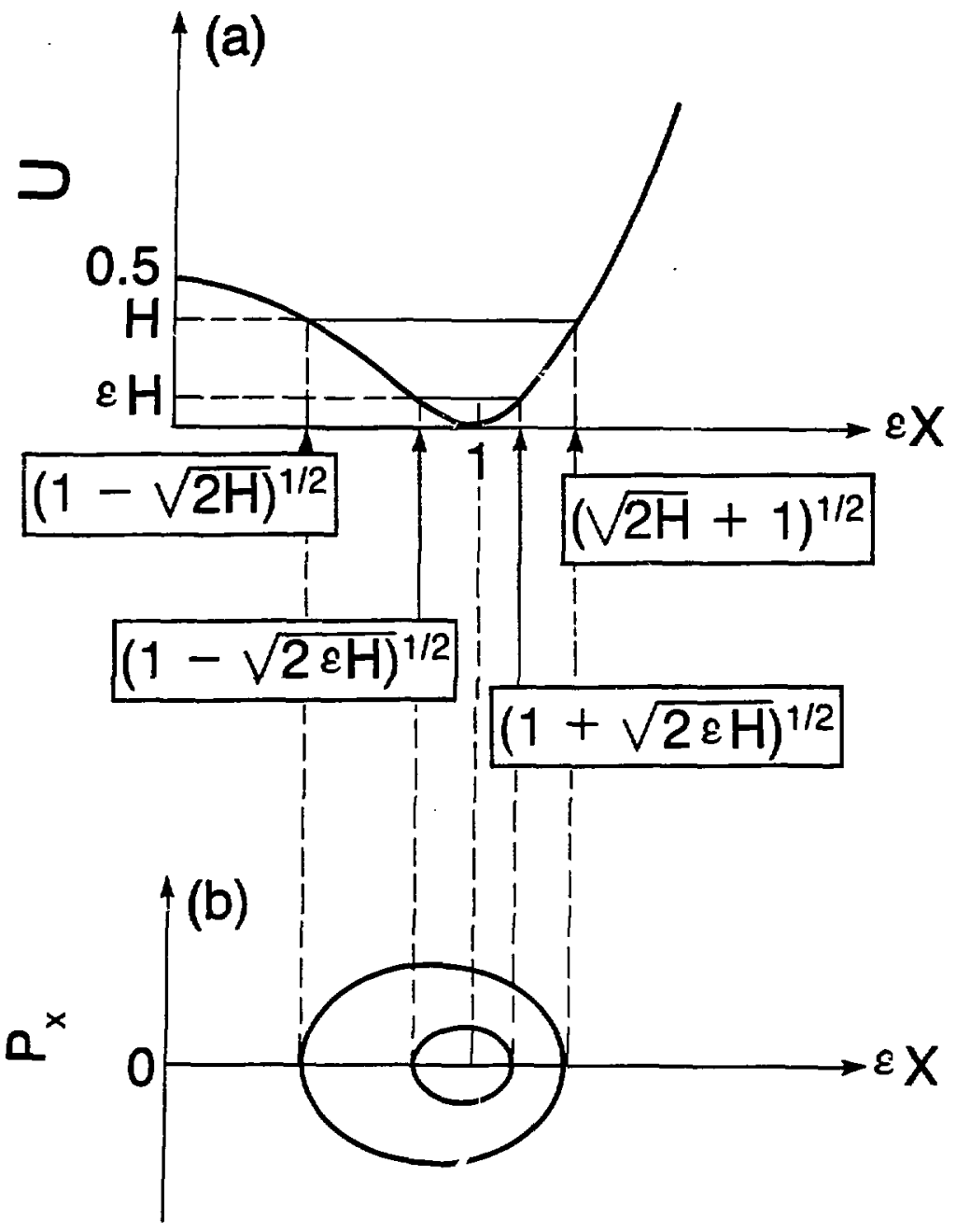

Figure 3.3

XBL 83:0-3338 

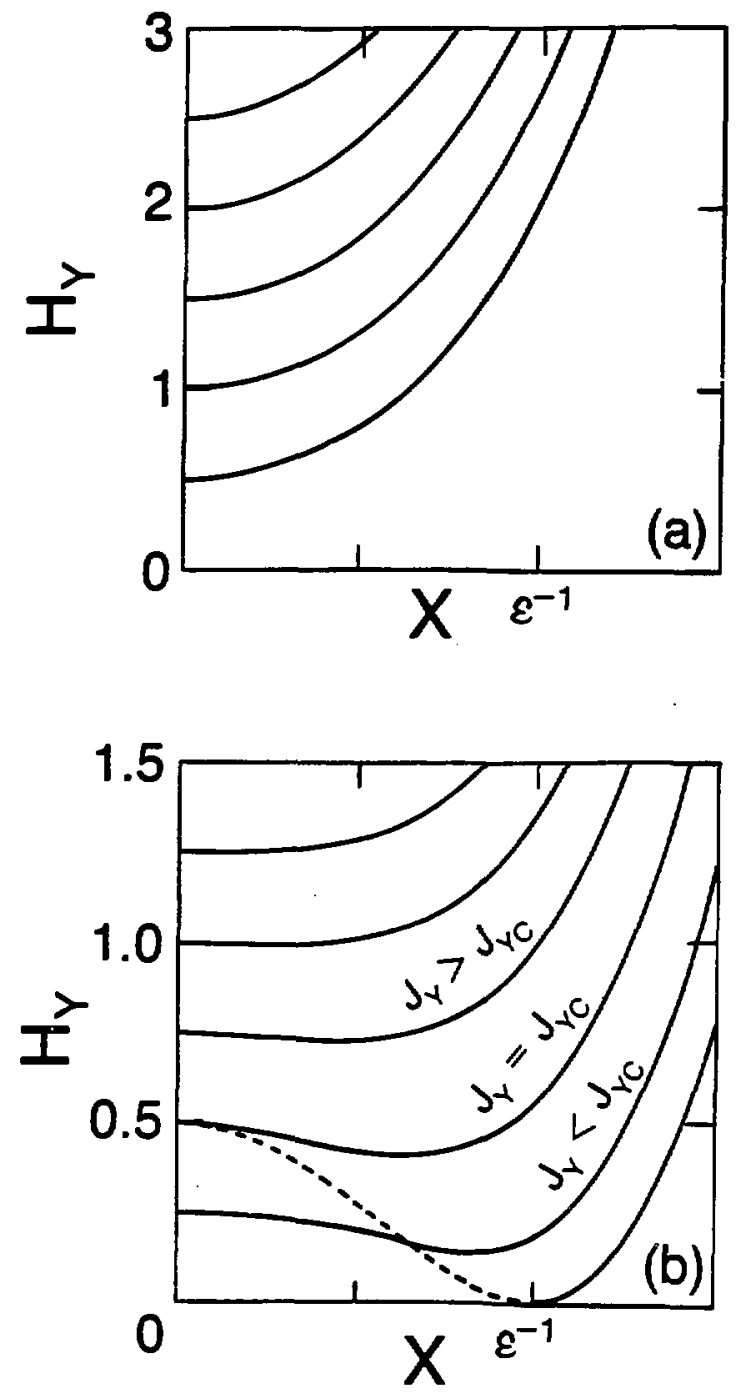

Figure 3.4 


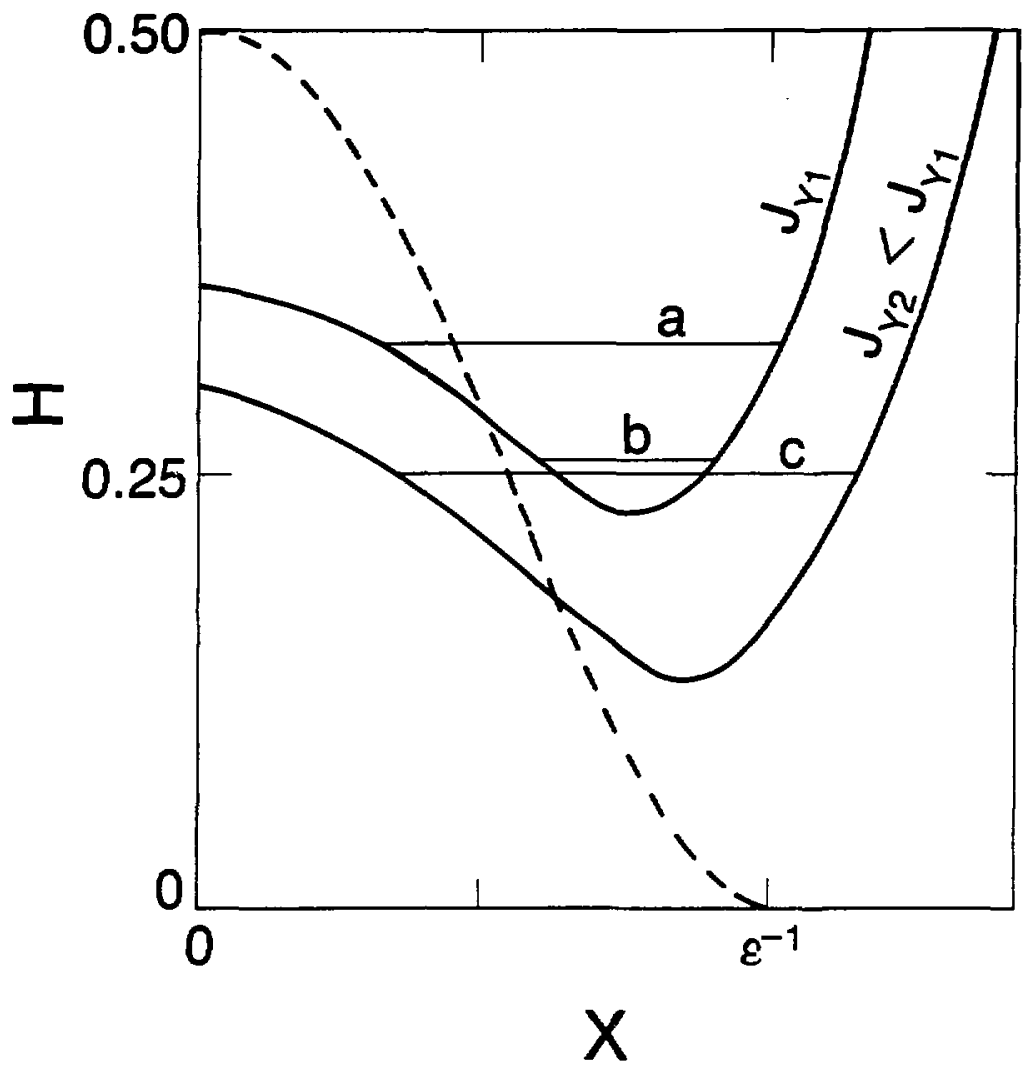

XBL 8310-3328

Figure 3.5 

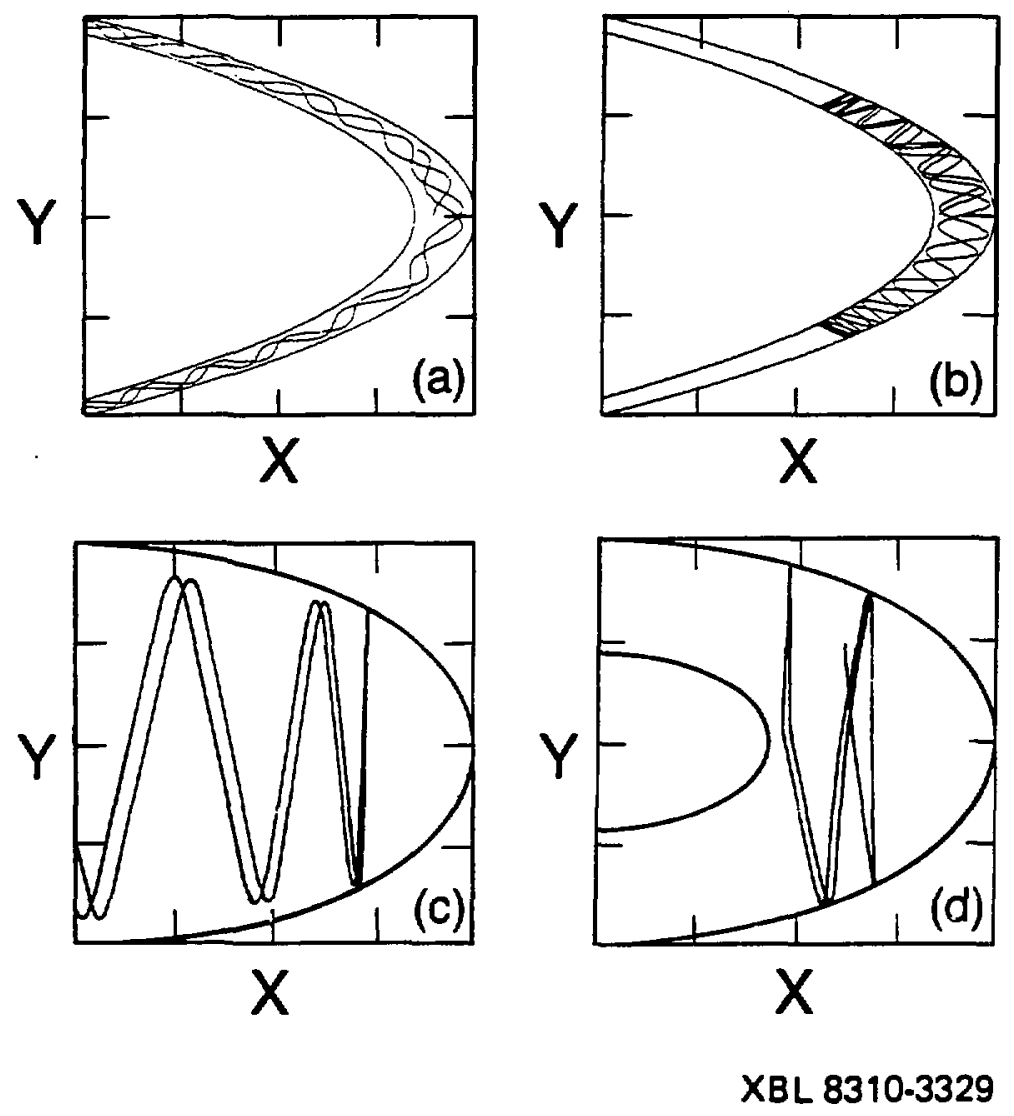

Figure 3.6 

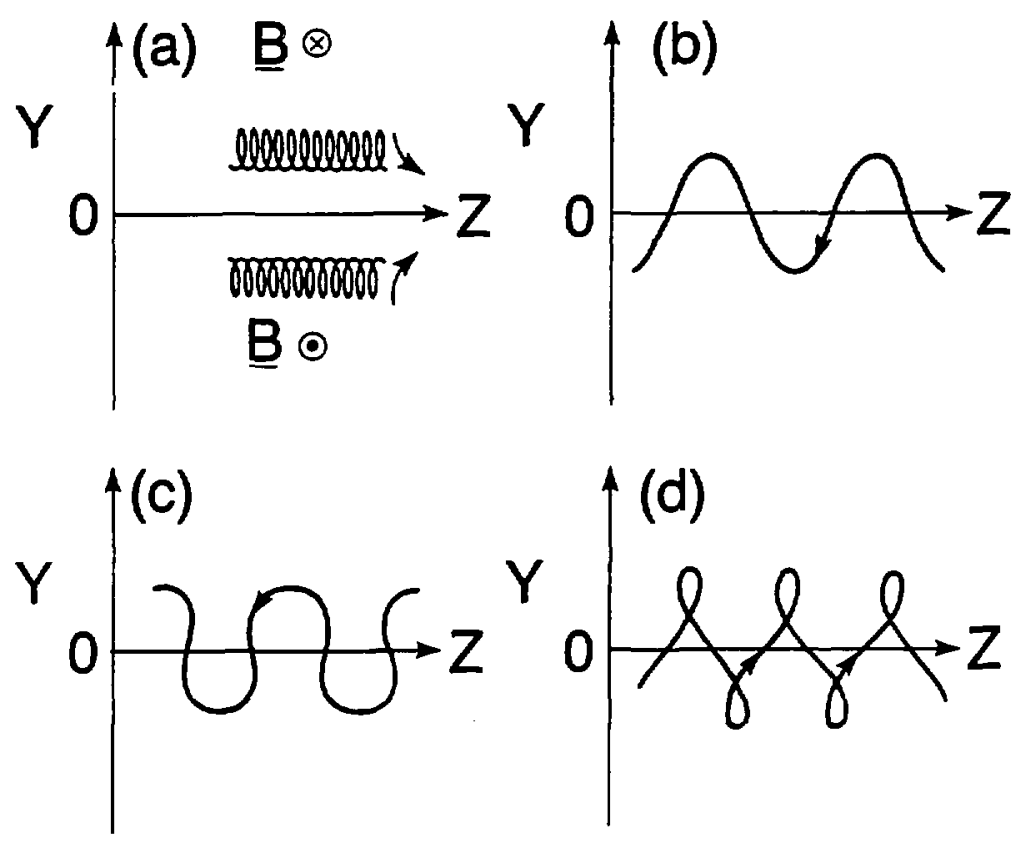

XBL $8370-3334$

Figure 3.7 

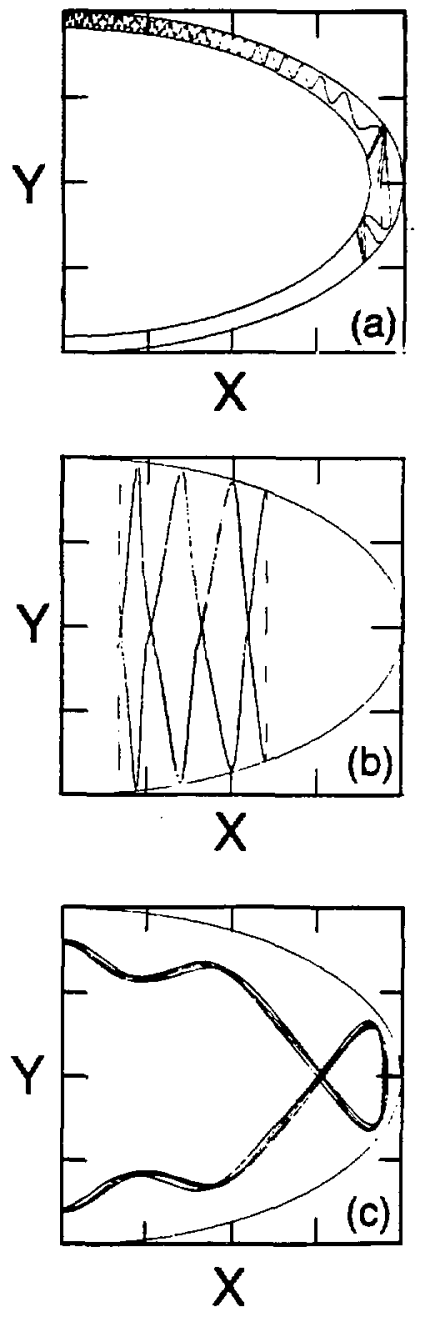

Figure $3.8 \quad$ XBL $8310-3333$ 

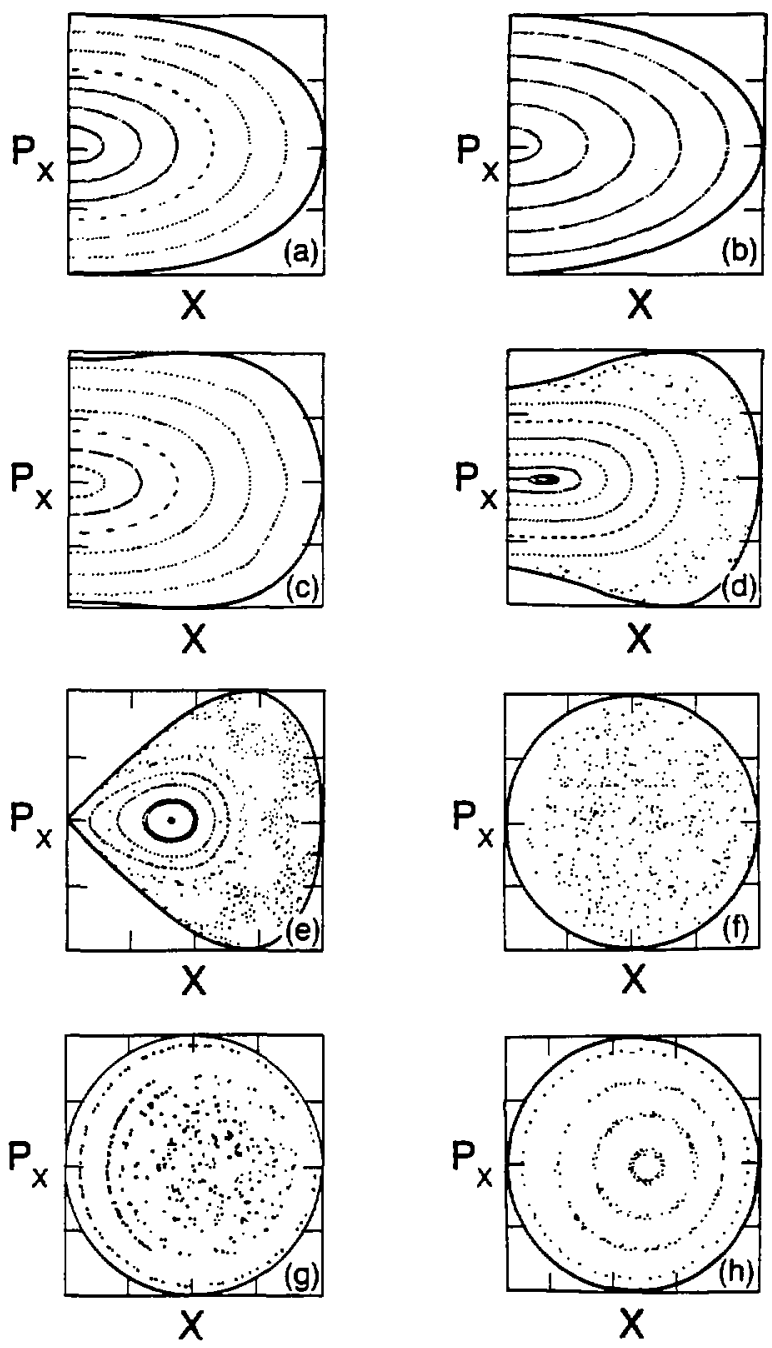

Figure 3.9 

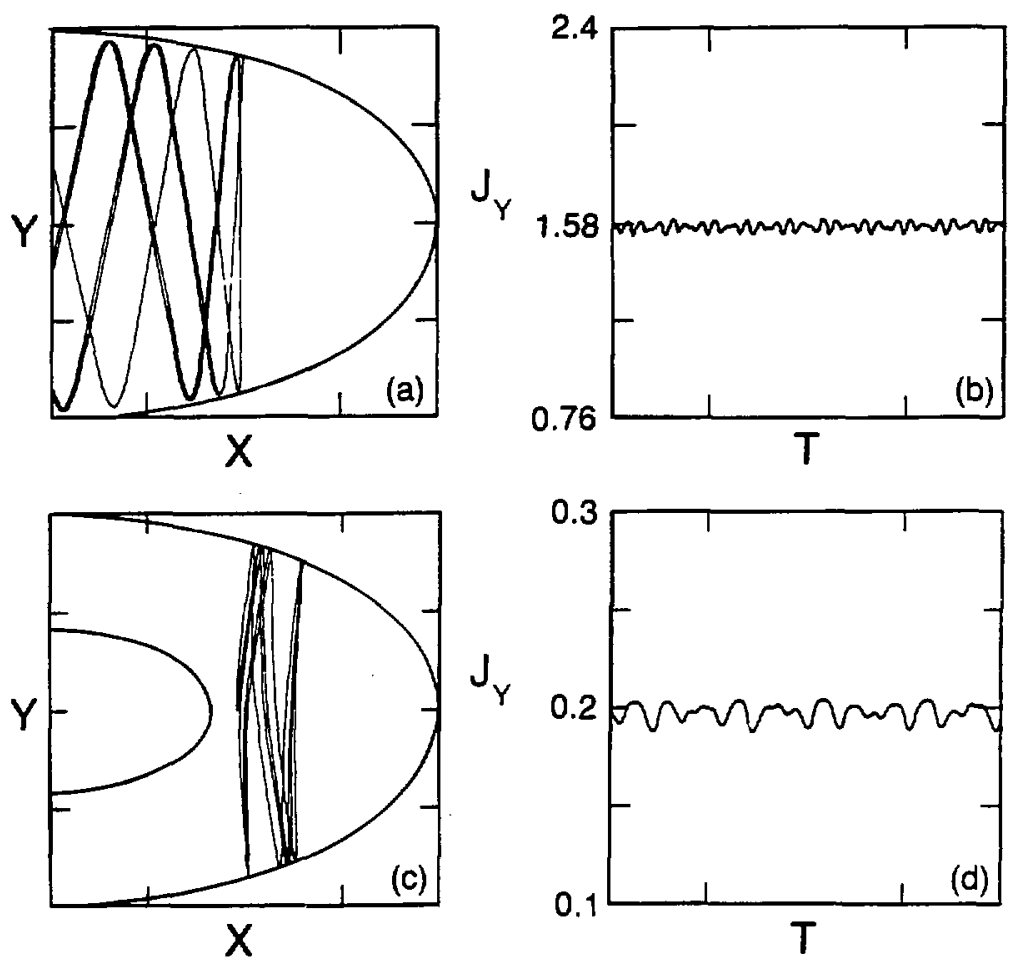

XBL 8310-3331

Figure 3.10 

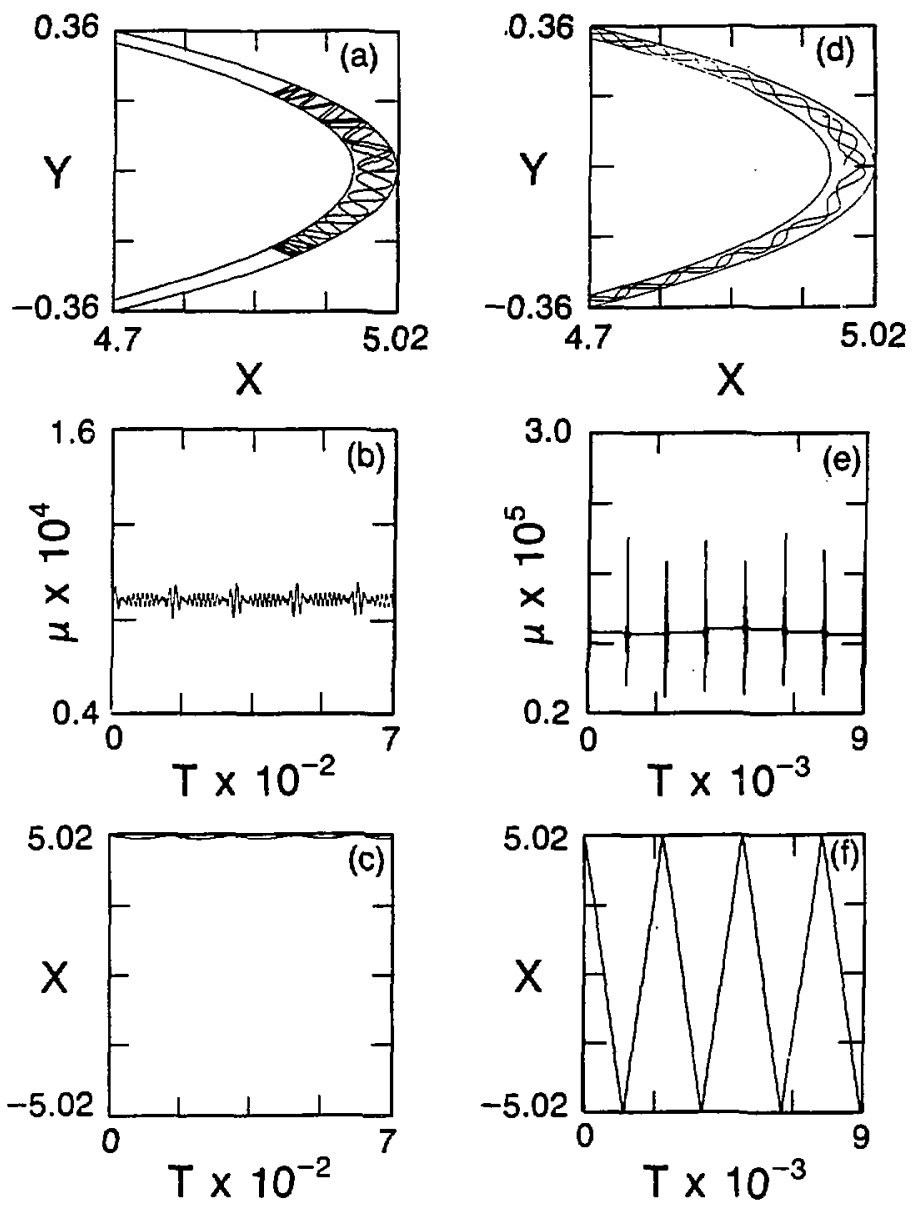

Figure 3.11 XBL 8310-3325 

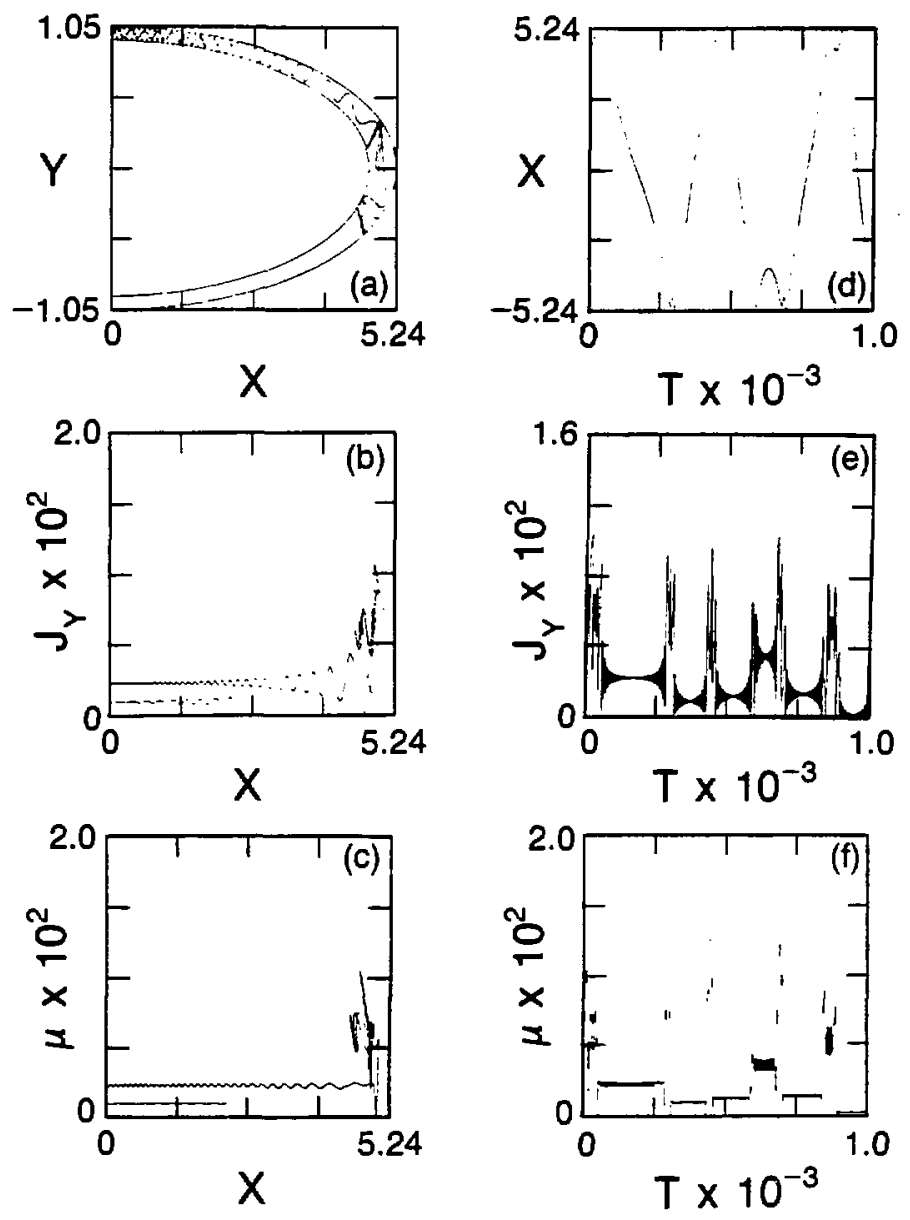

XBL 8310-3335

Figure 3.12 

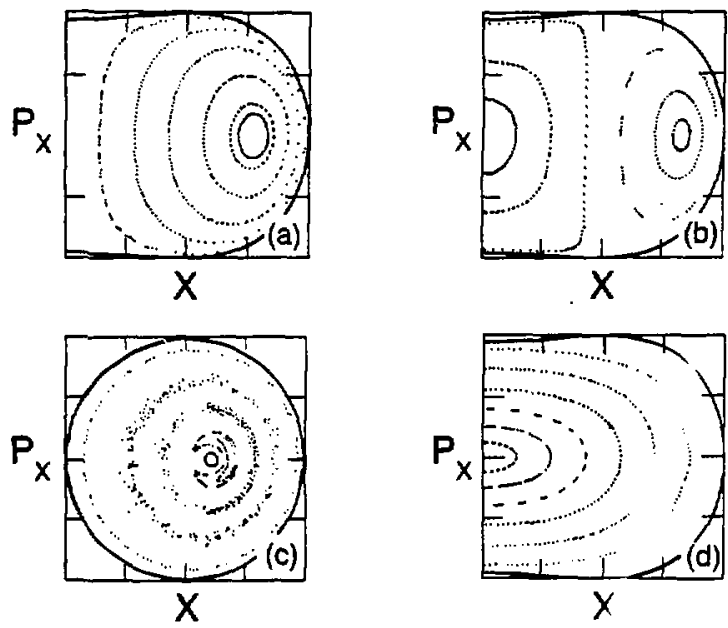

Figure 3.13
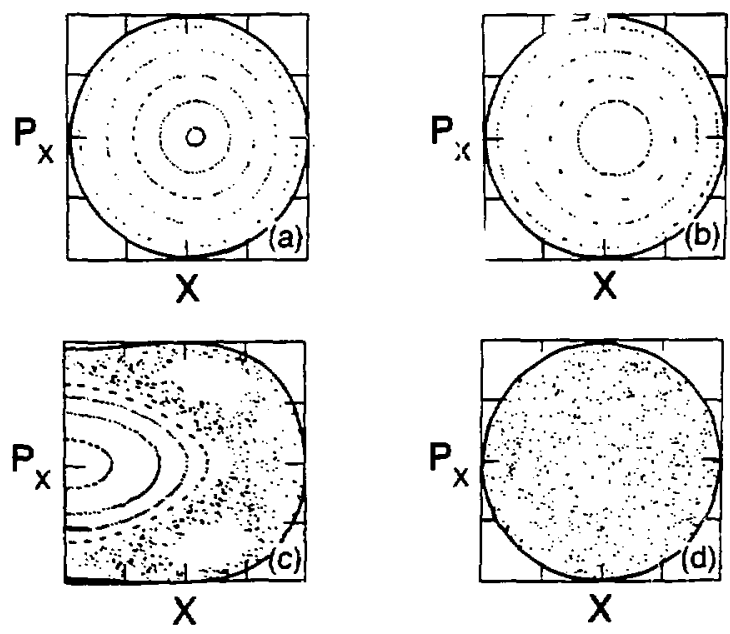

XBL 831033230

Figure 3.14 


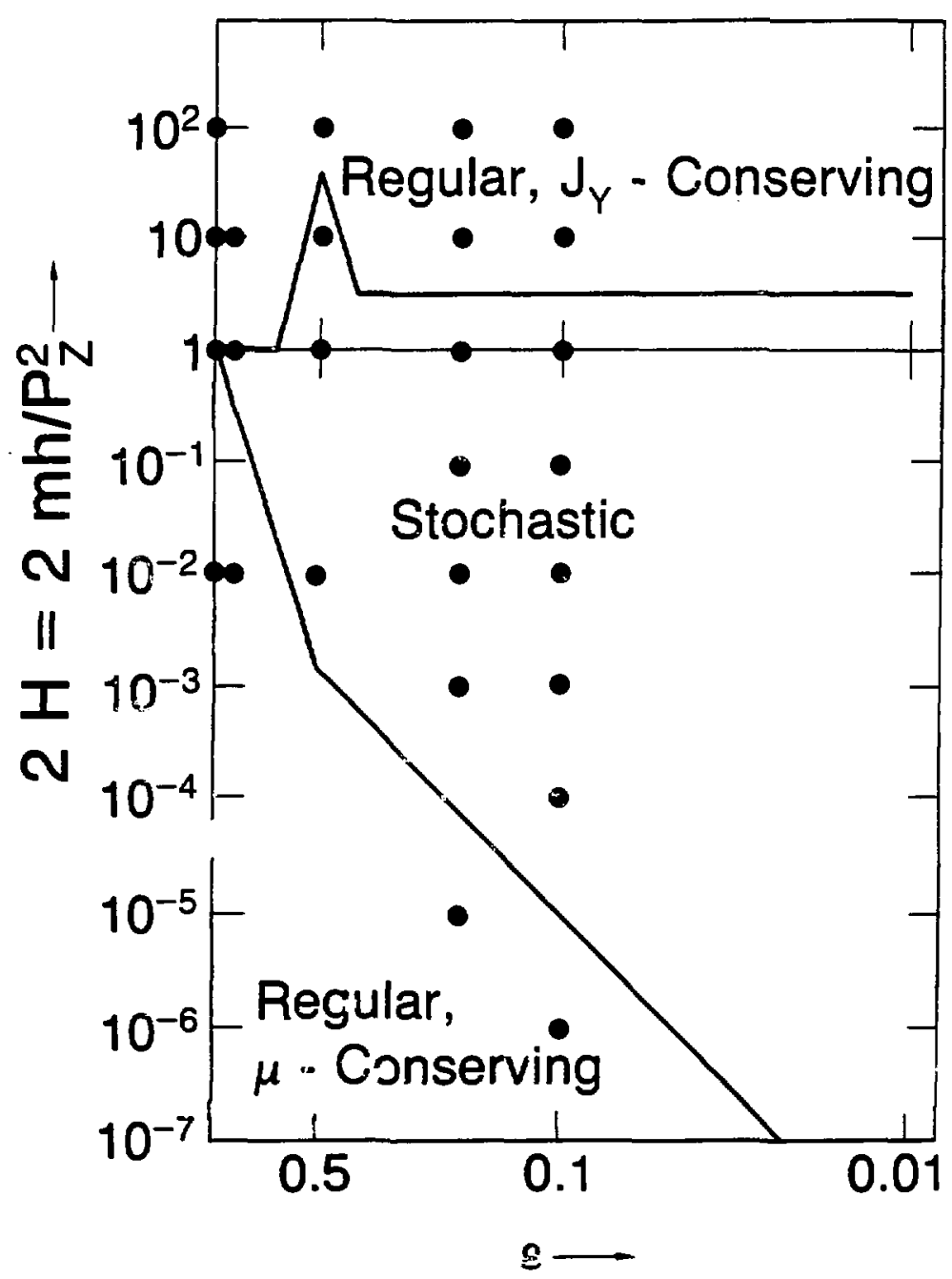

XBL 8310-3326

Figure 3.15 
$\sqrt{2 m h}$

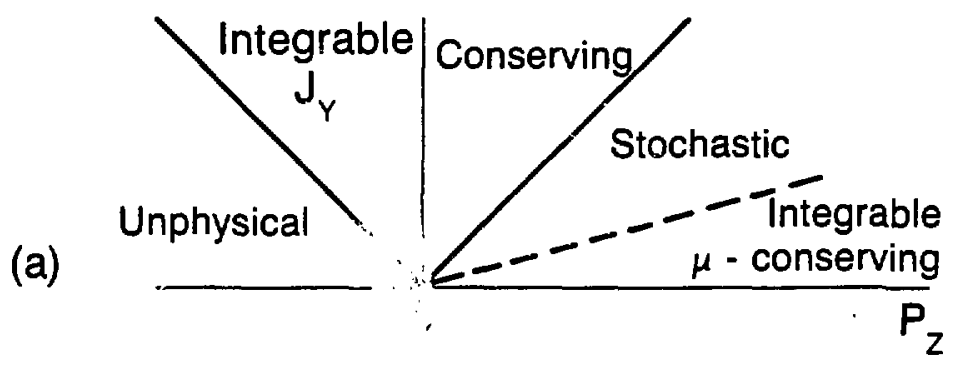

(b)

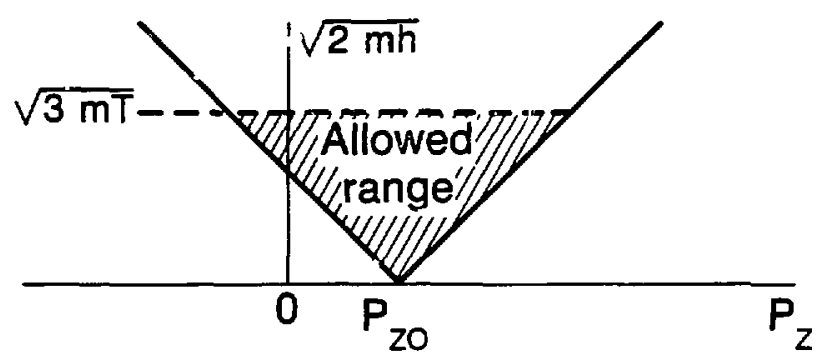

(c)

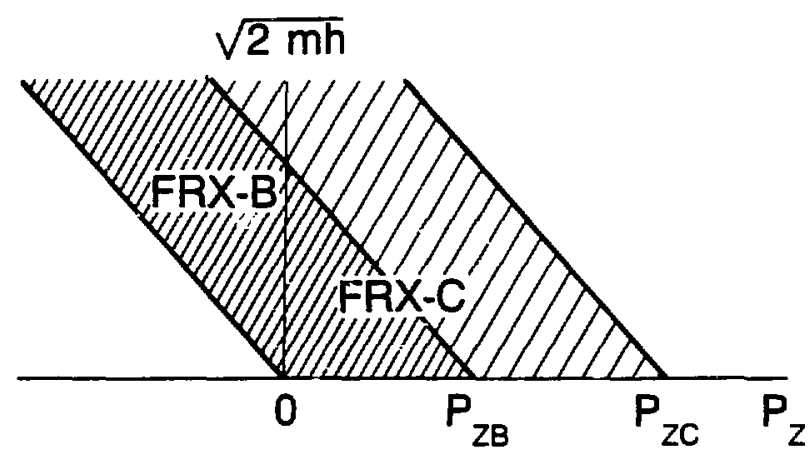

Figure 3.16 


\section{CHAPTER IV \\ THE VLASOY FLUID (VF) MODEL}

\section{A. Introduction}

The VF Model is a low frequency model of a fully ionized plasma in which ions (or the heavier species) are treated as collisionless and electrons (the lighter species) are treated as a massless, pressureless fluid.

It is obvious that the model is most suitable for a system with hot ions and rather cold electrons. In fact many experiments for fusion energy such as FRC are well approximated by such a model. The modei provides a mors realistic description of the gross stability properties of high $\beta$, non-uniform plasma than that of ideal MHD. Inclusion of electron pressure is also possible, although only a limited number of papers $s^{1,2}$ on this subject are available at the moment.

The hybrid model of kinetics and hydromagnetic properties was first suggested by Freidberg in $1972 .^{3}$ He applied the model to a theta pinch with a sharp boundary and found that the growth rate of the $m=2$ mode, where $m$ is the poloidal mode number, can be exponentially smail, but not zero due to the resonant ion orbits, while ideal MHD theory predicts a high growth rate. This may explain why the $m=2$ mode was not seen in the Scyllac experiment.

Several years later, Lewis and Symon $^{4}$ developed a general framework of analyzina the linear stability of an inhomogeneous plasma in 
which there is a collisionless species. They expanded the perturbed quantities in terms of eigenfunctions of the equilibrium Liouville operators. They also introduced a dispersion matrix whose analytic properties determine the nature of the solutions of the initial value problem.

Solving such a dispersion matrix of a VF model, Seyler ${ }^{5}$ examined the stability of a rigidly rotating theta pinch in a one-dimensional model. In particular he compared the growth rates of an asymptotic finite Larmor radius (FLR) thecry and those of an original yF expression without making any approximations. Study of the growth rates as a function of $\mathrm{kr}_{L}$, where $k$ is the axial wave number and $r_{\text {!. }}$ is the gyro radius, shows that the two theories agree very well for $k=0$. However for $k \neq 0$ the destabilizing effect of resonant ions becomes important. Hence, the plot of the growth rates of VF model v.s. $\mathrm{kr}_{L}$ has a long tail beyond the threshold values of $\mathrm{kr}_{L}$ of the FLR theory. This phenomenon is a consequence of the fact that the VF model indicates the same stability condition as that of ideal MHD if the equilibrium ion distribution function is a function of energy only and monotonically decreasing with respect to energy, while FLR theory predicts a more favorable stability criterion than that of ideal MHD.

An extensive study is made of the VF stability in a series of papers. ${ }^{6}$ Also recently, Seyler and Barns ${ }^{7}$ made a small gyro orbit expansion of the VF dispersion functional, taking into account the strong curvature of the magnetic field, and found a stabilizing mechanism to the tilting mode of a FRC. Of course the expansion is not 
valid near the null. They also showed that in the limit of zero gyroradius, their result is in complete agreement with those of Kruskal and Oberman ${ }^{8}$ and Kulsrud ${ }^{9}$.

The dispersion operator $\underset{d}{D}(w)$ appearing in the VF dispersion functional has an interesting feature. ${ }^{4}$ As in Ref. 4 [Lewis and Symon], if one defines the adjoint of the operator $D(\omega)$ in the usual way but treat $\omega$ as if it were real for its conjugation, ${\underset{\sim}{D}}^{+}(\omega)=\left[\underset{\sim}{D}\left(\omega^{\star}\right)\right]^{\dagger}$. (This is the only way to define an adjoint matrix which is an analytic function of $\omega,[D(\omega)]^{+}$is not an analytic function of $\left.\omega.\right)$ Then the matrix elements $\underline{D}_{i j}{ }^{\dagger}(\omega)$ of the adjoint matrix are ${\underset{\sim}{\mathrm{D} j}}^{\dagger}(\omega)=D_{j i}^{D_{j}}(\omega)$. Since the dispersion function of the VF Model has the property that $D_{j i}^{\star}(\omega)=D_{i j}^{D}(\omega)$, we have ${\underset{\sim}{i j}}^{\dagger}(\omega)=D_{i j}(\omega)$ or ${\underset{\sim}{D}}^{\dagger}(\omega)=\underset{\sim}{D}(\omega)$. Because of this Hermitian property of the operator in the sense as described above, the VF disperson functional has a variational nature ${ }^{13}$ of eigenvalue problems. Some of the other interesting properties of the operator are discussed recently by Symon ${ }^{14}$.

In Sec. B, we describe the physical concepts and the approximation made in the model. In Sec. $C$ and $D$ we investigate the equilibrium and the stability properties of the model respectively. Finally, in Sec. $E$, a dispersion matrix is introduced to find the growth rates and the eigenvectors of the VF model. The properties of the matrix are also investigated. As a simple matrix problem, a one by one matrix is considered. In this case, we have to guess an eigenvector, which is a trial function. In the appendix, we mention briefly the basic framework of Symon, Seyler and Lewis ${ }^{4,6}$ as a comparison. 
The VF model and its properties are mostly based on the work of others; the references are noted in the text. My contribution is applying Finn's way of treating stochastic orbits as in Ref. 10 to the VF model, including extension of the method to the treatment of both the regular and the stochastic orbits [part of Sec. D.2] My contribution is, also, in Sec. E.3 which discusses properties of some types of trial functions and the Nyquist diagram approach to select physical growth rates.

\section{B. The Model}

The model applies to a class of fusion experiments that are operated in a regime where ions are collisionless and are well described by the Vlasov equation and yet the collisions among electrons are sufficiently frequent so that the electrons can be described as a fluid on an MHD time scale.

To study plasma stability on the MHD time scale, or longer, and over spatial scales much larger than the Debye length, it is possible to consistently neglect space charge (quasi-neutrality approximation) and the displacement current in Maxwell's equations. The quasineutrality character yields not only the macroscopic ion quantities, but also the electron density as calculated from the appropriate moments of the ion distribution.

Electrons, due to their small mass, are well described by the small and fast gyro-orbits whose guiding centers are tied to the field lines, shorting out any parallel electric fields which tend to arise. 
If we further assume that the electrons are cold and ignore the drag force on electrons due to jons, electrons are governed by the following equation;

$$
\underline{E}+\frac{1}{c} \underline{u}_{e} \times \underline{B}=0 \text {, }
$$

where $\underline{u}_{e}$ is the macroscopic electron velocity. Еq. (4.1) describes the electron motion perpendicular to $\underline{B}$. The motion parallel to $\underline{B}$ is determined from $\underline{B} \cdot \underline{E}=0$ which comes directly from the electron equation of motion.

With these assumptions, after eliminating some unknowns, the basic equations of the $V F$ model are given by

$$
\begin{aligned}
& \underline{\nabla} \times \underline{E}=-\frac{1}{c} \frac{\partial \underline{B}}{\partial t}, \\
& \frac{\partial f}{\partial t}+\underline{v} \cdot \underline{\nabla} f=\cdot \frac{e}{M}\left(\underline{E}+\frac{1}{c} \underline{v} \times \underline{B}\right) \cdot \underline{\nabla}_{\underline{v}}^{f}, \\
& \frac{1}{4 \pi}(\underline{\nabla} \times \underline{B}) \times \underline{B}=e \int\left(\underline{E}+\frac{1}{c} \underline{v} \times \underline{B}\right) f \underline{d v},
\end{aligned}
$$

where $f$ is the ion distribution function and $e$ is the charge of an ion. It is worth noting that $\underline{E} \cdot \underline{B}=0$ is automatically satisfied in Eq. $(4,4)$.

Now, we show that the total energy of the system is conserved by this model. The energy of the system ( $H^{\text {total }}$ ) consists of three 
parts: fieid energy, the kinetic energy of jans and the kinetic energy of electrons.

$$
H^{\text {total }}=\frac{1}{8 \pi} \int \underline{d x}\left(E^{2}+B^{2}\right)+\frac{1}{2} \int \underline{d x d v} M v^{2} f+\frac{1}{2} m \int d \underline{x} n e^{u^{2}}
$$

where $m$ is the electron mass, $n_{e}$ is the number of electrons in unit volume and $e$ is the charge of an ion.

By ignoring the displacement current, the time derivative of - $e$ tốàl energy of the system, in the limit of m going to 0 , reduces to

$$
\frac{d H^{\text {total }}}{d t}=\cdots=\frac{1}{4 \pi} \int \underline{d x} \underline{E} \cdot \underline{J}=0 .
$$

The last equality halds since $\underline{E} \cdot \underline{J}_{e}=0$ which comes from the electron equation of motion described by Eq. (4.1). Hence, the total energy is conserved by the model.

\section{c. Equilibrium}

First we describe the properties of Vlasov Fluid equilibria in general and then find a specific equilibrium of interest. We restrict ourselves to equilibrium ion distribution functions of the form

$$
f_{0}(\underline{x}, \underline{v})=F_{0}(H(\underline{x}, \underline{v}))
$$

where $H=\frac{1}{2} M v^{2}+e \phi_{0}(\underline{x})$ and $\phi_{0}(\underline{x})$ is the equilibrium electrostatic potential. The assumption behind the choice of an equilibrium as a function of energy only is that ion orbits ergodically cover the energy 
surface. If not, each ion orbit separated on the energy surface is assumed to be equally weighted statistically.

For our equilibrium the ion distribution is isotropic, and hence the macroscopic ion velocity is zero; that is, the equilibrium is static with the ions confined electrostaticaliy and all current carried by electrons.

Defining the number density and ion pressure as

$$
n_{0}\left(\Phi_{0}\right)=\int f_{0} d \underline{v},
$$

and $\quad P_{0}\left(\phi_{0}\right)=\int \frac{1}{3} M v^{2} f_{0} d v$,

it follows that

$$
n_{0}=-\frac{1}{e} \frac{d P_{0}}{d \Phi_{0}} .
$$

The force balance equation is obtained from Eq. (4.4)

$$
\begin{aligned}
\frac{I}{4 \pi}\left(\underline{\nabla} \times \underline{B}_{0}\right) \times \underline{B}_{0} & =e_{0} E_{0}, \\
& =e\left(-\frac{1}{e} \frac{d P}{d \phi_{0}}\right)\left(-\Phi_{0}\right), \\
& =\underline{\nabla P}_{0} .
\end{aligned}
$$

Eq. (4.11) indicates that the VF equilibrium is identical to that of ideal MHD for the class of ion equilibrium distributions described 
above. This is because ideal MHD is exact if the pressure is isotropic, as in our equilibrium distribution.

The electrostatic potential can be obtained from the force balance equation. For instance, if we assume that the ion temperature(T) is uniform throughout the plasma, then

$$
e \Phi_{0}(\underline{x})=-T \ln \frac{n_{0}(\underline{x})}{n_{0}(0)} \text {, }
$$

or taking $\Phi_{0}(0)=0$ :

$$
e \phi_{0}(\underline{x})=-T \ln \left(1+\int_{0}^{\underline{x}} \frac{\left(\underline{\nabla} \times \underline{B}_{0}\right) \times \underline{B}_{0}}{4 \pi n_{0}(0) T} \cdot \underline{d l}\right) .
$$

D. Stability

\section{D.1 Gauge Condition and the Variables}

The linearized stability analys is involves the scalar potential $\Phi_{1}$ and the vector potentials $A_{1}$. We are able to eliminate some of the variables by choice of gauge condition and other properties of the VF model.

We define $\underline{\xi}$ such that

$$
\underline{A}_{1}=\underline{\xi} \times \underline{B}_{0}+A_{\|} \hat{b}
$$

where $\underline{\xi}^{-} \underline{B}_{0}=0$ and $\hat{b}=\underline{B}_{0} / \mathrm{P}_{0}$. 
By the definition of Eq. (4.14), the vector $\underline{\xi}$ is the field line displacement.

In most cases a convenient gauge choice is $A_{\|}=0$. This gauge condition cannot be chosen if it is not possible to find a scalar $X$ such that

$$
A_{\|}=\frac{1}{B_{0}} \underline{B}_{0} \cdot \underline{A}_{1}=\frac{1}{B_{0}} \underline{B}_{0} \cdot\left(\underline{A}_{1}^{\prime}-\underline{\nabla} x\right)=0,
$$

or

$$
\underline{B}_{0} \underline{A}_{1}^{\prime}=\underline{B}_{0} \cdot \underline{\nabla X} \text {. }
$$

for any vector $A_{1}^{1}$. The two notable cases in which this gauge is not allowed are (1) when $\underline{B}_{0} \cdot \nabla$ is singular and (2) when there is a periodic constraint on the field line. The former occurs, for example, for the $m=0$ mode in a pure $Z$-pinch, and the latter when $\underline{B}_{0}$ has a closed field line configuration.

The condition $\underline{E}_{1} \cdot \underline{B}_{0}+\underline{E}_{0} \cdot \underline{B}_{1}=0$ can be exprassed as

$$
\underline{B}_{0} \cdot \underline{\nabla}\left[-\Phi_{1}+\frac{1}{c} \underline{u}_{0} \cdot \underline{A}_{1}\right]-\frac{1}{c} u_{0} \cdot \underline{\nabla}\left(\underline{A}_{i} \cdot \underline{B}_{0}\right)+\underline{B}_{0} \cdot \underline{A}_{1}\left[-i_{\omega}-\frac{1}{c} \underline{\nabla} \cdot \omega_{0}\right]=0,
$$

where $\underline{u}_{0}=C \underline{E}_{0} \times \underline{B}_{0} / B_{0}^{2}$. Consequentiy, $\Phi_{2}=\underline{u}_{0} \cdot \underline{A}_{1}$ for the choice of gauge condition $A_{\|}=0$. Here an arbitrary function of $\psi_{0}$, the equilibrium flux, could be added to $\Phi_{1}$ but $\psi_{0}$ doesn't have the dependency of normal mode. Therefore it must be set to zero.

Finally we are left with two variables which are the two components of $\underline{\xi}_{\perp}$. The relationship between $\underline{\varepsilon}_{-1}$ and the viF variables are 


$$
A_{\|}=0, \quad \underline{A}_{\perp}=\underline{\xi} \times \underline{B}_{0} \text { and } \Phi_{1}=\underline{\xi}_{1} \cdot \underline{E}_{0} \text {. }
$$

For a closed magnetic field configuration, the gauge choice $A_{\|}=0$ is not allowed. In this case we choose a gauge by $\Phi_{1}=\xi_{1} \cdot E_{0}$. This gauge is always allowed for a description of unstable modes. Then $A_{\|}=0$ is a restriction of the modes that we are studying. Hence the contribution of $A_{\|}$has to be examined. The condition on $A_{\|}=0 \mathrm{ap}-$ pears exactly the same way as the condition on $\underline{\nabla} \cdot \underline{\xi}=0$. Hence, it is suggestive that for the modes which are well approximated by incompressible displacements then the contribution of $A_{\| l}$ would be small.

In any case, the relationship between $\underline{\xi}_{1}$ and the VF variables shown in Eq. (4.17) will be used throughout the thesis.

\section{D.2 The VF Dispersion Functional}

Now we are ready to express the linearized force balance Eq. (4.4) in terms of $\xi_{1}$ only.

$$
\begin{aligned}
-\underline{F}\left(\underline{\xi}_{1}\right) & +\frac{i \omega e n_{0}}{c} \underline{\xi}_{1} \times \underline{B}_{0} \\
& +i \omega e^{2} \int \underline{d v}\left(\underline{E}_{0}+\frac{1}{c} \underline{v} \times \underline{B}_{0}\right) \frac{d F 0}{d H} \int d t^{\prime} \underline{\xi} \cdot\left(\underline{E}_{0}+\frac{1}{c} \underline{v} \times \underline{B}_{0}\right)=0
\end{aligned}
$$

where $\left.\underline{F}\left(\underline{\xi}_{1}\right)=\frac{1}{4 \pi}\left(\underline{\nabla} \times \underline{B}_{0}\right) \times \underline{B}_{1}+\frac{1}{4 \pi}\left(\underline{\nabla} \times \underline{B}_{1}\right)+\underline{\nabla}_{\underline{E}_{1}} \cdot \underline{\nabla}_{0}\right)$

and $\underline{B}_{1}=\nabla \times\left(\underline{\xi}_{1} \times \underline{B}_{0}\right)$. 
To obtain a dispersion functional we form a dot product of Eq. (4.18) with $\xi^{*}$ and integrate over space, which gives

$$
\begin{aligned}
& 2 \delta H\left(\underline{\xi}_{1}^{*}, \underline{\xi}_{\perp}\right)+\frac{i \omega e}{c} \int d \underline{n}_{0} \underline{\xi}_{\perp}^{*} \cdot \underline{\xi}_{1} \times \underline{B}_{0}+i \omega e^{2} \int d \underline{d v} \underline{\xi}^{*} \cdot\left(\underline{E}_{0}+\frac{1}{C} \times \underline{B}_{0}\right) \frac{d F_{C}}{d H} \\
& x \int d t^{\prime} \underline{\xi}^{*} \cdot\left(\underline{E}_{0}+\frac{1}{c} \underline{\underline{y}} \times \underline{B}_{0}\right)=0 \\
& \text { where } \delta W\left(\underline{\xi}_{\perp}^{ \pm}, \underline{\xi}_{\perp}\right)=-\frac{1}{2} \underline{\xi}_{\perp}^{*} \underline{F}\left(\underline{\xi}_{\perp}\right) \text {. }
\end{aligned}
$$

The quantity $\delta W\left(\underline{\xi}_{1}^{*}, \underline{\xi}_{1}\right)$ is identical to the potential energy variation of ideal MHD for incompressible displacements. It is well known that ideal MHD $6 \mathrm{~W}$ can be written as the sum of two terms:

$$
\delta W\left(\underline{\xi}^{*}, \underline{\xi}\right)=\delta W_{1}\left(\underline{\xi}_{1}^{*}, \underline{\xi}_{1}\right)+\frac{1}{2} \int r_{0}|\underline{\nabla} \cdot \underline{\xi}|^{2} d \underline{x}
$$

where $y$ is the ratio of specific heat capacity at constant pressure to that at constant volume. Notice that $\xi_{\|}$appears only through $|\underline{\nabla} \cdot \underline{\xi}|^{2}$ which is a stabilizing term. Hence $s k$ is minimized by $\underline{\nabla} \cdot \underline{\underline{z}}=0$. However $\underline{\nabla} \cdot \underline{\xi}$ is not a physical quantity but $\xi_{\|}$is. Therefore $\underline{\nabla} \underline{\xi}$ can be made zero as long as one can find a single valued solution for $\xi_{\|}$of $\underline{\nabla} \underline{\xi}=0$ for any given $\underline{\xi}_{L^{-}}$. That is $\xi_{\|}$is given by the solution of $\underline{B}_{0} \cdot \nabla\left(\xi_{\|} / B_{\nu}\right)=-\nabla \cdot \xi_{1}$ which is the form of $\varepsilon q$. (4.15). Hence, if the gauge choice is allowed then the most unstable displacements of ideal MHD are guaranteed to be incompressitle and $\delta W\left(\underline{\xi}^{\star}, \underline{\xi}\right)=\delta W_{1}\left(\underline{\xi}_{1}^{*}, \underline{\xi}_{1}\right)$. Now we express the third term of Eq. (4.21) (we denote this term as $I_{\text {ob }}$ where the subscript "ob" stands for "orbit" so that "I ob stands for orbit integral) in terms of dynamical spectral densities in analogy with 
the approach of Finn 10,11 and Sudan ${ }^{11}$ used in studying the stability of a plasma with an energetic ion beam.

First we introduce $k(z(t))$ as follows where, $z(t) \equiv(\underline{x}(t), \underline{v}(t))$,

$$
e \underline{\underline{E}}_{1} \cdot\left(\underline{E}_{0}+\frac{1}{c} \underline{v} \times \underline{B}_{0}\right) \equiv K(\underline{z}(t)) e^{-i \omega t}
$$

so that $K(z(t))$ is independent of $w$. Then

$$
I_{o b}=i \omega \int \underset{\sim}{d z} \frac{d F_{0}}{d H} e^{2 \gamma t} \int_{-\infty}^{0} d \tau K(\underset{\sim}{z}(t), \tau) e^{-i \omega \tau},
$$

where $\tau=t^{\prime}-t$ and $\left.\mathscr{K}(\underset{\sim}{z}(t), \tau) \equiv K * \underset{\sim}{z}(t)\right) K(\underset{\sim}{z}(t+\tau))$.

Notice that a phase variable of a Hamiltonian system at time $t+\tau$ is determined by the initial phase variable at time $t$ and the time difference $\tau$.

Suppose we have $m$ independent constants of motion, say $I=\left(I_{l}\right.$, $\left.I_{2}, \ldots . I_{m}\right)$, where $m$ is less than or equal to the dimension of the system. It is convenient to transform the 6-dimensional phase variables into $I$ and $I$ where $I$ is the $6-m$ variables; i.e. $d z=J d I d I$ where $J$ is the Jacobian. The transformation from $z(t)$ to $(I, I(t))$ is sim ply a change of variable. It need not be a canonical transformation. $I_{\text {ob }}$ now reads as

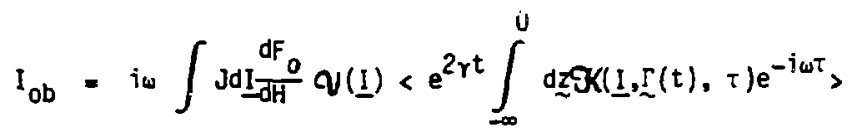


where $Q(\underline{1})$ is the $6-m$ dimensional volume in the phase space accessible to a particle with constants of motion I and the angled bracket $<>$ denotes the average over the $6-m$ dimensional volume, i.e.

$$
\not \bullet=\int \mathrm{d} \Gamma \cdot 1 \int \mathrm{d} \Gamma=\int \mathrm{d} \Gamma \cdot / \mathrm{\sim}(\underline{\mathrm{I}}) .
$$

Since $\underset{\sim}{\sim}$ and $\tau$ are independent variables, we can interchange the order of integration to obtain

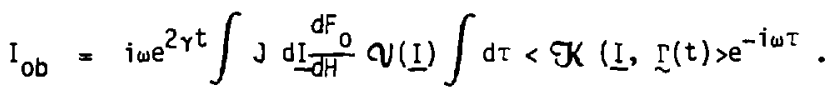

If the Jacobian of the transformation is a function of the variable $I(t)$, then the Jacobian should be kept inside of the bracket. The factor $\mathrm{e}^{2 \mathrm{rt}}$ appears in each term of the dispersion functional and can be cancelled out.

It is important to realize that the quantity $\langle\mathscr{K}(z(t), \tau)\rangle$ is independent $o_{1} i$ whether the motion is ergodic or not as long as the particles are described by a Hamiltonian system. ${ }^{12}$ This is because the volume in the phase space of a Hamiltonian system is invariant by Liouville's Theorem. Since I's are constants of motion, each sub volume in the phase space of given values of I's is invariant, i.e. independent of initial condition. Hence the quantity $\langle\mathcal{T} K(\tau,\langle t), \tau\rangle\rangle$ is independent of $t$.

Because $\langle K\rangle$ is independent of $t$, we can expand it in terms of Fourier integral of $\tau$ :

$$
\left\langle\mathcal{G}(\underset{\sim}{2}(t), \tau\rangle=\frac{1}{2 \pi} \int d B \Phi(B) e^{i B \tau} .\right.
$$


Now the $\tau$-integral in $I_{\text {ob }}$ is carried out easily. Ignoring the initial term of the integral, under the assumption that the imaginary part of $\omega$ is positive, we obtain

$$
\begin{aligned}
I_{o b} & =\omega e^{2 \gamma t} \int d \underline{d B} \frac{d F_{0}}{d H} Q(\underline{I}) \frac{P(B ; I)}{\beta-\omega} \\
& =\omega \int d B \frac{S(\beta)}{\beta-\omega},
\end{aligned}
$$

where $S(B)=e^{2 \gamma t} \int J \underline{I I} \frac{d F_{0}}{d H} Q(\underline{I}) \Phi(s)$

$S(s)$ here is equivalent to the dynamical spectral densities found by Symon, Seyler and Lewis ${ }^{6}$ where they expressed $S(B)$ in terms of eigenvalues and eigenvectors of the unperturbed Liouville operator. As a comparison, their basic framework and the expression of $S(B)$ is briefly discussed in the Appendix.

Finally the VF dispersion functional can be represented in the simple form:

$$
2 \delta \omega+\frac{i \omega e}{C} \int d \underline{n_{0}} \underline{\xi}_{1}^{*} \cdot \underline{\xi}_{1} \times \underline{B}_{0}+\omega \int d \beta \frac{S(\beta)}{\beta-\omega}=0 .
$$

Equation (4.29) has a neat form but it does not show clearly how the kinetic correction appears to $M H O$, since $S(B)$ involves the fluid inertia (the second term of Eq. (4.31)) and the isinetic effects. Because $S(B)$ is the mixture of $M H D$ and the kinetic effects, $S(B)$ may have to be evaluated more accurately than othe:wise necessary to see kinetic corrections to MHD. 
For this reason and to understand the kinetic effects more physically we transform Eq. (4.29) to separate MHO from kinetic. By using the identity

$\left.\underline{E} \cdot e\left(\underline{E}_{0}+\frac{1}{c} \underline{v} \times \underline{B}_{0}\right)=\underline{E} \cdot \underline{F}=M \underline{\underline{E}} \cdot \frac{d \underline{v}}{d t}=M \frac{d(\underline{\xi} \cdot \underline{v})}{d t}-\underline{v} \cdot \frac{d E}{d t}\right)$,

the orbit term, the third term of Eq. $(4.21)$, can be transformed as follows;

$$
\begin{aligned}
& I_{o b} \equiv i \omega \int d z \underline{\xi}^{\star} \cdot \underline{F} F_{0}^{\prime}(H) \int d t^{\prime} \underline{\underline{E}} \cdot \underline{F} \\
& =i \omega \int d z \underline{\xi}^{\star} e\left(\underline{E}_{0}+\frac{1}{c} \underline{v} \times \underline{B}_{0}\right) F_{0}^{\prime}(H)\left[\underline{\xi} \cdot \underline{v}-\int d t^{\prime}(\underline{v v}: \underline{\nabla \xi}-i \omega \underline{v} \cdot \underline{\xi})\right]
\end{aligned}
$$

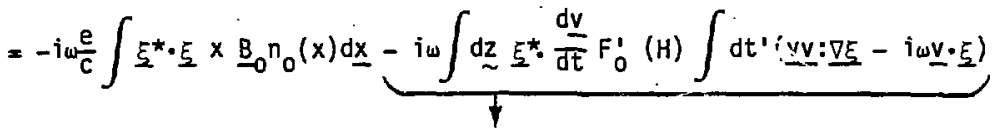

$$
\begin{aligned}
& \underbrace{\left.-i \omega \int d z F_{0}^{\prime}(H) \frac{d}{d t}\left(\xi^{*} \cdot \underline{v}\right)-\underline{v} \cdot \frac{d \xi^{*}}{d t}\right) \int d t^{\prime}(\underline{v v}: \underline{\nabla \xi}-\omega \underline{w} \cdot \underline{\xi})} \\
& \underbrace{-i \omega \int d z} F_{0}^{\prime} \frac{d}{d t}\left(\xi^{*} \cdot \underline{v}\right) \int d t^{\prime}(\underline{v v}: \underline{\nabla}-i \omega \underline{v}-\underline{\xi}) \\
& +i \omega \int d z F_{0}^{\prime}(H)(\underline{v}: \underline{\nabla} \underline{\underline{z}}-i \omega \underline{v}-\underline{\xi})^{*} \int d t^{\prime}(\underline{v v}: \underline{\nabla \xi}-i \omega v-\underline{\xi}) \\
& -i \omega \int d z F_{0}^{\prime} \frac{d}{d t} \underline{\xi}^{*} \cdot \underline{v} \int d t^{\prime}(\underline{v v}: \underline{\nabla} \xi-i \omega \underline{\underline{v}}-\underline{\xi}) \\
& \text { (1) }
\end{aligned}
$$

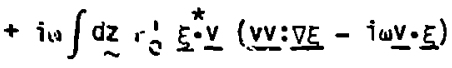

$$
\begin{aligned}
& \text { (2) (3) }
\end{aligned}
$$


The term (1) transforms further as $-i \omega \int d H d P F_{0}^{\prime} \int d \underline{\partial} \frac{d}{d t}\left[\int d t^{\prime} \underline{\xi}^{\star} \cdot \underline{v}(\underline{v v}: \underline{\nabla \xi}-i \omega \underline{v} \cdot \underline{\xi})\right]$. The integration. limits of $\underset{\sim}{\Gamma}$ are only functions of $H$ and $P_{z}$. Therefore $\int d \underset{\sim}{\Gamma}$ can be moved to the right of $\frac{d}{d t}$. Also since $\Gamma_{\sim}^{\prime} s$ are independent to $t^{\prime}$, the $\Sigma$ integral can be moved to the right side of $\int d t$ ' yielding the term (1) as

$$
-i \omega \int d H d P_{z} F_{0}^{\prime} \frac{d}{d t} \int d t^{\prime} \int d \Gamma \underline{\xi}^{*} \underline{v}(\underline{v v}: \underline{\nabla \xi}-i \omega \underline{\underline{\xi}} \underline{\underline{\xi}})
$$

for particles governed by Hamiltonian dynamics. The term 1 after the integral is only a function of the time difference of the two time correction. Therefore the term (1) becomes

$$
-i \omega \int d H d P_{2} F_{0}^{\prime} \frac{d}{d t} \int_{-\infty}^{0} d \tau \mathcal{K}\left(H, F_{z}, \tau\right),
$$

which is clearly a constant, independent of $T$, after the $\tau$-integral and thus vanishes upon the derivative with respect to $t$. The term(2) also vanishes because of the odd parity in velocity. The third terin becomes the inertia term;

$$
\begin{aligned}
-i \omega \int \underset{\sim}{d z F} \dot{\xi}_{0}^{*} \cdot \underline{v}(-i \omega \underline{v} \cdot \underline{\xi}) & =-\frac{\omega^{2}}{T} \int d z F_{0}|\underline{v} \underline{\underline{\xi}}|^{2} \\
& =-\omega^{2} \int|\xi|^{2} n_{0}(\underline{x}) d \underline{x} .
\end{aligned}
$$


Collecting ail the terms together, we obtain

$$
\begin{aligned}
& I_{o b}=-i \omega \frac{e}{c} \int \xi^{*} \xi \times \underline{B}_{0} n_{0} d x-\omega^{2} \int|\underline{\xi}|^{2} n_{0}(\underline{x}) d \underline{x}
\end{aligned}
$$

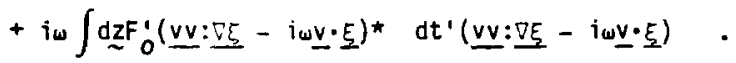

The new form of the dispersion functional is then

$$
2 \delta W-\omega^{2} \int|\xi|^{2} n_{0}(x) d x+i \omega \int d z F_{0}^{\prime}(\underline{v v}: \underline{\nabla \xi}-i \omega \underline{v} \cdot \underline{\xi})^{\star} \int d t^{\prime}(\underline{v v}: \underline{\nabla \xi}-i \omega \underline{v} \cdot \underline{\xi})=0
$$

or

$$
25 w-\omega^{2} \int|\xi|^{2} n_{0}(x) d x
$$

$$
+\omega\left[\int d B \frac{S_{1}}{B-\omega}+\omega\left[\int d B \frac{S_{2}}{\beta-\omega}+\left(\int d B \frac{S_{2}}{s-\omega}\right)^{\star}\right]+\omega^{2} \int d B \frac{S_{3}}{e-\omega}\right]=0
$$

where $S_{1}$ and $S_{3}$ are the two time correlation of $\underline{v v}: \underline{\nabla} \underline{G}$ and $\underline{v} \cdot \underline{\xi}$ respectively, and $S_{2}$ is the cross correlation of $(\underline{v v:} \underline{\nabla \xi})^{*}$ and $\underline{v} \cdot \underline{\xi}$.

\subsection{Stadility Theorems}

Even though the detajled expression of the dynamical spectral density $S(B)$ is different, the dispersion function is exactiy the same as that of Seyler and Lew: $s^{6}$. Therefore, the sufficient and necessary conditions for stability can be investigated in the same way as they did.

First we set the real and the imaginary part of Eq. (4.29) to zero respectively to obtain 


$$
\begin{gathered}
91 \\
2 \delta W-\left(\sigma^{2}+\gamma^{2}\right) \int d \beta \frac{S(\beta)}{(\beta-\sigma)^{2}+\gamma^{2}}=0 \\
i \frac{e}{c} \int d \underline{x} n_{0} \xi^{*} \cdot \underline{\xi} \times \underline{B}_{a}+\int d \beta \frac{\beta(\beta(\beta)}{(\beta-\sigma)^{2}+\gamma^{2}}=0,
\end{gathered}
$$

where $\sigma$ is the real part and $Y$ is the imaginary part of $\omega$. We have used the fact that ow is real and the integral term $\int \frac{d x_{F}^{*}}{F_{5}} \underset{\xi_{0}}{ }{ }_{B}$ is purely imaginary.

Sufficient Condition for Stability: From Eq. (4.32a), it is apparent that there is no solution for $\mathrm{Y}$ if $\delta \mathrm{W}>0$. Recalling that the expression (4.32) is valid only for $Y>0$, we conclude that $\delta W>0$ implies $r<0$.

Necessary Condition for Stability: First we study the behavior of Eq. (4.32) in the limit $\gamma \rightarrow 0$. Using an identity

$$
\lim _{\gamma \rightarrow 0} \frac{1}{\pi} \frac{\gamma}{c^{2}+y^{2}}=\delta(c),
$$

for any real $c$, we obtain an expression from Eq. (4.32a) by taking the limit of $\gamma \rightarrow 0$,

$$
2 \delta W-\frac{\pi\left(\sigma^{2}+\gamma^{2}\right)}{\gamma} S(\sigma)=0 ; \text { as } \gamma \rightarrow 0
$$

Here, we assume that $S(\sigma)$ does not vanish, which in general is a weak assumption. With this assumption Eq. (4.33) requires that $\lim _{\gamma \rightarrow 0} \frac{\sigma^{2}}{\gamma}$ should be finite since $\delta W$ is finite. Therefore $\sigma$ should appraach zero 
as $y$ does. This means that the marginal stability corresponds to $\omega=0$. Furthermore, it also implies that $Y=0$ only at $\omega=0$. Hence, it is only necessary to consider the sign of $\delta \mathrm{W}$ near $\omega=0$ in order to establish a necessary condition on the sign of sW which will also be valid arbitrarily far from the marginal point. Therefore again from the near marginal expression Eq. (4.33), it is apparent that if there is a solution $r<0$ then $\delta W>0$.

\section{E. Calculation of Growth Rates}

So far we have discussed the stability only qualitatively. Our next task is to find the growth rates of the unstable modes, if any. From Eqs. (4.32a and b) there seems to be an w that satisfies those equations for almost any $\xi$ since there are two unknowns a and $\gamma$ $\left(\omega=\sigma+i_{y}\right)$ and there are two equations $(4.32 a)$ and $(4.32 b)$. What do $w$ and $\xi$ mean in this case? We will answer tho question in the following sections.

\section{E.1 The Dispersion Matrix}

Let us express the linearized force equation in terms of a dispersion operator $0(\omega)$, as

$$
\underset{\sim}{D(w)} \cdot \underline{\xi}=0
$$

Then the VF dispersion functional of Eq. (4.29) has a form of

$$
\left(\underline{E}^{\star}, \underline{\sim}(\omega) \underline{\xi}\right)=0
$$


93

where the bracket is a short notation of the integration over various variables as shown in Eq. (4.29). Treating $\underline{\xi}^{*}$ and $\underline{\xi}$ as independent variables, we recover Eq. (4.34) as a stationary condition of the dispersian functional with respect to the variation of $\underline{\xi}^{\star}$. The stationard condition with respect to the variation of $\underline{\xi}^{\star}$ requires that

$$
\left(\delta \underline{\xi}^{*}, \underline{D \xi}\right)=0 \text { for any } \delta \underline{\xi}^{*} \text {, }
$$

which is possible only when $\underline{D}(\omega) \cdot \underline{\xi}=0$.

With the above basic knowledge, let us expand $\underline{\xi}$ in terms of a complete but not necessarily orthogonal set of basis functions $\eta$ :

$$
\begin{aligned}
& \underline{\xi}=\sum_{\underline{l}} a^{a} \underline{\ell}^{n} \underline{l} \text {, } \\
& \text { Likewise } \underline{\xi}^{*}=\sum_{\underline{\ell}^{\prime}} a_{\ell^{\prime}}^{*} \eta_{\ell^{\prime}}^{*} \text {, then }
\end{aligned}
$$

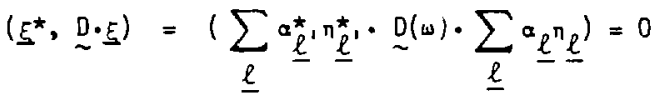

and

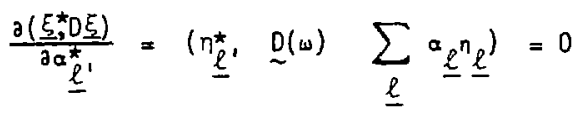

If we demand that Eq. (4.38) is satisfied for any bast is function of $\eta_{\ell}$ then we recover the force $E q$. (4.34), since the $n_{\ell}$ 's form a complete set.

More explicitly Eq. (4.38) is

$$
\left(n_{\ell^{\prime}}^{*} \stackrel{D n_{1}}{\sim}\right) a_{1}+\left(n_{\ell^{\prime}}^{*} \stackrel{D}{\sim} n_{2}\right) a_{2}+\ldots=0
$$


where $\ell^{\prime}=1,2,3, \ldots$, where the vectors $\underline{\ell}$ and $\underline{\ell}^{\prime}$ are represented by an indecies $\underline{\ell}$ and $\underline{\ell}^{\prime}$ for simplicity, which corresponds to a case where the trial function is represented by a one component vector. Eq. (4.38) or (4.38) is simply a set of homogeneous equations. The problem is then reduced to an eigenvalue problem of a matrix whose element is

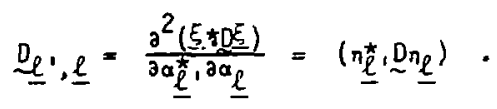

Notice that each element of the VF dispersion matrix requires the detailed information of the equilibrium ion orbits.

In an approximate treatment, or a numerical calculation, completeness means that there must be enough basis functions so that the true eigenfunctions can be adequately represented by a linear combination of basis functions chosen. By solving the dispersion matrix, we obtain the dispersion relation of the system with the errors which arise due to the number of basis functions.

\section{E.2 The Properties of VF Dispersion Operator}

Before one undertakes a complicated computation, it is extremely useful to know the properties of the matrix. First we define an adjoint operator

$$
\underline{D}^{+}(\omega)=\left[D\left(\omega^{\star}\right)\right]^{\dagger} .
$$

That is, the complex conjugation of $D(\omega)$ is obtained, treating $\omega$ as if it were real. By this definition $D(\omega)$ is analytically continuous on 
the rea] axis. [See Ref. 4.] From Eq. (4.29) and the definition of $S(B)$ in Eq. (4.28), and Eq. (4.41), it is clear that the VF dispersion matrix functions satisfy $\underline{Q}^{+}(\omega)=\underline{Q}(\omega)$. Therefore the VF dispersion operator is Hermitian with the definition of Hermitian matrix function as $\underline{D}^{+}(\omega)=\underline{Q}(\omega)$. The normal mode frequencies $\omega$ are the roots of the dispersion relation

$$
\operatorname{det} \underline{Q}(\omega)=0 \text {. }
$$

From the Hermitian property of $D(w)$ and the definition (4.40), the component of the matrix

$$
\underline{\sim}_{i j}(\omega)=\underline{\sim}_{i j}^{\dagger}(\omega)=\underline{\sim}_{j i}^{*}(\omega)=\left[D_{j i}\left(\omega^{*}\right)\right]^{\dagger} .
$$

Therefore, interchanging the rows and columns and taking the complex conjugate, we obtain

$$
\operatorname{det} \underset{\sim}{Q}\left(\omega^{\star}\right)=0 \text {. }
$$

This shows that $\omega^{\star}$ is also a root of the dispersion relation if $\omega$ is. Suppose the system has translational symmetry in $z$. Then the dispersion matrix are diagonalized with respect to $k$ where

$$
\xi-\sum_{t,-} e^{ \pm i k z-i \omega t}
$$

Thus the dispersion matrix is decomposed into two parts: one with eigenvectors with $+k$ and the other with $-k$. 
The relation of the normal mode eigenfrequencies between the two decomposed dispersion matrices is obtained by following our definition of the conjugate of a function:

$$
\xi(-\omega,-k)=\xi^{\star}(\omega, k),
$$

The adjoint equation of $\underline{Q}(\omega, k) \cdot \underline{\xi}(\omega, k)=0$ is $[\underline{Q}(\omega, k)]^{\dagger} \cdot \underline{\xi}(\omega, k)=0$. By the Hermitian property of $D$ and the relation (4.46),

$$
\underline{Q}\left(\omega^{\star}, k\right) \cdot \underline{\xi}(-\omega,-k)=0 \text {, or } \underline{Q}\left(-\omega^{*}, k\right) \cdot \underline{\xi}(\omega,-k)=0 .(4.47)
$$

This shows that if $\omega=0 \pm$ iy are the eigenfreqencies of the eigenvector with $k$, then $\omega=-\sigma \pm$ is are the eigenfrequencies of the eigenvector with $-k$ or vice versa. This is inysically understandable since the stability does not depend on the direction of the propagation of the perturbation; i.e., only the real frequency has the opposite sign. Therefore, if the system has ignorable coordinates, we only have to evaluate using eigenvectors with $+k$. The eigenfrequencies of $-k$ can be deduced by analogy. Also, a straightforward calculation shows a nice symmetric property:

$$
S^{*}(\beta, k)=-S(-\beta,-k)
$$

and

$$
D^{*}(w, k)=\underline{Q}(-w,-k) \text {. }
$$

The symmetric property can be extended trivially for a system with many ignorable coordinates. Furthermore the property that not only $\omega^{\star}$ 
but also $-\omega^{\star}$ is a solution of the dispersion relation if $\omega$ is a solution, is true in general regardless of whether the system has ignorable coordinates or not.

Since a physical system is described by real functions of $\xi$, consider $\underline{\xi}$ whose spatial dependent part of $\underline{\xi}$ is real, more precisely $\underline{\xi}(\underline{x}, \omega)=\underline{\xi}(\underline{x}) e^{-i \omega t}$ where $\underline{\xi}(\underline{x})$ is real. Then from the dispersion functional relation $[Q(\omega)]^{\dagger} \cdot \xi^{\star}=0$. Following our defitition of complex conjugate, namely treating $w$ as if it were real in the process, the last equality leads to $\underline{\underline{Q}}\left(-\omega^{\star}\right) \cdot \underline{\xi}(\underline{x}, \omega)=0$ since $\xi^{\star}(\underline{x}, w)=\underline{\xi}(\underline{x},-w)$ and $[Q(w)]^{\dagger}=Q\left(w^{\star}\right)$. Therefore $-w^{*}$ is also the solution of the dipersion relation (4.42) if $\omega$ is so. Combining with the previous results we conclude that the eigenfrequency $w$ of the dispersion relation (4.42) appear in the following forms:

$$
\omega= \pm i_{\gamma}, \omega= \pm_{\sigma} \text { or } \omega= \pm_{\sigma} \pm i_{\gamma},
$$

where $\sigma$ and $\gamma$ are real representing the real frequency and the growth rates (or the damping rates) of the eigenvectors. Remember that the damping mod; are not physical since the relation (4.42) is obtained under the assumption that the mode is growing.

\section{E.3 Trial Function Approach}

Evaluating exact growth rates from a VF dispersion matrix requires in general, encrmous numerical wor ept in a very special or trivial case. This is because each element of the matrix needs the detailed information of the orbits. Hence it is suggestive to make the matrix as small as possible as a start. 
Especially if we are interested in finding some kinetic corrections to ideal MHD for some particular modes, it is reasonable to use the MHD eigenvectors as trial functions and find the expectation values of the growth rates of the VF dispersion functional. This corresponds to a one by one matrix. The trial function approach of a set of ideal MHD eigenvectors will predict the correct transition near $\omega=0$ since the two models have the same stability criterion.

The justification of a trial function approach is based on the fact that the errors of the expectation values of $\&$ to the actual eigenvalue $\omega$ of the VF model are less sensitive than the errors in trial functions to $V F$ eigenvectors. ${ }^{12}$ It is in general not at all clear whether the above idea is applicable if the operator is not Hermitian. Fortunately our dispersion operator is Hermition in the sense of Lewis and Symon and has a variational nature as fiscussed in the previous section. However, our problem is in fact that we do not know what the zeroth order eigenvectors are until we solve an exacs matrix problem.

In short, for a chosen trial function whose value of $s \mathrm{~W}$ is negative there always exists a solution, with growth rates, corresponding to the function from Eqs. (4.32a) and (4.32b). If the function is close to the true eigenvectors of the VF model the growth rates are reliable and show the ortit effect correctly. If the trial function is way off the true modes, then the growth rates found by the procedure are not physical. Hence for a trial function approach, it is crucial to find a good trial function on physical grounds.

Since the structure of the modes depend on the detailed configuration of the system: we will simply consider two general categories of 
trial functions; standing wave displacements and the travelling wave displacements.

(1) Travelling Wave Trial Functions: $\xi=\xi(x) e^{-i \omega t}$ where $\xi(x)$ is complex. This set of trial functions describes perturbations propagating in some spatial directions. For example, if $\underline{\xi}=\xi(x, y) e^{i k z-i \omega t}$, then the displacement has nodes in $x$ and $y$ but propagates in $z$ (travelling wave). This choice is most appropriate when there is a spatial symmetry. For a travelling wave trial funcrion, we have to solve Eqs. (4.32a) and (4.32b) simultaneously for $\sigma$ and $Y$ since $S(B)$ is no longer symmetric in 8 .

In order to understand the properties of the dispersion functional, we drat Nyquist diagrams. First define a complex function $G(\omega)$ as

$$
\operatorname{Rea}[G(\omega)]=\left(\sigma^{2}+\gamma^{2}\right) \int d \beta \frac{[-S(\beta)]}{(\beta-\sigma)^{2}+\gamma^{2}},
$$

and

$$
\text { Ima }(\omega)]=\int d \beta \frac{\beta S(\beta)}{(\beta-\sigma)^{2}+\gamma^{2}}
$$

so that Eq. (4.32) becomes

$$
\begin{aligned}
& 2 \delta \omega+\operatorname{Real}[G(\omega)]=0, \\
& I_{c}+\operatorname{Imag}[G(\omega)]-0,
\end{aligned}
$$

where $I_{c}=i \frac{e}{c} \int d \underline{x}_{0} \underline{\xi}^{\hbar} \underline{\xi} \times \underline{B}_{0}$ which is real.

For a given $\gamma$, we plot $\operatorname{Imag}[G(\alpha)]$ on the vertical axis and Real $[G(\omega)]$ on the horizontal axis by changing a from $-\infty$ to $+\infty$. Notice 
that $r$ appears in Eq. (4.32) only as $r^{2}$. Of course negative values of $\gamma$ are meaningless since the Eq. (4.32) holds only for positive $r$. For example, suppose that che spectral density $S(B)$ is obtained as in Fig. 4.1(a). From the spectral density, the Nyquist diagrams of three different $r^{2}$ values are drawn in Fig. 4.1(b) where the dotted lines are for the positive $\sigma$ values and the solid lines are for negative $\sigma$ values.

As $\sigma \rightarrow \pm \infty, \operatorname{Imag}[G(\omega)] \rightarrow 0$ and Real $[G(\omega)]$ approaches to a fixed value $-\int \mathrm{OB} S(\beta)$ for any $\gamma^{2}$. Therefore all the Nyquist diagrams merge to a point as $a \rightarrow \pm \infty$. The diagram becomes large as $r^{2}$ decreases.

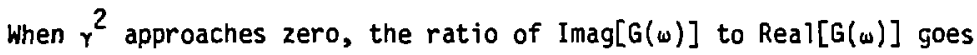
to either $+\infty$ or $-\infty$ depending on the sign of $\sigma$ as $\sigma$ approaches zero. As $\sigma \rightarrow \pm_{\infty}$, the ratio becomes zero. Therefore, the Nyquist diagram of $\gamma^{2} \rightarrow 0$ starts from somewhere on the imaginary axis (the value on the imaginary axis depends on the structure of $S(\beta))$ and follows minus imaginary axis as a increases from zero. The diagram encircles the fourth quadrant of $G(\omega)$ and approaches to the fixed point $\left(0,-\int d_{B} S(B)\right)$ following the real axis from infinity. Similarly the curve of $\sigma<0$ encircles the first quaridrant of $G(w)$ and makes a closed contour with that of $a>0$. This diagram is sketched in Fig. 4.1(b). If wi consicer Nyquist diagrams of all different values of $\gamma^{2}$, it will cover the whole right half plane of $G(w)$. The solution of $E q .(4.32)$ is the value of $\sigma$ and $Y$ at the point of $\left(-2 \delta W,-I_{c}\right)$ on $G(\omega)$ plane. Therefore, if there is a $\underline{\xi}$ which makes $\delta W<0$, then a solution for $\gamma^{2}$ exists in general which shows the instability. This is consistent with the stability theorem disclissed in Sec. D.3. 
(2) Standing Wave Trial Functions: $\xi=\xi(x) e^{-i \omega t}$ where $\xi(x)$ is real. This set of trial functions describes displacements whose fluid element oscillates with fixed nodes.

For a standing wave trial function, the first term of Eq. (4.32b) vanishes and so we deduce $\sigma=0$ from the fact that $S(B)<0$ (not zero) and symmetric in $\beta$. The growth rates are then simply the solution of the following equatio:

$$
2 \delta W-\gamma^{2} \int d \beta \frac{S(\beta)}{\beta^{2}+\gamma^{2}}=0 .
$$

Notice that the second term of $\mathrm{Eq} .(4.51)$ is monotonically increasing with respect to $\gamma^{2}$ from 0 to $-\int d B S(\beta)$. Therefore if $\int \mathrm{dBS}(B)<\delta W<0$, then there is always an unstable mode and only one. Otherwise the dispiacement is not unstable.

The Nyquist diagram in this case is symmetric with respect to the real axis since the spectrum $S(B)$ is symmetric in $\beta$. So the domain of $G(\omega)$ is $\left[0,-\int d_{B} S(\beta)\right]$ on the real axis. This is of measure zero compared to that of a travelling wave trial function case.

Since the domain of $G(\omega)$ for a standing wave trial function does not cover the whole positive real axis, the existence of a standing wave trial function which makes oW negative does not imply a solution for $\gamma^{2}$ of the VF dispersion functional. In other words a standing wave displacement which is unstable with respect to ideal MHD can be stable in VF picture. Nevertheless, this is not contradictory to the stability theorem of Sec. D3, since the system is still unstable, 
which can be easily seen by simply using a travelling wave form of the same displacement. The behavior of a standing wave displacement is rather singular in the VF picture. 


\section{APPENDIX A}

The same VF model has been studied by Symon, Seyler and Lewis 4,6 by expanding the perturbed distribution function $f_{1}$ in terms of eigenvalues $\left(i \mu_{r}\right)$ and eigenvectors $\left(w_{r}\right)$ of the equilibrium Liouville's operator $\left(\mathscr{S}_{0}\right)$. We will illustrate the major scheme of their work just for a comparison with our work.

Since $\mathscr{H}_{0}$ is an anti-Hermitian operator the eigenvalues of the operator are purely imaginary. We write the eigenvalues as i $u_{r}$ so that $\mu_{r}$ is real. Then

$$
\mathscr{P}_{0} W_{r}=i_{\mu_{r}} W_{r} \text {. }
$$

The index $r$ stands for a list of indices needed to specify a unique eigenfunction. In terms of the eigenvectors $\left(W_{r}\right)$ of the unperturbed Liouville's operator $\left(\mathscr{G}_{0}\right)$, one can write

$$
f_{1}(q, g, t)=\sum_{r} \gamma_{r}(t) W_{r}(g, g),
$$

where $\underline{p}, \underline{a}$ are phase space variables and $\gamma_{r}(t)$ is the coefficient of each component and real. One can also make Laplace transformations to all functions of time as follows:

$$
\hat{E}(\omega)=\int_{0}^{\infty} \underline{\xi}(t) e^{i \omega t} d t .
$$


The basic idea of using the expression of (A3) is essentially the same as the normal mode representation used in the main text where $\underline{\xi}=\underline{\xi}(\underline{x}) e^{-i \omega t}$. The only difference of these two notations is the factor $e^{2 \gamma t}$. The factor appears in every term in the dispersion functional derived in the text and thus cancels out at the end, while the factor never appears if one uses the expression of (A3).

$$
(\hat{\xi}, D, \underline{\xi})=-2 \delta W-i \omega \frac{e}{c} \int d \underline{x} n_{0} \underline{\underline{\xi}} \underline{\hat{\xi}} \times B_{0}-\omega \int d B \frac{S(\beta)}{\beta-\omega},
$$

where

$$
S(\beta)=e^{2} \sum_{r} \delta\left(\mu_{r}-\mu\right) \frac{d F_{0}(H)}{d H}\left|\int d \underline{d} \underline{P} \underline{\hat{\xi}^{\star}} \cdot\left(\underline{E}_{0}+\frac{1}{c} \underline{\underline{N}} \times \underline{B}_{0}\right) W_{r}\right|^{2} .
$$

The meaning of each term is exactly the same as in the text. If one assumes that $\underline{\hat{\xi}}$ and $\omega$ correspond to an unstable mode the terms involving initial conditions vanish, which yields $(\underline{\xi} \equiv \underline{\underline{\xi}})=0$. Finally, the dispersion functional reads as

$$
2 \delta \omega+i \omega \frac{e}{C} \int d \underline{x} n \hat{\xi}^{\star} \cdot \hat{\xi} \times \underline{B}_{0}+\omega \int d B \frac{S(B)}{B-\omega}=0 .
$$




\section{REFERENCES (CHAP. IV)}

1. R. Gerwin, Los Alamos National Laboratory Informal Report No. LA-6130-MS, (1975).

2. L. D. Pearlstein and J. L. Freidberg, Phys. Fluids 21, 1218 (1978).

3. J. P. Freidberg, Phys. Fluids 15, 1102 (1972).

4. H. R. Lewis and K. R. Symon, J. Math. Phys. 20, 413 (1979).

5. C. E. Seyler, Phys. Fluids 22, 2324 (1979).

6. K. R. Symon, C. E. Seyler and H. R. Lewis, J. Plasma Phys. 27, paper I, II and III, (1982), pp. 13-53.

7. C. E. Seyler and D. C. Barnes, Phys. Fluids 24, 1989 (1981).

8. M. D. Kruskal and C. R. Oberman, Phys. Fluids 1, 275 (1958).

9. R. M. Kulsrud, Phys. Fluids 5, 137 (1962).

10. J. M. Finn, Plasma Phys. 21, 405 (1979).

11. J. M. Finn and R. N. Sudan, Phys. Fluids 22, 1148 (1979).

12. J. M. Finn and R. N. Sudan, Nucleár Fusion 22, 1443 (1982).

13. J. L. Schwarzmeier and K. R. Symon, J. Math, Phys. 20, 979 (1979).

14. K. R. Symon, J. H lasma Phys. 29, 275 (i98j). 


\section{FIGURE CAPTIONS}

Fig. 4.1 Dynamical spectral densities and the Nyquist diagrams. Plot (a) is a dynamical spectral density of a travelling wave trial function. Plot (b) shows three Nyquist diagrams for $\gamma^{2}=1.0$, $r^{2}=.36$ and $r^{2}=0.0$ respectively. A11 the diagrams merge at a point on the real axis as $\sigma \rightarrow \pm \infty$. 

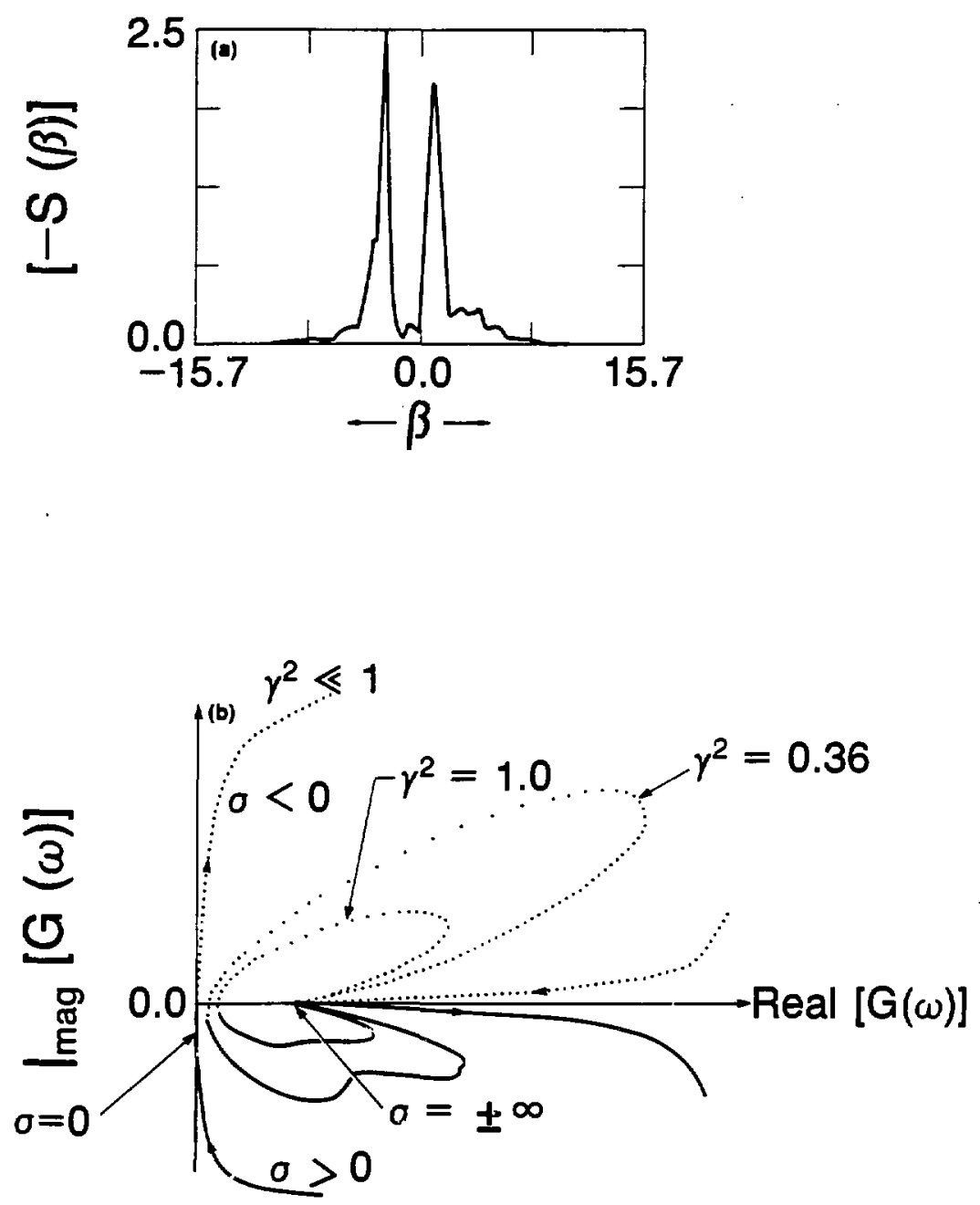


\section{CHAPTER $\checkmark$}

\section{THE STABILITY OF FIELD REVERSED CONFIGURATIONS (FRC)}

\section{A. Introduction}

The field reversed configuration (FRC) is an axisymmetric compact toroid characterized by the poloidal magnetic field only.

The FRC has some major advantages for a potential application to magnetic fusion; (1) It has high volume-averaged $8(.5<8<1.0)$, inside the separatrix (where $B$ is the ratio of local plasma pressure to external field pressure), since the plasma contained within the FRC separatrix is confined by closed magnetic field lines generated by toroidal plasma currents. (2) It has less engineering complexity than systems with toroidal symmetry due to magnetic coils, vacuum chamber and blanket. (3) Furthermore the field geometry possesses intrinsic divertor action and allows the plasma toroid to be translated along a guide field, which allows greater flexibility in reactor design.

Experimentaliy the plasmas of FRCS at LANL are found to be stable up to $40 \mu \mathrm{sec}$ for $F R X-B^{1}$ and $-100 \mu \mathrm{sec}$ for $F R X-C^{2}$ where they are terminated due to the rotational instability $(n=2$ mode where $n$ is the toraidal mode number). (With a quadrupole field the $n=2$ rotational instability is suppressed ${ }^{3}$ and the plasmas last longer). On the nther hand, ideal MHD and other present fluid calculations predict a set of very unstable modes ${ }^{4-10}(m \times 1$ shifting modes where $m$ is the poloidal mode number).

Study of ideal MHD $s$ in the neighborhood of the null line of a Hill's vortex indicates that $m=1$ modes are unstable for $n>1$ and 
becomes more unstable as $n$ increases. ${ }^{4}$ In the limit of large $n$ the modes are lccalized on a flux surface and simple analytic form of a dispersion relation can be obtained, which shows that the exponential growth time is the Alfvén transit time around a closed field line. If we take the parameters of FRCs at LANL, the growth times are around one usec. Even for lower $n$ modes, the growth rates are found to be of the same order. 5

Various other MHD equilibria ${ }^{6}$ (e.g., recetrack shape) have been considered but the result is no better. ${ }^{5}$ It is worth noting that a necessary condition for ideal MHD stability of a closed field line configuration is that the average of $\mathrm{krB}$ on the field line is positive where $k$ is the curvature of the magnetic field. ${ }^{7}$ Hence, shaping a closed line configuration will not convert the unstable modes to stable ones. However, it may affect the growth rates.

Many other MHD calculations have been attempted. Resistive effects $^{3}$ have been added to ideal MHD, but the effect turned out to be negligible on an Alfven time scale. The possibility of nonlinear saturation ${ }^{9,10}$ has also been investigated. Both the non linear energy variational calculation ${ }^{9}$ and the simulation result of the 3-D Malice code $^{10}$ show that the amplitude of fast growing modes do not saturate at a low level.

Thus a kinetic treatment seems to be necessary for the FRC stability against $n>1$ shifting $(m=1)$ modes. Especially since most ion orbits are big $(\sim 1 / 3$ of the size of the plasma) kinetic effects are very important even for global modes (low $n$ modes), which we will discuss in later chapters. 
In this chapter, we first investigate the linearized ideal MHD results and then other fluid calculations. The FRC experiments at LANL are briefly sumarized in Appendix $B$ to this chapter.

\section{B. Linearized Ideal MHD Theory}

\section{B.1 Near the Hill's Vortex}

The curvature driven ideal MHD instability in the neighborhood of the Hill's vortex of a FRC was studied by Newcomb ${ }^{4}$ for arbitrary elongated flux surfaces. The word 'neighborhood' means the region where the Taylor expansion of the flux function is valid. Near the magnetic field null (' $O$ ' point) the local $B$, ratio of plasma pressure to magnetic field pressure, becomes very large. If we restrict frequency ranges to the order of $\Omega_{A}$, where $\Omega_{A}$ is the angular Alfven velocity (i.e. $2 \pi / \Omega_{A}$ is the time required for one complete transversal of a closed magnetic flux line by an Alfven-wave signal), $\underline{\nabla} \cdot \underline{\xi}=0$ is a good approximation in the high $B$ limit since $\underline{\nabla} \cdot \underline{\xi}$ is order of $B^{-1}$.

In general the potential energy variation can be expressed in terms of three terms; surface term, vacuum term and fluid term. Since the displacements near the nu1l are internal, the surface term vanishes. Also, the internal modes do not affect the vacuum fields, thus the vacuum term vanishes. For an incompressible displacement, the fluid part of the potential energy variation is from Ref. 4 [Newcomb]. 


$$
\delta W(\underline{\xi}, \underline{\xi})=\int \underline{d x}\left\{\underline{\xi^{*}} \underline{\underline{\xi}}: \underline{\nabla}\left(\rho_{0}+\frac{B_{0}^{2}}{8 \pi}\right)+\frac{1}{4 \pi}\left|\underline{B}_{0} \cdot \nabla \underline{\underline{\xi}}\right|^{2}\right\}
$$

where $B_{0}$ and $P_{0}$ are the equilibrium magnetic field and the pressure. The first term is the pressure driven destabilizing term and the second is the stabilizing magnetic bending energy.

For an axis symmetric toroidal system it is sucgestive to do the Fourier transformacion of a displacement vector $\underline{\xi}$ with respect to the toroidal angle $\theta$. Upon inte!!rating over $\theta$, a reduced energy variational is obtained. In terris of the flux coordinates $\psi$ and $x$ where $\psi$ is the flux function and $x$ is the coordini":e along the magnetic field, the energy variationl can be expressed as

$$
\delta W\left(\xi_{n}{ }^{\star}, \xi_{n}\right)-\int d \psi d x\left\{-\left(\xi_{x}{ }^{2}+\xi_{y}{ }^{2}\right)+b\left(\xi_{x, x^{2}}+\xi_{y, x}^{2}+\frac{1}{n^{2}} \frac{\xi_{z, x}^{2}}{2}\right)\right\}
$$

where $\xi_{i, x}$ means the derivative of $\xi_{j}$ with respect to $x$ and $b$ is a constant. Since the integrand of expression (5.2) does not involve any derivatives with respect to $\psi$, sW can be minimized on each flux surface $(\psi=$ constant). Notice that the mode number $n$ appears in only one positive term as $1 / n^{2}$. Therefore s $w$ is minimized by taking $n \rightarrow \infty$ and it is sufficient to show $s W<0$ for $n \rightarrow \infty$ to prove instability. To estimate the growth rates of a mode, a variational formulation is useful. Minimizing the potential energy variation, with respect to a normalization $K\left(\xi^{*}, \xi\right)=\frac{1}{2} \int \rho \xi^{\star} \cdot \xi d \underline{d}$, which is always positive, we have from the self adjointness of the operator in $\delta h$,

$$
\omega^{2} \equiv \delta W / K(\underline{\xi}, \underline{\xi}) .
$$


For unstable modes $\delta \mathrm{W}$ is more negative as $n$ increases, minimizing the stabilizing magnetic bending energy, and $K$ becomes small. Therefore, it is clear that the mode becomes more unstable as $n$ increases. So we want to evaluate the growth rates in the limit of $n \rightarrow \infty$. In this limit, modes are highly localized and so each flux surface can be treated independently. Furthermore, the Euler-Lagrange equations for radial and axial displacements obtained from the Lagrangian $L=\delta W-K\left(\dot{\xi}^{*}, \dot{\xi}\right)$ are decoupled and identical, thus yielding the same spectrum of eigenfunctions.

The unstable axial mode is a rigid shift displacement in the axia? direction (each flux surface moves rigidly in the axial direction), and the same for the radial mode in the radial direction. For both modes the growth rates are $\varsigma_{A}$, or tiie e-folding growth time is the Alfven transit time around the closed magnetic flux lines.

Since the axial and radial modes are degenerate they are equally unstable for any elongated flux surfaces in the neighborhood of the null where the density is approximated to be uniform. This is no longer true if the mode is localized auya from the null where the density gradient should be taken min account as one might expeci since the radial displacement requires more magnetic field compress ion than that of the axial mode. If we use cylindrical coordinates rather than Cartesian coordinates the above mentioned rigid shift modes correspond to the $m=1$ poloidal mode. Now the degeneracy is in the sign of $m$. The case of $m=-1$ is equivalent to that of $m=1$. The modes corresponding to $m=0$ and 2 are marginally stable and those corresponding to $m>2$ are stable. 
It is worth mentioning that the most unstable mode is not the: interchange mode $(m=0)$ but the rigid shifting mode $(m=1)$. Tin's is because there is no magnetic bending in the rigid shifting case. Hence the most unstable MHO mode is $m=1$ (no magnetic bending energy) and $n \rightarrow \infty$ (smallest magrietic compression). Detailed discli_-_im on the interchange mode is given in a later section.

\section{B.2 St:abi; ty of a Hill's Vortex}

Now we study more serieral cases, drapping the incompressibility assumption, thus the calcuistion is valid somewhat away from tine null. All the rest of the conditions are considered to be the same as before. Therefore following the same argidment described earlier, we only have to consider the limiting case of $n \rightarrow \infty$. In this limit sW can he mininized with respect to $\xi_{\theta}$ and $\xi_{X} \equiv \xi_{\xi^{*}} \underline{B}_{0}$ and the variation involves only $\xi_{\psi}$ where $\psi$ is the flux function. The final expression of $\delta W$ does not involve derivatives with respect to $\psi$, sW can be minimized on each flux surface (localization of the mode) which leads to an integral differential equation for $\xi_{\psi}$ with respect to $x$ [see Ref. 11 and 12]. By solving the equation numerically for various elongation of the flux surfaces, Finn has found three unstable modes in general of a Hill's vortex equilibrium in the limit of large aspect rativ. They are lowest even mode (interchange), second even mode and the first odd mode (Ballooning mode) with respect to where the poloidal angle 6 is measured from the minor axis of an elliptical flux. Of course, for circular flux surfaces $(b / a=1$ where $b$ and $a$ are the major and the 
minor radius of the flux surface) the second even and the ballooning modes are degenerate.

If one chooses a pressure profile directly proportional to the flux function, the interchange mode becomes more unstable as the flux surface becomes more elongated up to $b / a \sim 2$, but then it is stabilized if the elongation is strong ( $b / a>3)$. The stability of an interchange mode is rather sensitive to the pressure profile and will be discussed later in detail. However, it is worth pointing out that the mode can be stabilized by tailoring the pressure profile.

The second even mode and the ballooning mode are unstable for any elliptical or circular flux surfaces. As the elongation increases, the second even mode becomes less unstable and approaches to marginal stability in the limit of a slab geometry, but the ballooning mode becomes more unstable. Due to the normalization condition, Finn's work ${ }^{11}$ doesn't show the growth rates quantitatively but it shows clearly that the most dangerous mode is the ballooning mode for an elongated flux surface.

\section{B.3 Interchange mode $(m=0)$}

The plasmas confined by the closed-line configuration have unfavorable curvature to interchange modes. On the other hand the . magnetic gradient drift (i.e., the gradient of the magnetic field) is favorable everywhere along the closed lines in field reversed systems. It is found that flutetype perturbations in a low a plasma confined by closed field lines are unstable unless the current profile is nollow. 13 
Sparks, Finn and Sudan ${ }^{14}$ have studied the interchange stability of high s plasma of an axis-symmetric field reversed equilibrium by solving the Grad-Shafranov equation for the equilibrium and varying the prossure on the magnetic axis and the pressure on the separatrix independently. The stability criterion against the interchange is that $V^{\prime \prime} / V^{\prime}+\left(P^{\prime} / \gamma P>0\right.$ or $\left(P V^{\prime}\right)^{\prime}>0$ where $V^{\prime}=2 \pi f \frac{d]}{B}$ and the prime indicates the derivative with respect to the flux function $\psi$. It is not too difficult to obtain a reasonable pressure profile to meet the stability condition inside the separatrix. Outside the separatrix the curvature itself is favorable to stability and so the field lines in the region are stable. The field lines near the separatrix can be made stable when the pressure at the separatrix is finite. The requirement of finiteness of the pressure at the separatrix could be satisfied by making the pressure essentially zero for some equilibria. For example if $v^{\prime} \sim-\ln |\psi|$, then the pressure at the separatrix $(\psi=0)$ could be made as small as $(-\ln |\psi|)^{-\gamma}$ where $\gamma$ is the ratio of specific heat capacity of constant pressure to that of constant volume.

\section{B.4 Rigid Axial Displacement Modes ( $m=1 ; n=0,1, \ldots$ )}

B.4a. Roman Candle Mode $(n=0)$

$n=0$ mode is an axial displacement of the entire flux tubes. Without strong mirrors at the ends this mode can be dangerous: The so-called "roman candle" mode, results in an ejection" 15 , of the 
entire plasma out the ends of the device. It has been shown by using the full ow expression for the $n=0$ mode that $P^{\prime \prime}(\psi)<0$ is sufficient for stabilizing the mode. ${ }^{16}$ Therefore, like the interchange mode, a proper pressure profile makes the plasma stable with respect to the "roman candle" mode.

\section{B.4b. Tilting Mode $(n=1)$}

The tilting mode has been one of the most important modes for many years in a compact toroidal system such as spheromak, FRM and FRC. The reason is that the mode is a global mode and predicted to be very dangerous.

The tilting mode is predicted ${ }^{17}$ and observed ${ }^{18}$ in a spheramak but can be stabilized by the wall. However the mode has never been observed in FRC's.

The general fluid properties of the tilting mode in FRC's has been already discussed in the previous sections. Therefore we focus here on a detailed structure of the mode. Schwarzmeier et al. ${ }^{5}$ have examined the MHD tilting instability both by an initial value code and by a "semi"-trial function (explained below) approach for various FRC configurations ranging from elliptical to highiy racetrack shaped equilibria.

The time dependent equations are obtained by linearizing the resistive MHD equations about an equilibrium. The perturbations are assumed to vary as $f_{1}(r, z, t) e^{\text {ine }}$ where $\theta$ is the toroidal angle. The unstable equilibria result in exponentially growing solutions. The result of the initial value code runs can be summarized as follows: 
(1) For elliptical equilibria, each flux surface has a rigid axial displacement i.e., $\underline{\xi}(\psi, x)$ where is the coordinate along the magnetic field line with maximum displacement near the null. (2) As the equilibria become more flattened like a racetrack, the displacements are more localized near the tips of the flux surfaces, indicating a strong dependence upon $x$. (3) The growth rates are insensitive to the flatness of the equilibria. The growth times of the four different equilibria are about one $\mu$ Sec for the parameters of a $F R X-B$.

The growth rates of the modes are also investigated by a semitrial function approach. The word 'semi' is used because the form of a trial function is chosen and then the detailed structure of the mode and the growth rates are evaluated by solving a dispersion matrix introduced in Chap. IV. Specifically, the trial function is chosen such that it is incompressible $(\underline{\nabla} \cdot \underline{\underline{G}}=0)$ and is a rigid motion $(\underline{\xi}(\psi, x) \equiv \xi(\psi) \hat{c}$ where $\hat{c}$ is a constant vector in the $(r, z)$ plane $)$. Now $\xi(\psi)$ is expanded in terms of basis functions $n$ as

$$
\xi(\psi ; w)=\sum_{\ell} a_{\ell}(w) \eta_{\ell}(\psi)
$$

By this procedure, Schwarzmeier et al. found a very good agreement, within $10 \%$, on the growth rates with che initia? value code result for an elliptical flux surface. However, the growth rates are very much reduced (growth time $20 \mathrm{\mu sec}$ ) for highly racetrack shaped equilibria, showing a big discrepancy with the inital value code result. The $x$ reason is that the basis functions chosen do not allow $x$ dependence of 
the mode. The work indicates that (1) the trial functions should be taken carefully and (2) the rigid displacement assumption is good for an elliptical equilibria.

B.4c. Ballooning Modes $(m=1, n \rightarrow \infty)$

The curvature of the magnetic field in a FRC is bad everywhere within the separatrix with strong curvature near the tips of the flux surfaces. Hence a perturbation which is not constant but varies slowly along the field line $\left(k_{\|} \ll k_{\perp}\right)$ can develop in such a way that the mode is concentrated in the most unfavorable region, which results in a stronger pressure-driven destabilizing effect than that of a simple interchange mode. If the concentration is not too severe, the increase of stabilizing force from the line bending cannot overcome the pressure-driven destabilizing effect.

In general ballooning modes are difficult to solve because of the inherent multidimensional property. However in the limit of large $n$, modes are localized on each flux surfaces $\left(k_{\perp} L_{\text {. }} \gg 1\right)$, in which case the stability problem reduces to a 1-D differential equation on the surface. When such a calculation is applied to a FRC configuration, ballooning modes are found to be the most unstable.

However, they are presumably stabilized easily by finite orbit effects, since the modes are localized. 


\section{Other Non-kinetic Theories}

\section{C.1 Double Adiabatic}

A more realistic model than ideal MHD is the double adiabatic theory. This model assumes that the magnetic field is strong enough for particles to gyrate about the lines (no heat flow across the field) and while the temperature is uniform along the line of force where heat can flow easily. This assumption leads to two adiabatic equations of state

$$
\frac{d}{d t}\left(\frac{P_{\perp}^{2} P_{\|}}{\rho^{5}}\right)=0 \text { and } \frac{d}{d t}\left(\frac{P_{1}}{\rho B}\right)=0 \text {. }
$$

Where $P_{\perp}$ and $P_{\| \mid}$are the pressure perpendicular and the pressure parallel to the magnetic field lines respectively. The second equation of state is equivalent to the adiabatic ${ }^{t} y$ of magnetic moment. These equations of state form a closed set of $e_{4}$ ations with the continuity equation, the momentum equation, $\underline{E}+\underline{u} \times \underline{B}=0$ and Maxwell's equations and are known as the CGL equations or double adiabatic equations.

First we assume that the unperturbed pressure is isotropic i.e., $P_{\perp}{ }^{0}=P_{\|}{ }^{0}=P^{0}$. Somewhat tedious but straightforward calculation ${ }^{19}$ shows that the normal mode frequencies of a circular flux surface near the null in the limit of $n \rightarrow \infty$ are

$$
\omega^{2}=\frac{5}{2}\left(m^{2}+1\right) \frac{p_{0}}{\rho_{0} r^{2}} \quad \text { and } \omega^{2}=\frac{m^{2}\left(m^{2}-3\right)}{m^{2}+1} \frac{B_{0}{ }^{2}}{\rho_{0} r^{2}}
$$


where $\rho_{0}$ is the equilibrium density on the flux surface and $r$ is the radius of the surface. The first dispersion relation corresponds to sound waves which are stable for all poloidal mode numbers. The second dispersion relation indicates the instability of the $m=1$ mode with exactly the same growth rate as that of ideal MHD. The reason that we have the same growth rates as idea 7 MHD for the $m=1$ mode is that the mode is essentialiy a rigid displacement of a single flux surface (the mode is localizer as $n \rightarrow \infty)$ and the role of double adiabaticity is minimized.

Grossmann, Hameiri and Weitzner 20 investigated the double adiabatic stability of Compact Torus plasmas without the isotropic restriction on $P_{0}$ for variously elongated flux profiles. They found some evidence of the stabilizing effect near the nutl due to the pressure anisotropy for somewhat large $m$ transversal modes (perpendicular to the toroidal axis and the axial direction). The transversal mode is often called a radial mode. However, it is still premature to make a general conclusion. Furthermore the most unstable MHD modes of an elongated plasma are longitudinal (axial) not transversal.

\section{C.2 Resistive MHD}

Resistive effect to the shifting modes have been in'estigated. ${ }^{8}$ However for the fast growing modes, whose growth times are of the order of Alfven iransit times, the dispersion relations are found to be unaffected by the inclusion of the plasma resistivity. 


\section{C.3 Nonlinear Ideal MHD}

Nonlinear effect on stability have also been studied to see whether the amplitude of the lineariy unstable displacements saturate at a low level. A study ${ }^{9}$ has been made by finding a nonlinear energy variational of an adiabatic flow with infinity conductivity, which is the exact difference et ee the potential energies of the final and the initial configurations governed by ideal MHD for an arbitrary finite displacement. The result is that for an entire class of compact toroidal systems with no toroidal magnetic field deformation, corresponding to a tilting mode, there is growth even in the nonlinear regime (after having gone through the Tinear regime with a high growth rate).

\section{C.4 Racetrack Shaped Equilibria}

The experiments of FRC at LANL indicates that the equilibria are not exactly elliptical but somewhat flatened. Many possible equilibrium Field Reversed configurations are numerically obtained by solving the Grad-Shafranov equation. 6 "We know that choosing a different equilibrium of a closed field line system does not change the stability, because a necessary condition for stability is that the average of $k r B$ on a closed field line be positive where $k$ is the curvature of the magnetic field line. ${ }^{7}$ For closed field line systems of confined plasmas the condition is never satisfied. The necessary stability condition is obtained from the condition that the sign of energy variational should be positive for a rigid displacement in the elongated direction. 
Even though the racetrack equilibria do not cause the stability of the plasma it is interesting to know how sensitively the eigenmodes and the growth rates depend on the detailed shape of the equilibria. For interchange modes, the growth rates are sensitive to the pressure profile, in contrast to tilting modes which are insensitive to the equilibrium configuration. The eigenmodes vary from rigid shift of a flux surface for an elliptical equilibria to a highly localized mode near the tips of the equilibria for a racetrack-shaped equilibria. The growth rates are rather insensitive to the equilibrium configuration, all showing high growth rates. 5 


\section{APPENDIX B \\ FIELD REVERSED EXPERIMENTS (FRX) ON COMPACT TOROIDS ${ }^{21}$}

The field-reversed configuration (FRC) is an axis symetric highly prolate compact toroid formed with no toroidal magnetic field as shown in Fig. 6.1.

The formation of FRC is best described by the following four steps. (1) Preionization: an initial bias field is frozen into a cold preionized plasma in a cylindrical conducting wall. (The ratio of length to radius is about 8.) (2) Implosion: then the direction of the current in the coil surrounding the cylinder (the theta pinch coil) is quickly reversed by the main bank discharge. (3) The resulting oppositely directed fields tear and reconnect at the ends, generating a closed field line structure. (4) With a damped radial-oscillation the plasma contracts axially to form a well defined and quiescent FRC as shown in Fig. 6.1.

There are three FRX's at LANL; FRX-A, FRX-B and FRX-C. FRX-A is a low energy device which is only capable of operating over a narrow range of initial deuterium filling pressure $\left(P_{0}\right)$ while $F R X-B$ is more energetic device than FRX-A so that it can be operated over a wide range of $P_{0}$. The main difference between the two facilities is in the capacitor banks, especially in the main bank energies. FRX-C differs from FRX-B in its physical dimensions. It is about twice as large as FRX-B radially and axially and all the other parameters are almost the same as those of FRX-B. 
The magnitude of axial magnetic field is obtained from external magnetic probes located just outside the quartz tube on the axial mid plane. The main bank discharge creates a field, in the opposite direction with respect to the bias field, which rises up to $8 \mathrm{KG}$ and decays slowly to $5 \mathrm{KG}$ at the termination of the FRC pulse.

The plasma density and the temperature of electrons $\left(T_{e}\right)$ can be deduced from Thomson scattering data. Figure 6.2 shows a typical density and $T_{e}$ profile of a $F R X-B$ at $t=10 \mu-s e c$ after the main bank discharge. The plasma density is maximum at the magnetic null and the density is still significant at the separatrix. The electron temperature is found to be uniform spatially up to the separatrix and constant in time. The single measurement outside of the separatrix at $r-6 \mathrm{~cm}$ is less definite due to lower signal level and the greater shot to shot plasma fluctuations. Once we know $T_{e}$, the total plasma temperature, and thus $T_{j}$, can be estimated from pressure balance, $T_{e}+T_{j}=\langle\beta\rangle B^{2} / 8 \pi n$, where $\langle\beta\rangle$ is the volume averaged $\beta$ within the spearatrix and $B$ is the measured external magnetic field. Ion temperature and ion rotational velocity are measured by the Doppler profile of the $2271 \mathrm{~A}$ line of carbon $V$. These measurements provide a good estimate of $T_{i}$ for the $D^{+}$plasma but provides a poor estimate of the rotational velocity. Flux loops and field probe arrays are used to measure the excluded flux vs. axial position.

The typical experimental parameters of FRX-B and FRX-C are illustrated in Table I. The confinement time $\tau$ here is measured without the quadrupole field which removes the $n=2$ rotational instability. 


\section{REFERENCES AND FOCTNOTES (CHAP. $V$ )}

1. W. T. Armstrong, R. K. Linford, J. Lipson, D. A. Platts and E. G. Sherwood, Phys. Fluids 24, 2068 (1981).

2. K. F. Mckenna, W. T. Armstrong, R. R. Bartsch, R. E. Chrien, J. C. Cochrane, Jr., R. W. Kewish, Jr., P. Klingner, R. K. Linford, D. J. Rej, E. G. Sherwood, R. E. Siemon, and M. Tuszewski, Phys. Rev. Lett. 50, 1787 (1983).

3. S. Ohi, T. Minato, Y. Kawakami, M. Tanjyo, S. Okada, Y. Ito, M. Kako, S. Gotô, T. Ishimura, and H. Itô, Phys. Rev. Lett. $\underline{51}$, 1042 (1983).

4. W. Newcomb, Phys. Fluids 23, 2296 (1980).

5. J. L. Schwarzmeier, D. C. Barnes, D. W. Hewett, C. E. Seyler, A. I. Shestakov and R. L. Spencer, Phys. Fluids 26, 1295 (1983).

6. D. W. Hewett and R. L. Spencer, Phys. Fluids 26, 1299 (1983).

7. J. R. Cary, Phys. Fluids 24, 1818 (1981).

8. A. I. Shestakov, J. Killeen, and D. D. Schnack, J. Comp. Phys. 46, 69 (1982).

9. Yu A. Dreizin and E. P. Sokolov, Sov. J. Plasma Phys. 2, 516 (1981).

10. D. C. Barnes, A. Y. Aydemir, D. V. Anderson, A. Z. Shestakov and D. D. Schnack, "Proceerings of the 3rd Symposium on Physics and Technology of Compact Toroids of the Magnetic Fusion Energy Program" Los Alamos Report No. LA8700-C, Los Alamos, NM, 2-4 Dec. 1980 . 
11. J. M. Finn, Phys. Fluids 24, 274 (1981).

12. I. B. Bernstein, E. A. Frieman, M. D. Kruskal and R. M. Kulsrud, Proc. R. Soc. London Ser. A 244, 17 (1958).

13. G. Benfard, D. L. Book, N. C. Christofilos, T. K. Fowler, V. K. Neil and L. D. Pearlstein, in Plasma Physics and Controlled Nuclear Fusion Research (IAEA, Vienna, 1969) Vol. I, p. 981.

14. L. Sparks, J. M. Finn and R. N. Sudan, Phys. Fluids 23, 611 (1980).

15. C. E. Seyler, W. Grossmann and L. C. Steinhauer, Comments on Plasma Physics, 4,21 (1978).

16. D. C. Barnes and C. E. Seyler, Los Alamos National Laboratory Report No. LA-UR-79-13 (1979).

17. M. N. Rosenbluth and M. N. Bussac, Nucl. Fusion 19, 489 (1979).

18. T. R. Jarboe, I. Henins, H. W. Hoida, R. K. Linford, J. Marshall, D. A. Platts, and A. R. Sherwood, Phys. Rev. Lett. 45, 1264 (1980).

19 J. H. Hammer private communication.

20. W. Grossmann, E. Hameiri and H. Weitzner, Phys. Fluids 26, 508 (1983). 
Table 1. Experimental parameters of Field Reversed Experiments at LANL.

\begin{tabular}{|c|c|c|}
\hline Parameters & $5 \mathrm{R} X-\mathrm{B}$ & FRX-C \\
\hline$L_{c}(\mathrm{~cm})$ & 100 & 200 \\
\hline$r_{w}(\mathrm{~cm})$ & 12.5 & 25 \\
\hline$r_{s}(\mathrm{~cm})$ & 5 & 10 \\
\hline$=a / b$ & $\because .1$ & -.1 \\
\hline$n\left(\mathrm{~cm}^{-3}\right)$ & $(1-4) \times 10^{15}$ & $(1-4) \times 10^{15}$ \\
\hline$T_{e}(e V)$ & $100-200$ & $100-200$ \\
\hline$T_{i}(e V)$ & $100 \sim 1000$ & $100 \sim 1000$ \\
\hline Bext & $6-8$ & $6-8$ \\
\hline$\tau($ usec $)$ & 40 & 100 \\
\hline
\end{tabular}




\section{FIGURE CAPTIONS}

Fig. 5.1 Field Reversed Configurations. The characteristic dimensions of FRC's at LANL are $a / b-.1$ and $r_{s}-\sqrt{2} R$. All parameters are shown in ble 1 .

Fig. 5.2 Typical density and electron temperature profile of a FRX-B at LANL at about 10 usec after the main bank discharge. The data are taken from Ref. 1. 


\section{Conducting wall}

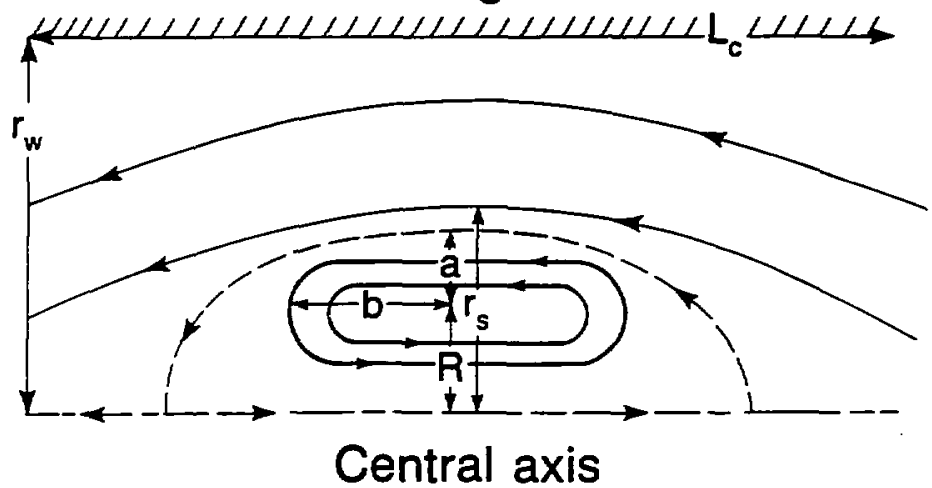

Figure 5.1

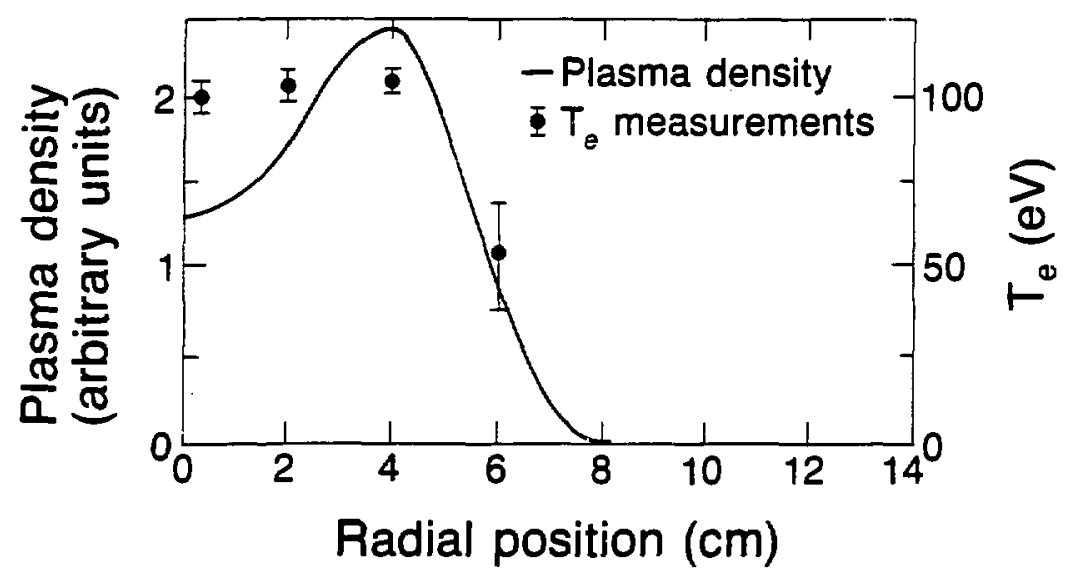

XBL 8311.7345

Figure 5.2 


\section{CHAPTER VI \\ VLASOV FLUID (VF) STABILITY ${ }^{1}$ ANALYSIS OF AN ELONGATED Z-PINCH}

\section{A. Introduction}

As is described in previous sections, the stability of axialshifting modes of a Field Reversed Configuration (FRC) requires more sophisticated analysis than MHD. In this work we investigate the kinetic effects on these displacements by approximating a FRC by an elongated Z-pinch.

The cylindrical approximation of a Compact Torus sounds like a bad approximation because of the small aspect ratio. Nevertheless, MHD theories predict the same instabilities of shifting modes in an elongated Z-pinch as in FRC. This is because there is no magnetic bending involved in making a toroidal configuration from a cylindrical shape since there is no toroidal field in a FRC. Furthermore the elongated Z-pinch has other essential properties of a FRC such as a field null, closed field lines, large particle orbits and particle stochasticity. The centrifugal force of a toroidal system may play an important role, but our concern for the moment is to find the major kinetic corrections to MHD stability.

We investigate the kinetic effects by adopting a VF model. 1 The electrons in the FRC experiments at LANL ${ }^{2,3}$ are collisional while ions are somewhat collisionless in an MHD time scaling. The temperature of the electrons is usually lower than that of ions. We approximate the electrons as a cold pressureless fluid and the ions as collisionless. 
A VF equilibrium is found following the procedure described in Chap. IV. As a VF equilibrium the magnetic field configuration of the z-pinch is exactly the same as the one used in the orbit study in Chap. III. However, in general an electric field is present.

The orbits in this VF equilibrium can be understood by simply studying the effects of the electric field. The main effects are that (1) ions are confined electrostatically and (2) measure of stochastic orbits in the system with $\underline{E}_{0}=0$ is significantly reduced. Other than these factors, the character of the orbits is essentially the same. As is the case when $\underline{E}_{0}=0$, if we take the FRX parameters at LANL we find that more particles are stochastic as the size of the plasma becomes large and more particles are regular as the temperature of ions increases. At any event the $\mu$-conserving orbits are negligible where $\mu$ is the magnetic moment. If we apply our orbit results to the experimental parameters, we find that the ion orbit system ranges from very stochastic to very regular. This suggests that the stochasticity is not so important as the stabilizing effect of the shifting modes as much as large orbits are, even though the stochasticity plays a crucial role in some modes.

Treatment of large orbits, not to mention the stochastic ones, is in an extremely primitive stage. To explain the stability of FRC we need to employ a method which deals with both the regular and the stochastic orbits. The term which needs the orbit information in the VF dispersion functional can be evaluated both for regular and stochastic orbits by evaluating correlations and the dynamical spectral densities numerically for a chosen trial function. 
The exact dispersion relation of the VF model can be obtained by solving a matrix which is obtained by evaluating the spectral densities for a set of basis functions. However, our tentative purpose is to develop a method of treating the large orbits including both the regular and the stochastic and test the feasibility of using the technique with a matrix problem. By choosing a set of ideal MHD eigenvectors as the trial functions, we find the primary effect of VF corrections to MHD.

Analytical formulae of the trial functions are used rather than the exact eigenmodes of ideal MHD in order to reduce the computational work. Due to the peculiarity of the standing wave trial functions we express the displacements in the form of a traveling wave in $z$. To study the null effect two types of radial dependencies are considered: one has large displacement near the null and the other has large displacement away from the null.

\section{B. A VF Equilibrium in a Z-pinch}

Taking the parameters of the FRC at LANL, we obtain the electron collision time as $-10^{-8} \mathrm{sec}$ and ion collision time as $10^{-5} \mathrm{sec}$. Therefore on an MHD time scale $\left(-10^{-6} \mathrm{sec}\right)$ electrons can be considered as collisianal while ions are treated as collisionless. For simplicity, we neglect the electron pressure, in which case the VF model is suitable to investigate the kinetic stability of FRC's at LANL. In this model electrons are governed by $\underline{E}+\frac{1}{C} \underline{u}_{e} \times \underline{B}=0$ and ions by the Vhasov equation. Since the detailed picture of the model is described 
in Chap. IV, we proceed to find a VF equilibrium of an elongated Z-pinch.

If we further assume that the equilibrium ion distribution is a function of energy only,

$$
f_{0}(\underline{x}, \underline{v})=F_{0}(H(\underline{x}, \underline{v}))
$$

where $H=\frac{1}{2} M v^{2}+e \phi_{0}(\underline{x})$ and

$$
v_{z}=\frac{1}{M}\left(P_{z}-\frac{e A_{0}}{C}\right)
$$

Here $\underline{A}_{0}$ is the equilibrium vector potential and $\underline{A}_{0}=A_{0} \hat{z}$. As is shown in Chap. IV, the force balance equation of a VF model for a class of ion distributions of Eq. (6.1) is

$$
\frac{1}{C} \underline{J}_{0} \times \underline{B}_{0}=n_{0} e \underline{E}_{0}=\underline{\nabla P}_{0},
$$

which is the same as that of ideal MHD. Thus the equilibrium flux configurations can be obtained by inverting the operator

$$
-\nabla^{2} \psi=4 \pi \frac{d P(\psi)}{d \psi}
$$

to find $\psi$. The stream function $\psi$ is exactly $A_{0}$ in a cylindrical system and so

$$
\underline{B}=\frac{\partial \psi}{\partial y} \hat{x}-\frac{\partial \psi}{\partial x} \hat{y} \quad \text {. }
$$


The pressure $P(\psi)$ of $\mathrm{Eq} .(6.3)$ is a free function, which should of course satisfy the boundary conditions, and can be specified as close as possible to the experiment.

The density profile of FRC is maximum at the null and decreases monotonically away from the null (see Fig. 5.2). The density at the separatrix is rather significant. We approximate the density profile as a quadratic function in $x$ and $y$ :

$$
n_{0}(b)=n_{0}(0)\left(1-\varepsilon_{d} \phi\right)
$$

where $b=\varepsilon^{2} x^{2}+y^{2}$ and $c_{d}$ parametrizes the relative pressure at the "pseudo" separatrix to that at the null. The word "pseucio" is used since in our Z-pinch model there is no actual separatrix. Nevertheless since our model is intended to describe the toraidal FRC, it is convenient to use the word separatrix for the flux surface corresponding to the real separatrix of a FRC. From now on we drop the word pseudo. The ions are confined electrostatically in the region of $0<b<\mathrm{ed}^{-1}$. The density profile of Eq. (6.5) does not vanish smoothly at the edge of the plasma and so the potential approaches infinity as $b \rightarrow c_{d}^{-1}$. Therefore, the equilibrium configuration of our Z-pinch is not realistic near the plasma boundary. However, we are interested in the internal modes which vanish at the separatrix; the configuration outside of the separatrix is presumably not important. The experiments show that the temperature of electrons and ions are fairly uniform throughout the plasma. Thus 


$$
\underline{\nabla} P_{0}=T \underline{\nabla} n_{0}
$$

From Eqs. (6.3) and (6.6) we obtain a class of equilibria which is similar to the Hill's vortex of a torus

$$
\psi=\frac{B}{2 a} b \quad, \quad \text { where } \quad b=\varepsilon^{2} x^{2}+y^{2},
$$

which describes an elliptical Z-pinch. Here a is the minor radius of the separatrix, $B_{a}$ is the magnetic field strength at $x=0$ and $y=a$ and $\varepsilon$ parameterizes the elongation of the flux contours.

Once we obtain the equilibrium flux contours, all the other equilibrium quantities are obtained easily. From Eq. (6.4) the magnetic field is

$$
\underline{B}_{0}=\frac{B}{a}\left(y \hat{x}-\varepsilon^{2} x \hat{y}\right)
$$

and from Eq. (6.2) the electric field is

$$
\underline{E}_{0}=\frac{2 T \varepsilon_{d}\left(\varepsilon^{2} x \hat{x}+y \hat{y}\right)}{e\left(1-\varepsilon_{d} \phi\right)}
$$

The relation between the density gradient and the magnetic field gradient is

$$
\varepsilon_{d}=\frac{\varepsilon^{2}+1}{2 \pi n_{0}(0) T}\left(\frac{B}{2 a}\right)^{2}
$$


The electrostatic potential is easily evaluated from the expression of Eq. (6.9)

$$
\Phi_{0}(\phi)=-\frac{T}{e} \ln \frac{n_{0}(b)}{n_{0}(0)}=-\frac{T}{e} \ln \left(1-\varepsilon_{d} b\right)
$$

where we have chosen $\Phi_{0}(0)=0$.

Some of the properties of the equilibria are as follows: (1) $B_{0}$ is tangent to the flux surfaces and $E_{0}$ is perpendicular to them. (2) On a single elliptical flux surface, $\underline{B}_{0}$ is weak at the tips of the ellipses and so is $\underline{E}_{0}$. (3) $\underline{B}_{0}$ increases linearly with the distance of the flux surfaces from the null, but $\underline{E}_{0}$ increases faster than linearly and eventually diverges at $\phi=\varepsilon_{d}^{-1}$ where the density vanishes.

\section{Character of Ion Orbits in the Z-pinch}

In Chap. III, we have thoroughly studied the ion orbits in an elongated Z-pinch whose flux function is the same as Eq. (6.7) with no equilibrium electric field. ${ }^{4}$ There remains the task of studying the orbits in the VF equilibrium with an electric field present. We need to determine the behavior of orbits and how much the population of each type of orbit of a confined plasma will be affected.

First let us summarize the results of Chap. III. Roughly speaking, there are three types of motion near a linear magnetic field null wher: there is no electric field: (1) $J_{y}$-conserving regular orbits stochastic orbits and (3) $u$-conserving regular orbits, where $J_{y}$ is the action in $y$ which is the coordinate in the minor axis of the flux 
surface and $w$ is the magnetic moment. The $J_{y}$ conserving orbits are composed of two types of motion; the figure eight type and the betatron type. Let $z$ be the axial direction. Orbits with $P_{z}<0$ are mostly regular with $J_{y}$ as an invariant. They are all betatron type. Orbits with $P_{Z}>0$ show complicated behavior. The behavior of orbits can be best characterized by the ratio of energy of the particle to the local peak potential energy at the field null, $\mathrm{P}_{\mathrm{z}}{ }^{2} / 2 \mathrm{M}$. If $\mathrm{H}>\mathrm{P}_{\mathrm{z}}{ }^{2} / 2 \mathrm{M}$, orbits are figure 8 type $J_{y}$-conserving and if $\mathrm{H}<.04 \varepsilon^{3}\left(P_{z}{ }^{2} / 2 M\right)$ orbits are $\mu$-conserving otherwise stochastic. If we take the parameter of $\varepsilon=0.1$ from the FRX at LANL, the $\mu$ conserving orbits occur on $1 y$ when $H<4 \times 10^{-5}\left(P_{z}^{2} / 2 M\right)$.

To study the electric field effect, we write down the Hamiltonian $H$ of an ion

$$
H=\frac{P_{x}^{2}}{2 M}+\frac{P_{y}^{2}}{2 M}+\frac{1}{2 M}\left(P_{z}-\alpha \phi\right)^{2}-T \ln \left(1-\varepsilon_{d} \phi\right)
$$

where

$$
a \frac{e B a}{2 c a}
$$

and where the effective potential $U$ in $x y$ plane is

$$
U=\frac{1}{2 M}\left(P_{2}-\alpha b\right)^{2}-T \ln \left(1-\varepsilon_{d} \phi\right) \quad .
$$

The additional term, the second term of Eq. (6.13), due to the electric field is, zero at the null and increases gradually near the null, but approaches infinity as $b \rightarrow \varepsilon_{d}^{-1}$. The effective potentials of the two 
cases are very much the same for small values of $P_{z}\left(\alpha^{-1} \varepsilon_{d} P_{z} \ll 1\right)$ which suggests that the criterion of regular and stochastic orbits is approximately the same as in the case of no electric field, namely $H>P_{Z}^{2} / 2 M$ for regutar orbits. The numerical surface of section plots agree with the predicted criterion.

There are two major effects of the electric field on orbits. (1) All particles are confined in a spatial region of $b \leq \varepsilon_{d}^{-1}$ electrostatically. Particles with $H>P_{2}^{2} / 2 M$ still behave very regularly. (2) Since the energy of a particle should be greater than or equal to the minimum value of the potential energy $H_{*}$,

$H \geq H_{*}$

where

$$
H_{\star}=\left\{\begin{array}{l}
0 \text { for } \underline{E}_{0}=0 \\
\frac{T}{e} \ln \left(1-\alpha E_{d} P_{z}\right) \text { for } \underline{E}_{0} \neq 0
\end{array} .\right.
$$

Therefore the presence of $E_{0}$ eliminates the particles of energy values between 0 and $H_{\star}$. Since this is the regime where most arbits behave stochastically, it is equivalent to eliminating some of the stochastic orbits.

To illustrate the population of each rjige of motion in the two different equilibria more clearly, the allowable region of each equilibrium in $\left(H, P_{Z}\right)$ space is plotted in Fig. 6.1 along with the dividing curves of each type of motion. 
I 39

The allowable region in $\left(H, P_{2}\right)$ space of a confined plasma is determined by the following conditions: (1) The energy of a particle should be greater than or equal to the minimum potential energy in the system. (2) The energy of a particle should be less than the patentil energy at the plasma boundary to be confined. We consider only the absolutely confined orbits, in other words no "bounce" particles that are reflected at the plasma boundary are included.

If we apply the two conditions to the equilibrium with no electric field (studied in Chap. 111), the allowable region is

$$
\frac{P_{z}^{2}}{2 M} \leq H \leq \frac{1}{2 M}\left(P_{z}-P_{z B}\right)^{2} \text { for } P_{z} \leq 0
$$

and

$$
0 \leq H \leq \frac{1}{2 M}\left(P_{z}-P_{z B}\right)^{2} \text { for } P_{z} \geq 0 \text {, }
$$

where

$$
P_{z B}=\frac{e a_{a}}{2 c a} b_{B}
$$

with $b_{B}$ being the value of at the plasma boundary. The region is shown in Fig. 6.1(a) with boundary curves of (a), (b) and (e) in the figure.

For our VF equilibrium, all particles are confined energetically. Therefore only the first constraint remains. The allowable region is

$$
\frac{P^{2}}{2 M} \leq H \text { for } \quad P_{z} \leq 0
$$


and

$$
\frac{1}{2 M}\left(P_{z}-\alpha \phi_{m n}\right)^{2}-T \ln \left(1-\varepsilon_{d} \phi_{m n}\right) \leq H \quad \text { for } P_{z} \geq 0 \text {, }
$$

where $\phi_{m n}\left(0<\phi_{m n}<c_{d}^{-1}\right)$ is the value of s at which the minimum of the effective potential of Eq. (6.13) occurs. The boundary curves are shown as curves (a) and (c) in Fig. 6.1(a). The dividing curve of $\mathrm{J}_{y^{-}}$ conserving orbits to stochastic orbits, $H=P_{z}^{2} / 2 M$, is shown as curve (d) of Fig. 6.1, which is valid for all $P_{z}$ values for the equilibria with $\underline{E}_{0}=0$ and valic for the VF equilibria as long as $\varepsilon_{d} P_{z} / a<<1$. The dividing curve of stochastic orbit to $\mu$-conserving orbits, $H=$ $4 \times 10^{-5} \mathrm{P}_{z}^{2} / 2 \mathrm{M}$, is essentially $\mathrm{H}=0$ in the allowable region of both equilibria. Comparing the two allowable regions it is clear that the VF equilibrium has more regular orbits than the other.

If we take FRX-B or C parameters at LANL, more orbits are stochastic if the size of the plasma increases and more orbits are regular if the temperature of the ions increases for both equilibria. Here regular means $J_{y}$-conserving only.

\section{The Trial Function}

Our goal is to explain the long lifetime of a plasma in FRC in spite of the fact that the MHO theories predict fast growing modes. Tinerefore, our interest is in the most unstable MHD modes in the system. In particular, we will investigate the axial shifting $(m=1)$ displacements focussing on the modes corresponding to lower toroidal mode numbers (low $n$ ). 
To reduce the computational work, we will find an analytic form of a displacement vector close to the shifting modes from the known properties of the modes instead of solving the eigenvectors of idear MHD equations exactly computationaliy.

If we summarize the properties of those modes described in Chap. $V$, they are (1) incompressible $(\underline{\nabla} \xi=0)$. (2) For elliptical flux surfaces $\underline{\xi}$ is rigid axial $\left(\xi_{y}=0\right.$ and $\underline{\xi}(x, y, z, t)=\underline{\xi}(b, z, t)$ where $\left.b \equiv \varepsilon^{2} x^{2}+y^{2}\right)$, and (3) primarily internal i.e. the axial displacement $\xi_{x}$ vanishes at the separatrix. From the fact that the displacemnt vector $\underline{\xi}$ depends on $x$ and $y$ only through $b$ the surface term of MHD $s W$ is guaranteed to vanish. From $\underline{\nabla} \cdot \underline{\xi}=0$ and $\xi_{y}=0, \xi_{z}$ is determined from $\xi_{x}$ which leads to

$$
\xi(\phi, z, t)=\left[\xi_{x}(\phi) \hat{x}-\frac{2 \varepsilon^{2} x}{k} \frac{d \xi_{x}(\phi)}{d \phi} \hat{z}\right] e^{i(k z-\omega t)}
$$

for $k \neq 0$. Notice that $\xi_{x}$ is the only function to be chosen and must be a differentiable function of so that $\xi_{z}$ does not diverge.

In determining the structure of $\xi_{x}(\phi)$ the oscillation theorem is useful. The theorem shows that the most unstable MHD mode in a circular Z-pinch is the lowest radial mode. Hence it is suggestive to look for only the lowest radial mode structures. In particular, we will be interested in two different radial structures, one with a maximum displacement at the null (type I) and the other with zero displacement at the nuIl (type II). In both cases, the displacement vanishes at the separatrix. Simple analytic forms of the radial structures of $\xi_{x}(b)$ are 


$$
\xi_{x}(b)=\left(b-\phi_{s}\right)^{2} \quad \text {; for type } 1 \text {, }
$$

and

$$
\xi_{x}(b)=b\left(b-\phi_{s}\right)^{2} \quad ; \text { for type II, }
$$

where $b_{S}$ is the value of $b$ at the separatrix as shown in Fig. 6.2. The full expression of the trial functions is then for type I

$$
\underline{\xi}(\phi, z, t)=\left[\phi\left(\phi-\phi_{s}\right)^{2} \hat{x}-\frac{4 \varepsilon^{2} x}{k}\left(\phi-\phi_{s}\right) \hat{z}\right] e^{i(k z-\omega t)}
$$

and for type II

$$
\underline{\xi}(\phi, z, t)=\left[\phi\left(\phi-\phi_{s}\right)^{2} \hat{x}-\frac{2 \varepsilon^{2} x}{k}\left(\phi-\phi_{s}\right)\left(3 \phi-\phi_{s}\right) \hat{z}\right] e^{i(k z-\omega t)}
$$

Traveling Waves vs. Standing Waves

The first question might be "Do they make any difference to the dispersion relation?" If yes, then, "How much ?" The answer is, "Yes, very much in genera?."

As far as ideal MHD theory goes, eigenvectors can be expressed in either a standing wave form or a traveling wave form and the eigenvalues do not depend on the form of the eigenvectors. However when we take into account the gyro orbits, the eigenvalues are of course dependent on the choice of the form of eigenvectors. 
The domain of the VF dispersion function space is the right half plane of the complex dispersion functional space. The domain corresponding to the standing waves are the positive real axis which is of measure zero to that corresponding to traveling waves. Also the property of the expectation values of the standing waves is singular in the sense that we discussed in Chapter IV.

For these reasons we have chosen the trial functions in the form of a traveling wave in $z$.

E. Evaluation of Growth Rates (I)

Because of the complexity of the problem, we are not solving a dispersion matrix. Instead we will simply estimate the growth rates $(Y)$ by evaluating the expectation value of $\omega$ of the VF dispersion functional by employing the trial functions described in the previous section. The error in $y$ obtained by the chosen trial functions to the exact values has not been investigated yet, but is hoped to be small. We evaluate the three terms of the VF dispersion functional one by one: the first two terms can be evaluated analytically due to our analytic form of the trial functions and the third term is evaluated numerically.

\section{E.1 Evaluation of $s W$}

In general sw has three parts; vacuum, surface and fluid terms. The surface and the yacuum terms do not contribute to our calculation 
of ow since we restrict ourselves to internal modes. The $8 \mathrm{~W}$ in the VF dispersion functional is the ideal MHD energy variational for incompressible displacements. Denoting the fluid part of $\delta \mathrm{W}$ as $\delta \mathrm{W}_{F}$ we obtain a simple expression for $8 \mathrm{~W}$ :

$$
\begin{aligned}
\delta W & =\delta W_{F}(\underline{\nabla} \cdot \underline{\xi}=0) \\
& \left.=\frac{1}{2} \int \underline{d \underline{x}} \int \xi^{\star} \xi: \nabla \nabla\left(P_{0}+\frac{B_{0}^{2}}{8 \pi}\right)+\frac{1}{4 \pi}\left|\underline{B}_{0} \cdot \nabla \underline{\xi}\right|\right\}^{2},
\end{aligned}
$$

where $P_{0}(\underline{x})=n_{0}(\underline{x}) T$.

For trial function I of Eq. (6.19a) we obtain

$$
\begin{aligned}
& \underline{\xi}_{\underline{k}}^{\dot{\xi}}: \underline{\nabla \nabla}\left(P_{0}+\frac{B_{0}{ }^{2}}{8 \pi}\right)=-\xi_{x}{ }^{2}(\phi) \frac{\varepsilon^{2}}{\pi}\left(\frac{B_{a}}{2 a}\right)^{2} e^{2 \gamma t} \\
& \underline{B}_{0} \cdot \underline{\nabla \xi}=-\frac{4 y \varepsilon^{2}}{i k} \frac{B_{a}}{2 a} \xi_{x}{ }^{\prime}(\phi) e^{i(k z-\omega t)}
\end{aligned}
$$

and finally

$$
\delta W=\frac{\varepsilon^{2}}{2 \pi}\left(\frac{B a}{2 a}\right)^{2} \int \frac{d \phi d \theta d z}{2 \varepsilon}\left[-\xi_{x}^{2}+\frac{2 \varepsilon^{2}}{k^{2}}\left(\xi_{x}{ }^{\prime}\right)^{2}\right],
$$

where the variables and $\theta$ are transformed from Cartesian coordinates as $x=\frac{\sqrt{\phi}}{\varepsilon} \cos \theta$ and $y=\sqrt{6} \sin \theta$ and so $d \underline{x}=\frac{1}{2 \varepsilon} d \phi d \theta d z$. In evaluating Eq. (6.21) we average $\sin ^{2} \theta$ and $\cos ^{2} \theta$ each to $\frac{1}{2}$.

The first term of Eq. (6.21) is the pressure driven destabilizing term while the second term is the magnetic compression energy associated with the field line compression in $z$, thus makes a stabilizing 
effect for all $\mathrm{k}$ values. From the incompressibility condition, $\xi_{2}$ is small when $k$ is large and vice versa. Therefore, the compression becomes less severe as $k$ increases, and vanishes in the limit of $k \rightarrow \infty$, which means that the most dangerous situation is for large $k$. If $k$ is small enough, the compression energy may tike over the destabilizing effect, showing stability against the chosen displacerient.

It is worth evaluating the transition values of $k, k_{c}$, for each of our trial functions so that we can work on only unstable $k$ values. The values of $\delta \mathrm{W}$ and $\mathrm{k}_{c}$ for the trial function I are:

$$
\delta W=-\frac{\varepsilon \pi}{2 k}\left(\frac{B a}{2 a}\right)^{2} \frac{\sigma_{s}^{3}}{5}\left(1-\frac{10 \varepsilon^{2}}{3 k^{2} b_{s}}\right)
$$

and

$$
k_{c}=\varepsilon \sqrt{\frac{10}{3 \phi_{s}}}=.09 \text { for } \varepsilon=0.1 \text { and } \phi_{s}=4
$$

which are the parameters of FRX-B at Los Alamos National Laboratory. Likewise, for type II the threshold value of $k_{c}$ is $=.13$ for the same values of $\varepsilon$ and $\phi_{S}$, $\delta W>0$ for the displacements with $k<k_{c}$. This does not necessarily mean that the system is MHD stable but simply means that the chosen displacements with $k<k_{c}$ are stable.

The relation between the wave vector $k$ of a cylindrical geometry and the toroidal mode number $\mathbf{n}$ of a toroidal system is obtained as $k=n / R$ where $R$ is the distance between the null and the axij by equating the wavelength $\lambda$ of the two configurations: $\lambda=2 \pi / k$ in a cylindrical system and $\lambda=2 \pi R / n$ in a toroidal system. If we take $R=2 \mathrm{~cm}$ of a FRC-B, $k=n / 2$ where $n$ is a nonzero integer. So $|k|>k_{C}$ 
for all nonzero integer $n$. This indicates that the MHD shifting unstable modes of a FRC-B are also unstable in a cylindrical Z-pinch, which gives us more confidence in the choice of our trial functions and also in making a cylindrical approximation of a compact torus in the absence of the toridal field. The above argument applies also to the case of FRC-C where $R=5 \mathrm{~cm}$. If we take standing wave trial functions, one obtains exactly the same ow as in Eq. (6.22) except a factor $1 / 2$ which comes from the normalization factor. Thus, as far as MHD oW goes, there is no difference whether one chooses traveling waves or standing waves as trial functions. The argument given above applies directly to the case of standing wave trial functions wichout any modifications.

\section{E.2. Evaluation of the Cross Term (I $)$}

Above all, it is not hard to see that $I_{c} \equiv i \frac{e}{c} \int d \underline{x} n_{0} \underline{\xi}^{*} \cdot \underline{\xi} \times \underline{B}_{0}$ has the property that $I_{c}=I_{c}{ }^{\star}$. Therefore if the spatial dependence of the displacement vector is real, $I_{c}$ vanishes identically. Otherwise $I_{c}$ is real. Since we have assumed the time dependence of the displacement as $e^{-i \omega t}$, the spatial part of the displacement is real for a standing wave form and complex for a traveling wave form. If we express our trial functions in traveling wave form in $z$

$$
\underline{\xi}^{*} \cdot \underline{\xi} \quad \times \underline{B}_{0}=\frac{8 \varepsilon x^{4} x^{2}}{i k}\left(\frac{B}{2 a}\right) \xi_{x} \frac{d \xi x}{d \phi}
$$

Transforming $d \underline{x}$ to $\frac{1}{2 \varepsilon} d \dot{d} d \theta d z$, as before, and integrating by parts we obtain 


$$
I_{c}=-2 \varepsilon^{2} n_{0}(0)\left(\frac{e}{c}\right)\left(\frac{a}{2 a}\right)\left(\frac{1}{k}\right) \int \frac{d \phi d e d z}{2 \varepsilon}\left(1-2 \varepsilon_{d} \phi\right) \xi_{x}{ }^{2}(\phi) \quad .
$$

Since the integrand $1-2 \varepsilon_{d} 6$ changes sign at $\phi=1 / 2 \varepsilon_{d}$ which is within the pseudo separatrix; the 2 nd term of the VF dispersion functional is either positive or negative depending on the structure of $\xi_{x}(b)$. Roughly speaking, for $k>0$, the 2 nd term is positive for displacement localized in the outer region ( $\phi>1 / 2 \varepsilon_{d}$ ) of the plasma, and is negative for displacement localized near the nult. For $k<0$, the situation is just the opposite to that of $k>0$.

\section{E.3 Evaluation of the Orbit Integral Term (I}

The expression of the orbit integral terms $\left(I_{a b}\right)$ of the VF dispersion functional is transformed to Eq. (6.25) from Eq. (6.24) below, by introducing dynamical spectral densities. 5 First we summarize down the tranformation of the formalism by numbering each step for the elongated $Z$-pinch and then the numerical method corresponding to each step is discussed. For convenience, we write down the definition of the orbit term

$$
I_{o b} \equiv i_{\omega} e^{2} \int d \underline{z} \xi^{*} \cdot\left(\underline{E}_{0}+\frac{1}{c} \underline{v} \times B_{0}\right) \frac{d F}{d H} \int d t^{\prime} \underline{\xi} \cdot\left(\underline{E}_{0}+\frac{1}{c} \underline{v} \times \underline{B}_{0}\right) \text {. }
$$

For an elongated Z-pinch, $H$ and $P_{Z}$ are constants of motion (denoted by I in Chap. IV), therefore we transform $z$ to $\left(H, P_{z}, \Gamma\right)$ where

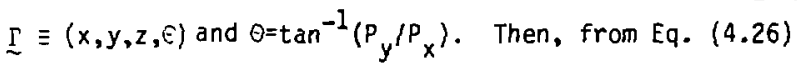


148

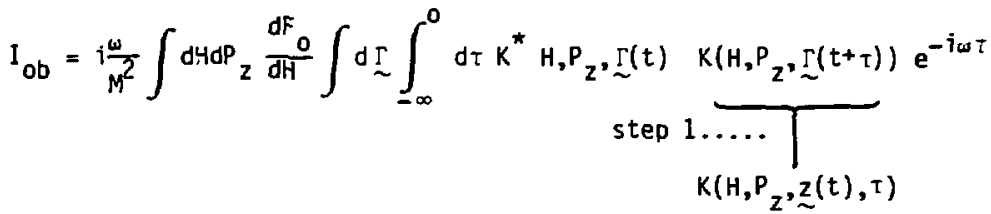

$$
\begin{aligned}
& =i \frac{\omega}{M^{2}} \int d H d P_{z} \frac{d F}{d H} \int d \tau \underbrace{\int d \Gamma \underset{\sim}{\sim} \mathscr{K}\left(H, P_{z}, \Gamma(t), \tau\right)}_{\text {step } 2 \ldots \ldots} \\
& Q\left(H, P_{2}\right)\langle\mathcal{S}\rangle\left(H, P_{2}, \tau\right) \\
& d_{\beta} \mathcal{P}\left(\beta ; H, P_{2}\right) e^{i \beta \tau} \\
& =\frac{\omega}{M^{2}} \int d B d H d P_{Z} \frac{d F_{O}}{d H} Q\left(H, P_{Z}\right) \frac{\Phi\left(B ; H, P_{Z}\right)}{B-\omega} \\
& =\frac{\omega}{M^{2}} \int d \beta \frac{1}{B-\omega} \int d H \frac{d F_{O}}{d H} \int d P_{z} Q\left(H, P_{z}\right) \mathcal{P}\left(B ; H, P_{z}\right) \\
& \text { step } 5 \text { step } 4 \\
& =\omega \int d B \frac{S\langle B\rangle}{B-\omega}
\end{aligned}
$$

where $S(B)=\frac{1}{M^{2}} \int d H \frac{d F_{D}}{d H} \int d P_{z} Q\left(H, P_{z}\right) \Phi\left(B ; H, P_{Z}\right) \quad$ and

$$
\mathfrak{K}(z(t)) \equiv K^{*}(\underset{\sim}{z}(t)) K(\underset{\sim}{z}(t+\tau)) .
$$

Initial Conditions (Step 0)

$H$ and $P_{2}$ values are given at regular grid points in the allowable region shown in Fig. 6.3. Since $f_{0}$ decays exponentially with respect to energy (notice that $\frac{\mathrm{dFo}}{\mathrm{dH}} \sim F_{0}$ ) and also there are some algebraic 
dependences of energy in the integrand of the $\Gamma$-phase integral the maximum value of $H$ is chosen $-5 T$. The rest of the initial conditions in $(\theta, \theta, z, \theta)$ space are chosen randomly. For given values of $H$ and $P_{z}$, a definite range of $b$ is determined and $b$ is randomly chosen in the allowable range. The region $\phi(x, y)$ are plo:ted qualitatively in Figure 6.3. $\theta, \theta$ and $z$ are also chosen rardomly: ihe range of $\theta$ and $\Theta$ are $[0,2 \pi]$ and the range of $z$ is $[0, \lambda=2 \pi / k]$ where $k$ is the wave vector in $z$ of the trial function.

The relation between the variables $\left(H, P_{z}, \theta, \theta, z, \phi\right)$ and the cartesian variables $\left(x, y, z P_{x}, P_{y}, P_{z}\right)$ are as follows:

$$
x=\frac{\sqrt{\phi}}{\varepsilon} \cos \theta, y=\sqrt{D} \sin \theta, P_{x}=P \cos \theta \text { and } P_{y}=P \sin \theta
$$

where

$$
P^{2}=P_{x}^{2}+P_{y}^{2}=\frac{2}{M}\left(H-T \ln \left(1-\varepsilon_{d} \phi\right)\right)-\left(P_{z}-\alpha \phi\right)^{2}
$$

$z$ and $P_{z}$ are the same in both coordinates.

Step 1

Once the inital conditions $z(t)$ are assigned, we make a record of $K(z(t+\tau))$ as a function of $\tau$ by numerically integrating the unperturbed equations of motion.

Step 2

Our main problem in dealing with orbit effects is how to evaluate $\int d \underset{\sim}{\mathcal{K}(z}(\underline{z}(t), \tau)$ efficiently including all kinds of orbit behavior where we have used the definition of $k$ in Eq. (6.25). The integral over $I$ can be obtained in principle by choosing a large enough number of 
particles to represent the four-dimensional Ispace reasonably well. To obtain the correlation as a function of $\tau$ one should follow the particles at least for a time $\tau$. This would take incredibly long computer time. Here we will introduce a very efficient way of evaluating the $\Gamma_{\sim}$ space averaged correlation function $\langle\mathcal{K}\rangle_{I}\left(H, P_{2}, \tau\right)$ regardless of the behavior of orbits.

First from the fact that $\int d \Gamma \mathcal{\sim}(z(t), \tau)$ is independent of $t$, we write

$$
\begin{aligned}
& \int d \Gamma \underset{\sim}{L}\left(z\left(t_{0}\right), \tau\right)=\frac{1}{T} \int_{0}^{T} d t \int d \Gamma \mathcal{\sim} \mathcal{K}\left(z_{0}\left(t_{0}\right), \tau\right) \\
& a \Gamma\left(t_{0}\right) \quad \partial \quad \partial\left(t_{0}\right)
\end{aligned}
$$

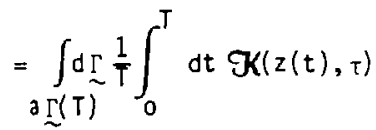

$$
\begin{aligned}
& =\int_{a \underset{\sim}{\Gamma(\tau)}} d \underset{\tau}{\Gamma}\langle\mathcal{K}(z(t), \tau)\rangle_{t}
\end{aligned}
$$

If the dynamical system possesses mixing properties, one initial condition is good enough to evaluate Eq. (6.27) and

$$
\begin{aligned}
& \left.\int d \underset{\sim}{\mathcal{X} Z} z(t), \tau\right)=\mathcal{Q}\left(H, P_{z}\right)\langle\mathcal{K}(z(t), \tau)\rangle_{t} \\
& \text { a } \Gamma(t)
\end{aligned}
$$

If the orbits are regular, then each orbit stays on an invariant torus. Therefore we need many initial conditions to describe the phase integral reasonably well. Let $z_{j}(t)$ be the initial conditions then the subscript $i$ not only labels the particle but also lat. Is the invariant 
torus. Suppose we have $N$ initial conditions, then the last expression of Eq. (6.27) can be approximated in most cases as

$$
\sum_{i=1}^{N} W_{i}\left(H, P_{z}\right)\left\langle\mathcal{K}\left(z_{i}(t), \tau\right)\right\rangle_{t}
$$

where $\mathcal{V}_{j}\left(H, P_{z}\right)$ is the volume between the invariant tori represented by the $i^{\text {th }}$ particle or $i^{\text {th }}$ invariant torus. In the continuous limit $N \rightarrow \infty, \mathcal{O}_{i}\left(H, P_{2}\right)$ is equivalent to $Q\left(H_{1} P_{2}, I_{3}\right)$ where $I_{3}$ denotes the third invariant. If the orbit behaves ergodically (more precisely, mixing) in the subdomain denoted by $W_{j},(6.29)$ equals $(6.27)$ if we adopt the ergodic assumption that the phase average is the sane as the time average in the limit $T \rightarrow \infty$. When the orbits are regular, the quantity $\mathfrak{K}$ averaged over the invariant torus is assumed to represent the average in a volume around the torus. This assumption is not bad as long as the quantity of interest does not change dramatically with respect to the third invariant.

To explain the properties visually, in Fig. 6.4 we silow a phase space, more precisely a $\underset{\sim}{\Gamma}$ space. The figure can be used as the total phase space $(H, P, \Gamma)$ with $N$ initial conditions and its evolution in time. In particular we show two volume elements $v_{1}\left(t_{0}\right)$ and $v_{2}\left(t_{0}\right)$ where $\mathcal{V}_{1}\left(t_{0}\right)$ is represented by an ergodic orbit and $\mathcal{V}_{2}\left(t_{0}\right)$ by a regular orbit. For the ergodic case, the relative position of the boundary of $\boldsymbol{V}_{1}(t)$ and the position $z_{1}$ changes in time thus covering the volume $Q_{1}(t)$ everywhere, On the other hand for a regular orbit the relative position is fixed. Regardless of the orbit behavior, in order that the 
expression (6.29) be a valid approximation of (6.27) the time averaged quantity $\langle\mathcal{J}\rangle_{t}$ should be ayeraged over a sufficiently long time so that the time averaged quantity is independent of the amount of time we averaged over. Now we assume that the initial conditions are distributed such that each initial condition represents the phase volume element of $\mathcal{Q}\left(H, P_{z}\right) / N$. Then, $Q_{i}\left(H, P_{z}\right)$ of expression (6.29), which is from the $i^{\text {th }}$ volume element described by the $i^{\text {th }}$ initial condition $z_{j}\left(t_{0}\right)$ at $t=t_{0}$ is also $Q\left(H, P_{z}\right) / N$ since each volume element is conserved by Hamiltonian flow. Thus

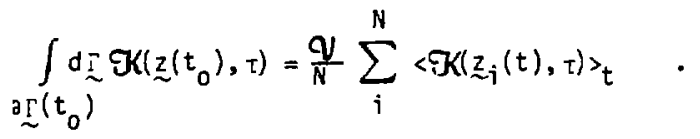

As is mentionerj earlier, the time integral should be performed over a sufficiently long time that the time average is independent of T. The cutoff time is normally the correlation time beyond which the contribution is exponentially small. If the orbits are ergodic the correlation time is short but the regular orbits usually have long correlation times (the correlation tends to decay algebraically rather than exponentially). Sometimes the correlation never decays (e.g. in a 1-0 simple harmonic oscillator).

However, with the help of the convolution property, ${ }^{5}$ the time integral of the correlation is replaced by the frequency integral of a function of the spectrum of the $K(z(t))$. Introducing a time fourier transformation 


$$
K(z(t))=\sum_{i} \Phi\left(\beta_{i}\right) e^{i^{\beta} t} \Delta \beta_{i}
$$

we transform the time integral as follows:

$$
\begin{aligned}
& \frac{1}{T} \int K^{*} K d t=\frac{1}{T} \int_{0}^{T} d t \sum \Phi^{*}\left(\beta_{j}\right) e^{-i \beta_{j} t} \Delta \beta_{i} \sum \Phi\left(\beta_{j}\right) e^{i \beta_{j}^{\prime}(t+\tau)} \Delta \beta_{j}^{\prime} \\
& =\frac{1}{T} \int_{0}^{T} d t \sum_{i, j} \Phi^{*}\left(\beta_{j}\right) \Phi\left(B_{j}\right) e^{-i\left(\beta_{j}-\beta_{j}^{\prime}\right) t} e^{i \beta_{j}^{\prime} \tau} \Delta B \Delta B^{\prime} \\
& =\sum_{i}\left|\mathcal{P}\left(\beta_{i}\right)\right|^{2} e^{i \beta_{i} \tau}(\Delta \beta)^{2}
\end{aligned}
$$

Notice that the last equality holds only when $k\{z(t)\}$ is periodic with period $T$, which will be assimed in the numerical analysis. The correlation is now replaced by the spectrum sum of $k(z(t))$. If we follow the orbit long enough to pick up all the interesting frequencies such as gyrofrequencies and bounce frequency the correlation is well approximated. Consequently the correlation has all the interesting frequencies which gives correct dynamical spectral densities.

In any event, we have finite data say from time 0 to $T$. Then to obtain the spectrum of the data, periodicity of the data is assumed. In general the amplitude at $t=T$ is not the same as that of $t=0$. This will bring in non-physical high frequency spectrum components, i.e. so-called "noise". A data window is introduced to get rid of tre noise. A smooth symmetrical function with respect to $t=T / 2$ and zero 
amplitude at $t=0$ and $T$ is multiplied to $K(\underset{\sim}{z}(t))$. The high frequency noise is then reduced but because of this artificial function, the correlation function always decays (including the case of a 1-0 simple harmonic oscillator). But the spectrum of the correlation is not affected too much ( $r \in f e r$ Fig. 7.2). Therefore time average of each orbit, $\langle\mathcal{G}\rangle_{t}$, is evaluated by the following steps

(1) Apply data window to $K(z(t))$

(2) Make a Fourier transformation of the data-windowed record $K(z(t))$ to make $\boldsymbol{P}(s)$

(3) Evaluate $\Phi(B)^{2}$

(4) Do an inverse Fourier Transformation Thus,

$$
d \underset{\sim}{\Gamma} \mathcal{W}=\frac{O\left(H, P_{2}\right)}{N} \sum_{i=1}^{N} \Phi\left(\beta_{i}\right)^{2} e^{i \beta_{i} T}\left(\Delta \beta_{i}\right)^{2}
$$

\section{Step 3}

Make a Fourier Transformation of the correlation to get power spectral'densities of $\Phi\left(\beta ; H, P_{Z}\right)$.

Step 4

The $P_{Z}$-integral is easily evaluated by suming the spectral densities of $\Phi\left(B ; H, P_{Z}\right)$ over all grid points of $P_{Z}$ values of the same $H$.

$$
\Phi(B, H)=\sum_{i=1}^{N_{P}} Q\left(H, P_{Z i}\right) \Phi\left(B ; H, P_{Z i}\right) \Delta P_{Z}
$$


where $\Delta P_{Z}$ is the interval of the grid points in $P_{Z^{*}}$. The range of $P_{z},\left[P_{z m n}, P_{z m x}\right]$, is determined as a function of $H$.

$$
\begin{aligned}
& P_{z \pi n}=-\sqrt{2 H} \\
& P_{z m x}=1-\varepsilon_{d}^{-1} \exp (-\mathrm{eH} / T) .
\end{aligned}
$$

The accessible phase volume in $I$ space by particles of $H$ and $P_{Z}$ is

$$
\boldsymbol{W}\left(H, P_{z_{i}}\right)=\int \underline{d x d} \theta=\int \frac{d \Theta d \theta d z d \phi}{2 E}=\frac{(2 \pi)^{3}}{2 \varepsilon k}\left(\phi_{m x}\left(H, P z_{i}\right)-\phi_{m n}(H, P z i)\right)
$$

where $\phi_{\mathrm{mx}}$ and $\phi_{\mathrm{mn}}$ are the upper and lower bounds of for a given $H$ and $P_{z i}$.

Step 5

Adding the spectral densities $(B ; H)$ over all different grid values of $H_{i}$ with a proper weighting factor we obtain the total spectral density

$$
S(B)-\sum_{i=1}^{N_{H}} \Phi\left(B ; H_{i}\right) \Delta H_{j},
$$

where

$$
\Delta H_{i}=\sum_{H_{i-1}}^{H_{i}} e^{-H / T} d H=T\left|e^{\frac{-H_{i-1}}{T}}-e^{-T}\right| \text {. }
$$


Due to the exponentia] cutoff and the algebraic dependence in energy, the range of $H$ is truncated at $\sim 5 T$.

Now we have obtained $S(B)$ at discrete values of $B, B_{i}=(\Delta B) \cdot i$ where $i$ is integer of $\left[-N_{B}, N_{B}\right]$, which gives the orbit integral term of the VF dispersion functional in the following form:

$$
I_{o b}=\omega \sum_{i=-N_{B}}^{N_{B}} \frac{S\left(B_{i}\right)}{B_{i}^{-\omega}} .
$$

E.4 Finding the Roots of a Dispersion Re?ation

Eventually our dispersion relation looks as follows

$$
2 \delta w+\omega I_{C}+\omega \sum_{i=-N_{B}}^{N_{B}} \frac{S\left(B_{j}\right)}{\beta_{i}^{-\omega}}=0,
$$

where $\delta \mathrm{W}$ and $\mathrm{I}_{C}$ are some numbers.

There arise two difficulties in finding the roots of Eq. $(6.39)$. The first is that it has $2 N_{B}+1$ poles. Whenever $\gamma$ is small and $\sigma=B_{j}$, the orbit term diverges. This problem can be eliminated by evaluating the orbit integral as follows for small values of $Y$.

$$
\int_{-\infty}^{\infty} \frac{S(\beta)}{\beta-\omega} d \beta=\int_{-\infty}^{\infty} \frac{S(\beta)}{\beta-\sigma} d \beta+i \pi S(\sigma)
$$


where the principal part can be transformed as

$$
\begin{aligned}
\int_{-\infty}^{\infty} \frac{S(\beta)}{\beta-\sigma} d \beta & =\int_{-\infty}^{\infty} \frac{S(\beta+\sigma)}{\beta} d \beta=\int_{-\infty}^{\infty} \frac{S(-\beta+\sigma)}{\beta} d \beta \\
& =\frac{1}{2} \int_{-\infty}^{\infty} \frac{S(\beta+\sigma)-S(-\beta+\sigma)}{\beta} d \beta
\end{aligned}
$$

Then, when the denominator vanishes the numerato also vanishes and when the denominator is smal? the numerator is small for a smooth function of $S(B)$.

Now we have

$$
2 \delta W+\omega l_{c}+\frac{\omega}{2} \sum_{i} \frac{S\left(B_{i}+\sigma\right)-S\left(-B_{j}+\sigma\right)}{B_{i}} \Delta B+\omega i \pi S(\sigma)=0
$$

The second difficulty is that the exact dispersion relation (6.39) is essentially a $2 \mathrm{~N}_{B}+2$ order polynomial and yields $2 \mathrm{~N}_{B}+2$ roots most of which are not physical. In order to obtain the right roots, the Nyquist Diagram method described in Chap. IV can be used to check whether the solution is physical.

A very simple approximated VF dispersion functional can be obtained from the fact that the VF model has small values of 0 when $\gamma$ is small. Therefore it is suggestive to do the Taylcr expansion of Eq. (6.41) around $\sigma=0$. Keeping only up to the second arder in $\omega$, we obtain 


\section{8}

$$
\begin{aligned}
25 W+\omega\left(I_{c}+\frac{1}{2} \sum_{i} \frac{S\left(B_{i}\right)-S\left(-B_{i}\right)}{B_{i}}+i \pi S(0)\right) \\
+\omega^{2}\left[\frac{1}{2} \sum_{i} \frac{S^{\prime}\left(B_{i}\right)-S^{\prime}\left(-B_{i}\right)}{B_{i}}\right]=0
\end{aligned}
$$

where the prime denotes the derivative with respect to $B$.

F. Evaluation of Growth Rates (II)

For the reasons described in Sec. D.2 of Chaf. IV, we discuss here the evaluation of the growth rates by using the second form of the dispersion functional of Eq. (4.31 a).

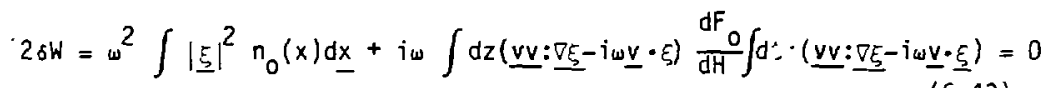

The first two terms are trivially evaluated.

The MHD growth rates are

$$
{ }_{\text {MHD }}^{2}=\frac{2 \delta \omega}{\int|\underline{\xi}|^{2} n_{0}(\underline{x}) d \underline{x}}
$$

where $\delta W$ is explicitly shown in Sec. E.I in this chapter.

The new orbit term involves the correlation of $\underline{v v}: \underline{\nabla \xi}-i \omega \underline{v} \cdot \underline{\xi}$ which has essentially three different correlations; two of them are the autocorrelations of $\underline{v v}: \underline{\nabla \xi}$ and $\underline{v}-\underline{\xi}$ respectively and the third is the cross correlation of $(\underline{v v}: \underline{\nabla \xi}) *$ with $\underline{v} \cdot \underline{\xi}$ as shown in Eq. (4.3Ib). 
It seems that more computation is involved for this dispersion formula than before but this gives better insight and may not require too much accuracy to see the kinetic effects. Furthermore, since we are interested in the low frequencies, the major correlation to MHD is

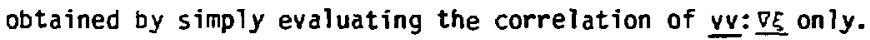

The rest of finding the growth rates is exactly the same as the previous case. 
160

REFERENCES AND FOOTNOTES (CHAP. VI)

1. J. P. Freidberg, Phys. Fluids 15, 1102 (1972).

2. W. T. Armstrong, R. K. Linford, J. Lipson, D. A. Plates and E. G. Sherwood, Phys. Fluids 24, 2068 (1981).

3. K. F. McKenna, W. T. Armstrong, R. R. Bartsch, R. E. Chrien, J. C. Cochrane, Jr., R. W. Newish, Jr., P. Klingner, R. K. Linford, D. J. Raj, E. G. Sherwood, R. E. Simon, and M. Tuszewski, Phys. Rev. Lett. 50, 1787 (1983).

4. J. S. Kim and J. R. Cary, Phys. Fluids 26, 2167 (1983).

5. R. B. Blackman and J. W. Tukey, Measurement of Power Spectra (Dover, New York, 1958). 


\section{FIGURE CAPTIONS}

Fig. 6.1 Character of orbits in FRX-B at LANL. Plot (a) shows the character of orbits in FRX-B in two different equilibria. For the VF equilibrium, the allowable region in $\left(H, P_{2}\right)$ space for a particle to be confined within the plasma boundary of FRX-B at LANL is the upper region of curves (a) and $(c)$. For the equilibria with no electric field, mentioned in the text, the region is above the 1 ines (a) and (e) and below the line (b). Roughly the region of $\mathrm{J}_{\mathrm{y}^{-}}$ conserving orbits and the region of stochastic orbits are divided by the curve (d) for both equilibria. The region of $\mu$-conserving orbits is negligible and not shown. The value $P_{Z B}$ is obtained by $P_{Z B}=\frac{a}{2 a c} \phi_{B}$ where $\phi_{B}$ is the value of $p \equiv \varepsilon^{2} x^{2}+y^{2}$ at the plasma boundary of FRX-B. Plot (b) shows the size dependence of the orbits in a VF equilibrium. The upper region of curves $(a)$ and $(b)$ is the al lowable region of $F R X-B$ and the upper region of curves (a) and (c) is the allowable region of $F R X-C$. Curve (d) divides the $\mathrm{J}_{\mathrm{y}}$-conserving orbits from the stochastic ones approximately. The $\mu$-conserving region is also not shown since it is negligible. $P_{Z B}$ is the same as in plot $(a)$. $P_{Z C}$ is obtained by replacing $\phi_{B}$ by $\phi_{C}$ where $\phi_{C}$ is the value of at the plasma boundary of FRX-C. Since $\phi$ is proportional to length squared and there is a coefficient of $1 / \mathrm{a}$, $P_{Z C}=2 P_{2 B}$ if $B_{a}$ is the same. 
Fig. 6.2 Two types of trial functions. Trial function I with a large displacement near the null is shown in plot (a). Trial function II which describes a displacement at some disiance from the null is shown in plot (b). Both are plotted with arbitrary amplitudes.

Fig. 6.3 A choice of $\left(H, P_{Z}\right)$ values and the allowable region in $x-y$ space. In the allowable region, the values of $H$ and $P_{Z}$ are chosen regulary shown as dots in the figure. The range of $x-y$ is well defined ance $H$ and $P_{z}$ are chosen. For a given $H$, the allowable region in the $x-y$ plane becomes large as $P_{z}$ increases from the minimum value of $P_{z}$ (Plot (a) to (c)). If $P_{z}$ is large enough to satisfy $P_{z}^{2}>2 m H$, particles cannot pass the axis. Then the $x-y$ range is confined to an annular ring as shown in plot (d). As the value $P_{2}$ increases further it approaches to a maximum value, the annular ring becomes narrower and narrower $(p \operatorname{lot}(e))$.

Fig. 6.4 Hamiltonian Flow. Three snapshots of the Hamiltonian flow of regular and stochastic orbits are shown. At each time, a particle represents the $\underset{\sim}{\Gamma}$ phase-space volume of $\frac{\mathcal{V}}{N^{-}}$if $\mathrm{N}$ particles are chosen, where $\mathcal{W}$ is the $\underset{\sim}{ }$ phase-space volume accessible by the particles. It shows how the phase average and the time average are related for regular and stochastic orbits. 

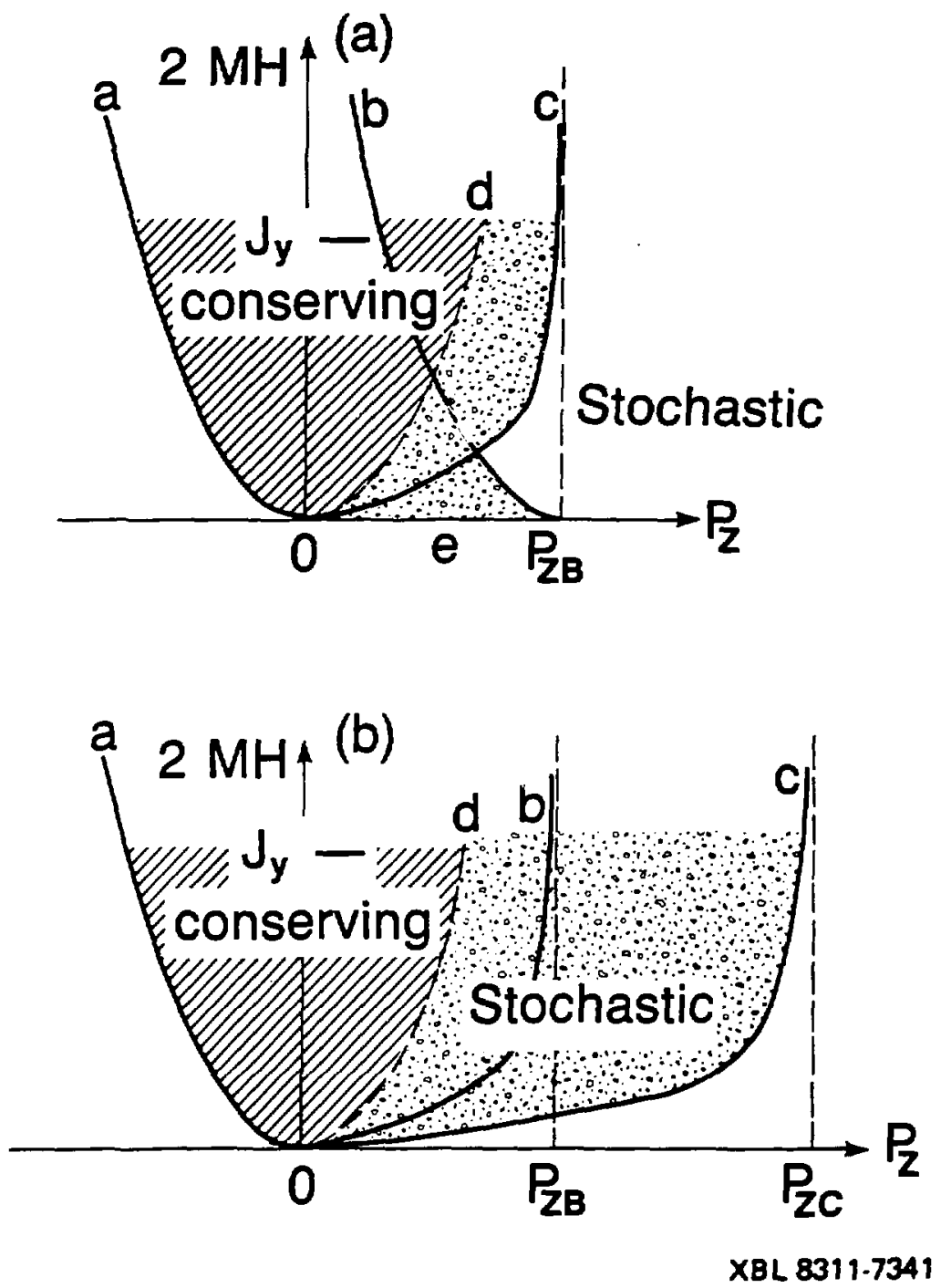

Figure 6.1 


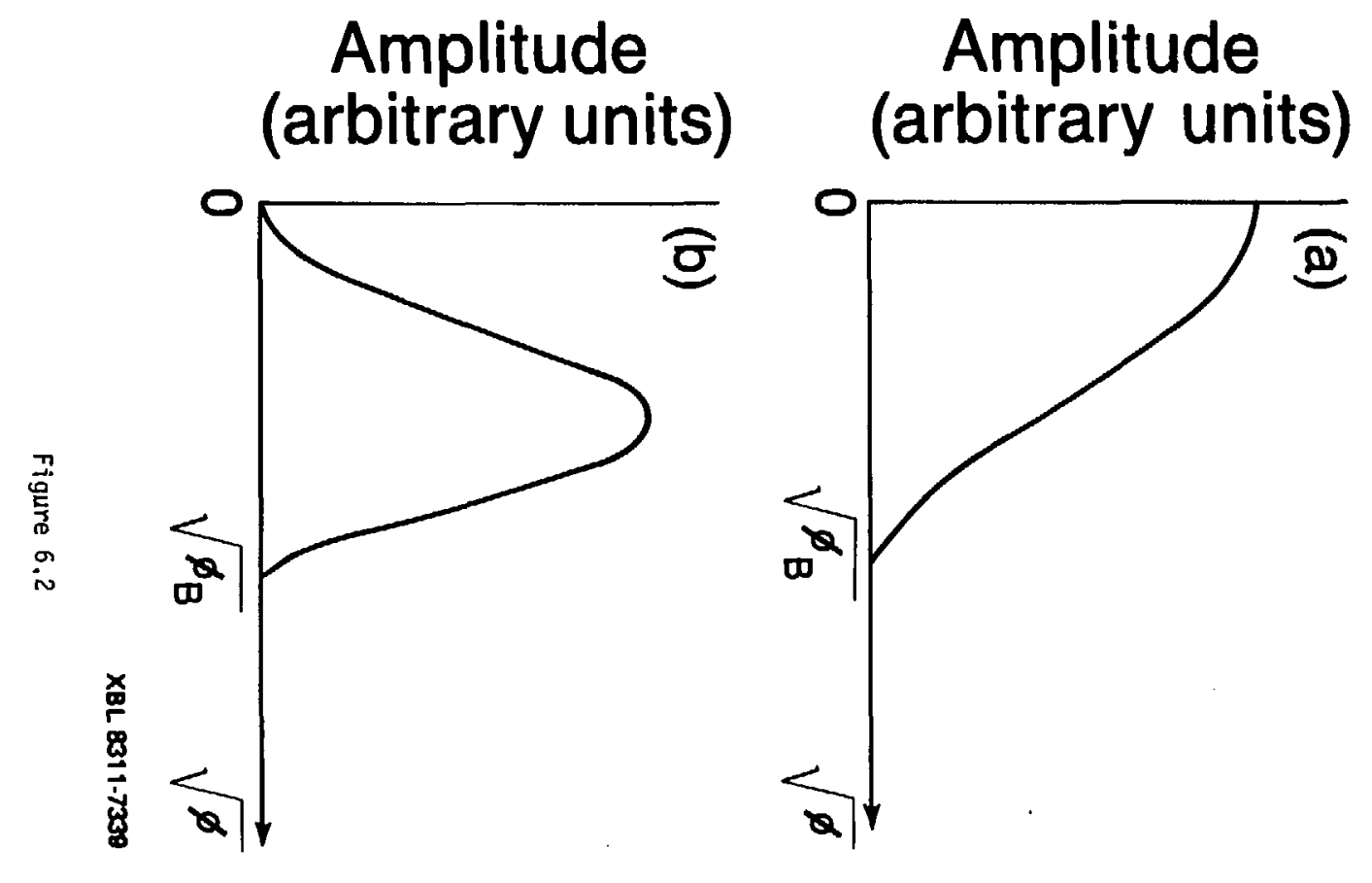


765
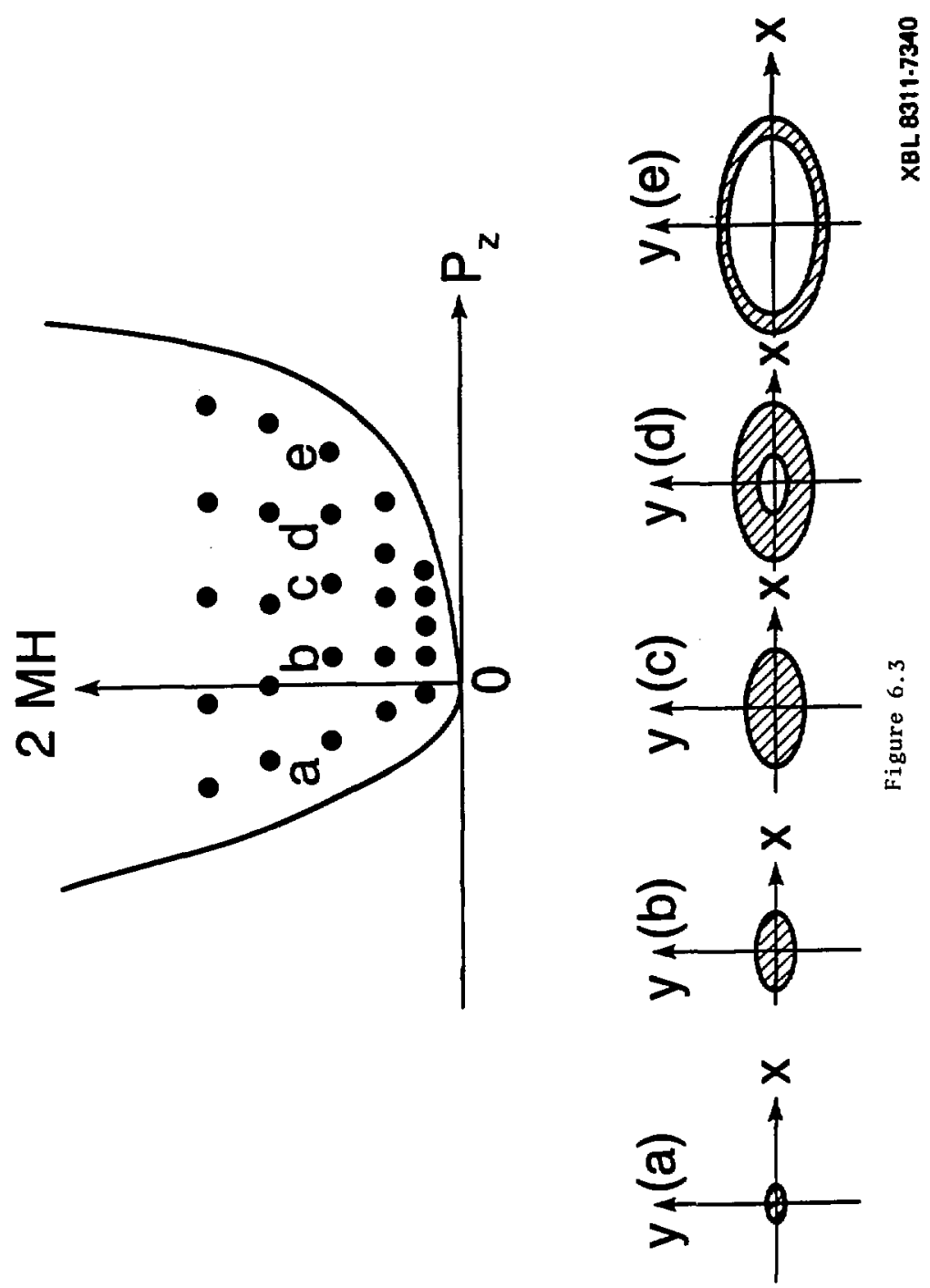


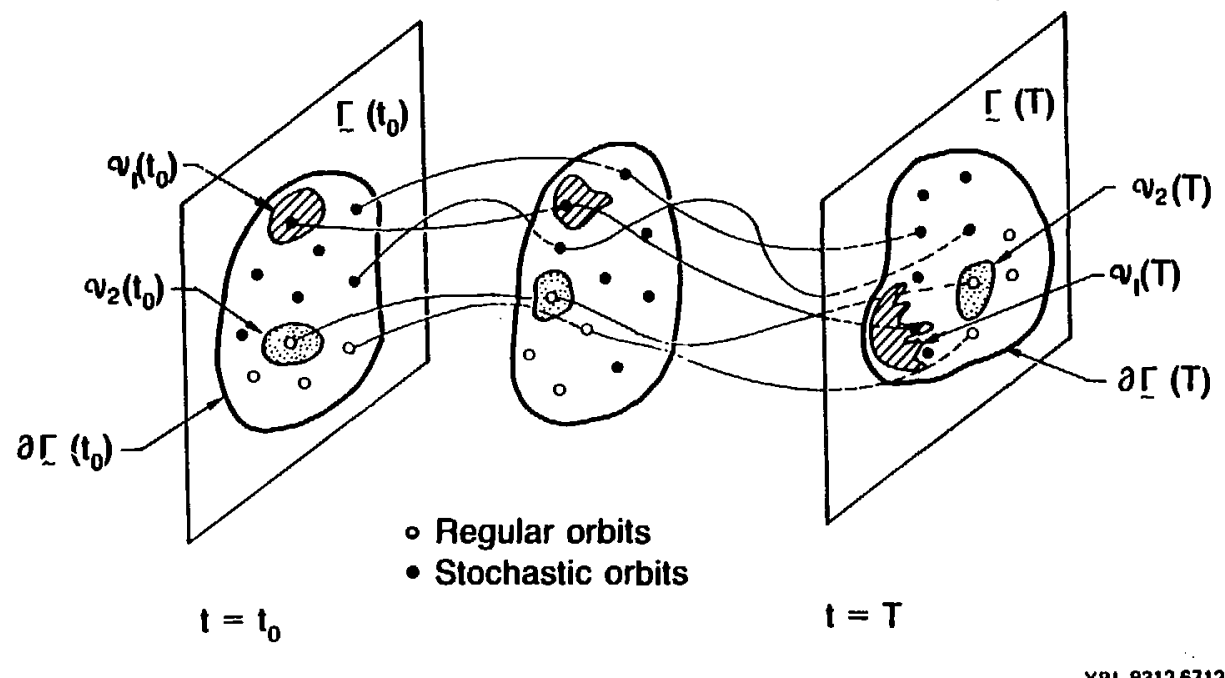

Figure 6.4 


\section{CHAPTER VII}

\section{RESULTS, DISCUSSIONS AND FUTURE WORK}

To el iminate all the unnecessary parameters, we do a scaling of the variables. This scaling reduces the stability problem so that it depends only on two parameters $\tilde{T}$ and $\tilde{k}$, where $\tilde{T} \equiv\left(r_{L} / a\right)^{2}$ parameterizes the temperature as a ratio of the gyro-radius to the size of the plasma and $\tilde{k}$ is equivalent to the toroidal mode nuliive " if the c!' inder were bent into a compact torus with major radius of $:$.

The main numerical work is to evaluate the dynamical spectral densities $S(\beta)$ which involves the phase average. The assumption that the phase average can be replaced by the time avarage if one choose many initial conditions botn for stochastic and regular orbits, is tested and found to be approximately valid. The saving in computation time, by replacing the phase average by the $t$ - te average, is enormous. It turns sut tha: only a few $(\leq 10)$ particles produce very typical spectral densities at a large scale for a given $H$ and $P_{z}$ in most cases.

Growth rates are evaluated firct by using the VF dispersion functional I (refer Eq. (4.29) or Eq. (7.23)) by following each orbit for about an MHD growth time. The results show almost the same growth rates as those of MHD. The reason is that the real part of $w$ is near zero where the accuracy of our spectral densities is in question. Therefore, before making definite remarks, one should follow each particle a lot longer. 
Instead of following the orbits for a longer time, we use the second formalism of the VF dispersion functional (refer Eq. (4.31) or Eq. (7.26)) since it gives kinetic corrections more directly, although the accuracy of the spectral densities near zero frequency still could be a problem. The kinetic corrections to MHD are studied by evaluating $S_{1}$ of Eq. (4.31b) only. For $\tilde{k}=10$ and 100 the growth rates are very much reduced for high $\tilde{T}$, and approach to that of MHD values as $\tilde{\mathrm{T}}$ decreases. We followed each orbit about 10 times longer to get a better consistent results for $\tilde{k}=10$. However, growth rates remain of the same order as those of MHD for $k=1$ even when the orbits were traced up to about 10 times longer than MHO growth time. The reason that we do not obtain reduced growth rates may be one or some of the following: (1) We still have not obtained the corrected spectrum near zero frequency. (2) The terms neglected in the second dispersion formalism are important. (This is very likely for low $\tilde{k}$ values). (3) It could be that ine toroidal effect, which is absent, of course, in our work, is important for the stability of low $\tilde{k}$.

The scalings of the variables are carefuliy described in Sec. A. The stability mechianism and a crude estimate of threshold values of $k$ are evaluated in Sec. B. Internediate steps of finding spectral densities along with many numerical checks are illustratd in Sec. C. Finally the growth rates are presentd in Sec. D; in Sec. D.1 by using the dispersion functional $I$, and by using the second formalism in Sec. D.2. Future work is mentioned in Sec. E. 


\section{A. Scalings of Variables for Numerical Purposes}

For the sake of computation, we express the equations of wition as simply as possible by elimiriating as many coefficients of the terms in the equations as we can. To obtain a consistent transformation of variables we start with a Lagrangian L of a charged particle in our VF equilibria.

In CGS units,

$$
L=\frac{1}{2} m \dot{x}^{2}+\frac{1}{2} m \dot{y} \dot{c}+\frac{1}{2} m \dot{z}^{2}+\frac{e}{c} A_{0} \dot{z}-e \Phi_{0}
$$

where the dots indisate the derivatives with respect to time $t$,

$$
\text { e } \Phi_{0}=-T \ln \left(1-\varepsilon_{d} \phi\right) \text { where } \phi=\varepsilon^{2} x^{2}+y^{2}
$$

and the vector potential $\underline{A}_{0}=A_{0} \hat{z}$ with $A_{0}=\frac{B}{2 a} \phi$. Here $B_{a}$ is the magnetic field strength at $x=0$ and $y=a$.

This Lagrangian does not allow us to transform $P_{2}$ away as opposed to the case in Chap. III, because the electrostatic potential does not scale with $P_{Z}$. Yet, the parameter study on the size of plasma can be eliminated by scaling the length in terms of the plasma size. We thoose the scaling so that the minor radius of the separatrix is 1 . Consequently the major radius is $\epsilon^{-1}$. That is $(x, y, z)=a(\tilde{x}, \tilde{y}, \tilde{z})$, where $a$ is the minor radius of the separatrix and the tildes denote the new variables. Hereafter all the new variables will be denoted with tildes unless utherwise roted. The scaling on $\varepsilon_{d}$ then can be 
read off from the argument of the logarithm of $\overline{i q} .(7.2)$ as $\tilde{\mathrm{z}}_{d}=$ $a^{2} \varepsilon_{d}$ since $\phi=a^{2} \tilde{\phi}$. Now let $\Omega_{0}^{-1}$ be the time scaling parameter and $n$ be the average kinetic energy (temperature) scaling parameter so that

$$
t=\tilde{t} / \Omega_{0} \quad \text { and } \quad T=n \tilde{T} \text {. }
$$

Since the equations of motion are independent of a constant factor of $L$, dividing the Lagrangian by $\mathrm{a}^{2} \Omega_{0}^{2}$, we obtain a new Lagrangian $\tilde{L}$.

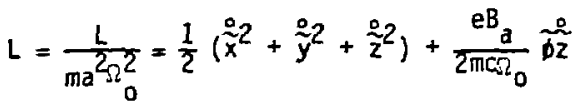

$$
\begin{aligned}
& +\frac{\eta}{m a^{2} \Omega_{0}^{2}} \tilde{T} \ln \left(1-\tilde{\varepsilon}_{d} \tilde{\phi}\right)
\end{aligned}
$$

where ${ }^{\circ}$ denotes the derivative with respect to the new time variable $\tilde{t}$. $\tilde{L}$ can be further simplified by choosing

$$
\Omega_{0}=\frac{e}{m c}\left(\frac{B_{a}}{2}\right) \quad \text { and } \quad n=m \Omega_{0}^{2} a^{2}
$$

to obtain

$$
\tilde{L}=\frac{1}{2}\left(\dot{\widetilde{x}}^{2}+\tilde{\tilde{y}}^{2}+\tilde{z}^{2}\right)+\tilde{\phi}^{0}+\tilde{T} \ln \left(1-\tilde{\varepsilon} d^{\Phi}\right) .
$$


The scalings $(7.4)$ show that

$$
t=\Omega_{0}^{-1} \tilde{t} \quad, \quad T=m a^{2} \Omega_{0}^{2} T \quad \text { and } L=m a^{2} \Omega_{0}^{2} L
$$

Notice that all the new quantities are dimensioniess: time is measured in terms of twice the local gyro time, the $k$ inetic energy (temperature) and :e Lagrangian are measured in terms of twice the local gyro kinetic energy at $x=0$ and $y=a$.

Now, the Euler-Lagrange equation with respect to $\tilde{x}$ gives the equation of motion in $x$ in the new coordinates.

$$
\stackrel{\infty}{\widetilde{x}}=2 \varepsilon^{2} \tilde{x} \tilde{z}-\tilde{T} \frac{2 \tilde{\varepsilon}_{d} \varepsilon^{2} \tilde{x}}{1-\widetilde{\varepsilon}_{d}^{\tilde{\phi}}},
$$

and the cannonical momentum is defined to be

$$
\tilde{P}_{x}=\frac{\partial \tilde{L}}{\partial \frac{\partial}{\tilde{x}}}=\stackrel{\dot{x}}{x} .
$$

Other componencs of the equations of motion are obtained similarly.

Since force is the time derivative of momentum, it is clear that the right hand side of Eq. (7.7) is the force in the new coordinate $\tilde{F}_{x}$. Equating the RHS of Eq. (7.7) with $\underline{\underline{E}}+\underline{\tilde{v}} \times \underline{\tilde{B}}$ so that $\underline{\tilde{F}}=\underline{\underline{E}}+\underline{\tilde{v}} \times \tilde{B}$ $(\underline{\tilde{E}}$ and $\underline{\tilde{B}}$ are dimension less by this definition since $\tilde{F}$ is dimensionless) we obtain 


$$
\tilde{E}_{x}=-2 \frac{\tilde{T}_{e_{d} \varepsilon^{2} \tilde{x}}}{1-\varepsilon_{d}} \quad \text { and } \quad \tilde{B}_{y}=2 \varepsilon^{2} \tilde{x}
$$

which can be compared with the old expression of $E$ and $B$ to obtain

$$
\underline{E}=\frac{m R_{0}^{2} a}{e} \underline{E} \quad \text { and } \quad \underline{B}=\frac{B_{a}}{2} \underline{\tilde{B}} \text {. }
$$

From the force balance equation, and by chossing $n=\frac{m c^{2}}{e^{2} a^{2}} \tilde{n}$, the relation between the pressure gradient and the magnetic field gradient is;

$\tilde{n \varepsilon_{d}} \tilde{T}=\frac{\varepsilon^{2}+1}{2 \pi}$

The new Hamiltonian $\tilde{H}$ corresponding to $\tilde{L}$ is

$$
\tilde{H}=\frac{1}{2} \tilde{P}_{x}^{2}+\frac{1}{2} \tilde{P}_{y}^{2}+\frac{1}{2}\left(\tilde{P}_{z}-\tilde{\phi}\right)^{2}-\tilde{T} \ln \left(1-\tilde{\varepsilon}_{d} \tilde{\phi}\right) \text {, }
$$

and thus scales the same way as $L$ :

$$
H=m R_{0}^{2} a^{2} \tilde{H}
$$

Finally, we have to scale the ion distribution function. We demand $\tilde{f}_{0}$ to be 


$$
\int \tilde{f}_{0} d \underline{\underline{n}} \equiv \tilde{n}_{0}(\tilde{x}) \text {, }
$$

while keeping the same functional dependence of $f_{0}$. Then

$$
\tilde{f}_{0}=\tilde{n}_{0}(0)\left(\frac{1}{2 \pi \tilde{T}}\right)^{3 / 2} e^{-\tilde{H} / \tilde{T}}
$$

and so

$$
\tilde{f}_{0}=\frac{\tilde{n}_{0}(0)}{n_{0}(0)}\left(\frac{T}{\tilde{T}}\right)^{3 / 2} m^{-3 / 2} f_{0}=\frac{e^{2} a^{5} \Omega_{0}^{3}}{m c^{2}} f_{0}
$$

If we summarize the transformation between the ald and the new variables, they are

$$
\begin{aligned}
& (x, y, z)=a(\tilde{x}, \tilde{y}, \tilde{z}) ; k=\tilde{k} / a ; \varepsilon_{d}=\tilde{\varepsilon}_{d} / a^{2}, \\
& t=\tilde{t} / \Omega_{0}, \\
& T=m a^{2} \Omega_{0}^{2} \tilde{T}, \\
& L=m a^{2} \Omega_{0}^{2} \tilde{L}, \\
& H=m a^{2} \Omega_{0}^{2} \tilde{H}, \\
& \underline{E}=\frac{m \Omega_{0}^{2} a}{e} \underline{\tilde{E}}, \\
& \underline{E}=m \Omega_{0}^{2} a \tilde{F}, \\
& \underline{B}=\frac{B_{a}}{2} \underline{B},
\end{aligned}
$$




$$
\begin{aligned}
& \underline{p}=m a \Omega_{0} \tilde{p}, \\
& \underline{v}=a \Omega_{0} \tilde{v}, \\
& n=\frac{m c^{2}}{e^{2} a^{2}} \tilde{n}, \text { and } f_{0}=\frac{m c^{2}}{e^{2} a^{5} \Omega_{0}^{3}} \tilde{f}_{0} .
\end{aligned}
$$

The equilibrium condition gives a relation between $\tilde{n}_{0}(\underline{x})$ and $\tilde{T}$. In our case it is

$$
\tilde{n}_{0}(0) \tilde{\varepsilon}_{d} \tilde{T}=\frac{\varepsilon^{2}+1}{2 \pi}\left(=\frac{1}{2 \pi} \quad \text { for } \varepsilon \ll 1\right) \text {. }
$$

Therefore the dispersion functional is parameterized by $\widetilde{k}$ and $\tilde{\mathrm{T}}$ only. In other words the expectation value of $\tilde{w}$ depends only on $\tilde{k}$ and $\tilde{T}$. This scaling clearly saves us a lot of computational work in the parameter study. For example, evaluation of $\tilde{\tilde{u}}$ for one value of $\tilde{T}$ al lows us to estimate $\tilde{\omega}$ 's of physical systems with different values of $a, \tilde{T}$, $B_{a}$ and $m$ as long as their combination gives the same value $\tilde{T}$ by the following scaling:

$$
\tilde{T}=\frac{T}{m s_{0}^{2} a^{2}}=\frac{4 m c^{2} T}{e^{2} B_{a}^{2} a^{2}}=\frac{T(200 \mathrm{eV}) m(2 M p)}{[a(c m)]^{2}\left[B_{a}(4 K G)\right]^{2}},
$$

where $a$ is the minor radius of the separatrix, $\Omega_{0}=\frac{e B_{a}}{2 m c}$ is half the local gyro-frequency at $x=0$ and $y=a$ and $M p$ is the proton mass. 
In terms of the new variables the VF dispersion functional is

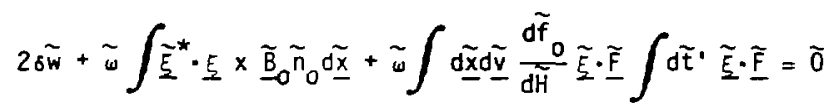

where

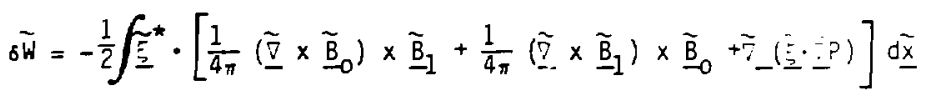

For our equilibrium

$$
\begin{aligned}
& \tilde{f}_{0}(\tilde{\underline{x}}, \underline{v})=\tilde{n}_{0}(0)\left(\frac{1}{2 \pi \tilde{T}}\right)^{3 / 2} e^{\frac{\tilde{H}(\underline{\tilde{x}}, \underline{v})}{\tilde{T}}} \\
& \tilde{n}_{0}(\underline{\underline{x}})=\tilde{n}_{0}(0)\left(1-\tilde{E}_{a} \tilde{\phi}\right),
\end{aligned}
$$

and

$$
\tilde{F}=\underline{\tilde{E}}+\underline{\tilde{v}} \times \underline{\tilde{B}} \text {. }
$$

Now we evaluate each of the terms of the dispersion functional for our trial functions.

$$
\begin{aligned}
& \tilde{s \tilde{W}}=\frac{\varepsilon^{2}}{2 \pi} \int \tilde{d \underline{x}}\left(-\left|\tilde{\xi}_{x}\right|^{2}+\left|\tilde{\xi}_{z}\right|^{2}\right) \\
& \tilde{\omega} \tilde{I}_{c}=\frac{-\varepsilon}{2 \pi \tilde{\varepsilon}_{d} \tilde{T}} \stackrel{\tilde{\omega}}{\tilde{k}} \int\left|\tilde{\xi}_{x}\right|^{2}\left(1-2 \varepsilon_{i} \tilde{\phi}\right) d \underline{x}
\end{aligned}
$$


and

$$
\begin{aligned}
& \left.\tilde{I}_{o b}=i \tilde{\omega}_{0} ; 0\right)\left(\frac{1}{2 \pi \tilde{T}}\right)^{3 / 2}\left(-\frac{1}{\tilde{T}}\right) \int d \underline{x} d \tilde{v} e^{-\tilde{H} / \tilde{T}_{\tilde{\xi}}^{*}} \cdot \tilde{F} \int d t \cdot \tilde{\xi} \cdot \tilde{F} \\
& =-\frac{\sqrt{2 \pi}}{\varepsilon \tilde{T}^{7 / 2}} \stackrel{\tilde{\omega}}{\widetilde{k}} \int d B d \tilde{H} \int d \tilde{P}_{Z} \frac{e^{-\tilde{H} / \widetilde{\bar{T}}\left(\tilde{\bar{B}} ; \tilde{H}, \widetilde{P}_{Z}\right)}}{\widetilde{\beta}-\tilde{\omega}} \\
& =-\frac{\sqrt{2 \pi}}{\varepsilon \tilde{T}^{T / 2}} \frac{\tilde{\omega}}{\tilde{k}} \int d \tilde{B} \frac{\tilde{S}(\tilde{B})}{\tilde{B}-\tilde{\omega}}
\end{aligned}
$$

where $\mathcal{P}\left(\tilde{B} ; \tilde{H}, \tilde{P}_{Z}\right)$ is the dynamical spectral densities of $\tilde{\tilde{\xi}} \cdot \widetilde{F}$ at $\widetilde{H}$ and $\tilde{\mathrm{P}}_{z}$. Finally, the dispersion functionai in our dimensionless units is the following dropping the tilde notation,

$$
\begin{aligned}
\int d \phi\left[-\xi_{x}^{2}+\xi_{z}^{2}\right] & +\omega\left[-\frac{1}{\varepsilon_{d} T k}\right] \int d \phi\left(1-2 \varepsilon_{d} \phi\right)\left|\xi_{x}\right|^{2} \\
& +\omega\left[-\frac{1}{2 \sqrt{2 \pi} \varepsilon^{2} \varepsilon_{d} T^{T / 2}}\right] \int d \beta \frac{S(\beta)}{B-\omega}=0
\end{aligned}
$$

where

$$
S(B) \equiv \int d H d P z e^{-H / T} \int_{\phi_{m n}}^{\phi_{m x}} d \phi k^{\star}(z(t)) K\left(z\left(t^{\prime}\right)\right)
$$

and

$$
K(\underline{z}(t)) \equiv \underline{\xi}(\phi) e^{i k z} \cdot\left(\underline{E}_{0}+\underline{v} \times \underline{B}\right)
$$


From Eq. (7.23) we see that there are only two parameters $T$ and $k$ which determines w for a chosen trial function. $T$ is the squared quantity of the ratio of gyro-radius to the size of the plasma and $k$ is equal to the toroidal mode number if the cylinder were "rolled-up" into a compact torus of major radius a. The inertia term is evaluated by

$$
\begin{aligned}
K_{0} \equiv \text { inertia term } & =\frac{1}{2} n \int\left(1-\varepsilon_{d}^{\phi}\right)\left(\xi_{x}^{2}+\xi_{z}^{2}\right) d \underline{x} \\
& =\frac{1}{\pi \varepsilon_{d}^{\top}} \int\left(1-\varepsilon_{d} \phi\right)\left(\xi_{x}^{2}+\xi_{z}^{2}\right) d \underline{x}
\end{aligned}
$$

and so the MHD growth rạtes are

$$
r_{M H D}^{2}=-2 \varepsilon^{2} \varepsilon_{d}^{\top} \quad \frac{\int d \phi\left(-\xi_{x}^{2}+\xi_{z}^{2}\right)}{\int\left(1-\varepsilon_{d} \phi\right)\left(\xi_{x}^{2}+\xi_{z}^{2}\right) d \phi} .
$$

The dispersion functional of the second expression is from Eq. (4.31b)

$$
\begin{aligned}
2 \delta W-2 \omega^{2} K_{0}+\omega\left[\int \frac{S_{1}}{B-\omega} d B\right. & +\omega\left(\int \frac{S_{2}}{B-\omega} d B+\left(\int \frac{S_{2}}{B-\omega} d B\right)^{*}\right) \\
& \left.+\omega^{2} \int \frac{S_{3}}{B-\omega} d B\right]=0
\end{aligned}
$$

where $S_{1}$ and $S_{3}$ are obtained exactly the same way as $S$ in Eq. (6.25) by simply replacing $\underline{\xi} \cdot \underline{F}$ by $\underline{v v}: \underline{\nabla} \underline{\underline{F}}$ and $\underline{v} \underline{\underline{\xi}}$ and the conjugates respectively. $S_{2}$ is obtained by replacing $(\underline{\xi} \cdot \underline{F}) *$ by $(\underline{v v}: \underline{\nabla \xi}) *$ and $\underline{\xi} \cdot \underline{F}$ by $\underline{v} \cdot \underline{\xi}$. 


\section{B. STABILITY MECHANISMS}

The source of MHD instability is the bad curvature of the magnetic field lines with respect to the pressure gradient. The $\nabla B$ relative to the pressure gradient is in fact favorable in this geometry, but the net effect of $\underline{\nabla B}$ and the curvature is unfavorable for stability. This kind of instability can be understood as Rayleigh-Taylor instability by taking the combined effect of $\underline{\nabla B}$ and curvature as an effective gravity acting on the plasma.

The Rayleigh-Taylor instability arises when the gravity and the density gradient are such that, once there appears a charge separation by a perturbation, then the charge separation develops more and more. These instabilities can not exist if the separated charges are swept out by some mechanism faster than the predicted MHD growth time. Figure 7.1 shows the MHD $\mathrm{m}=1$ modes. These modes can not exist if oppositely charged regions smear out either along the cylindrical axis or across the major axis of the ellipses of the flux contours. The minor radius is excluded since the instability can stil? develop at the tips of the ellipses even if the charge separation across the minor axis is smeared out (ballooning modes). Since twice the major radius is $2 / \varepsilon$ and the wave length in the axial direction is $\pi / k$ the distance of charge separation is approximately $\pi / k$ for all $k$ $(k=1,2,3, \ldots)$. (Notice that $k$ here is the new variable where the tilde on top is suppressed, while the $k$ of Fig. 7.1 is an old variable.) 
If the orbits are big (bigger than the length of the charge separation), the communication time (time sor smearing the charge separation) is the length of the charge separation devided by the thermal velocity. This happens approximately when $T=\pi^{2}=10$. If the orbits are small then the thermal velocity should be replaced by the drift velocity. Notice that there is no fluid drift in our model, but the individual particles drift along the cylindrical axis. In our dimensionless units, the exponentiation MHD growth time is $1 / Y_{M H D}=1 /(0.1 \sqrt{T})$ for trial function I, and the connection tinie is $\pi /\left(k v_{t h}\right)=\pi /(k \sqrt{T})$ for the large orbits, and $\pi /\left(k v_{d}\right)=\pi /(k T)$ for small orbits. Therefore, we expect that the instability does not exist if $k \geq 0.2$ for large orbits $(T \geq 10)$, and $k \geq 0.3 / \sqrt{\top}$ for smali orbits $(T \geq 10)$.

The orbit effects can be seen from the VF dispersion functional. The orbit cerrection to MHD is $\omega \int \frac{S_{1}}{B-\omega} d B$ from the second formalism of the dispersion functional where $S_{1}$ is the two time correlation of

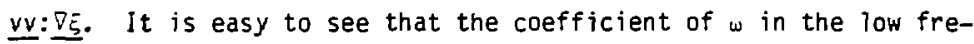
quency limit is increasing as $k T$ or $T / a$ does.

\section{Stochastic Effect}

implementation of finite orbit brings resonance effect of particles with the wave. It can either make the wave grow or damp away. Stochastic orbits can not remain in resonance with the wave, and some of the possible resonance modes may be suppressed in the presence of stochastic particles. 
C. Numerical Checks and Some Intermediate Steps

We present in this section some of the numerical checks that are made to test the computer code and some numerical tests made to see whether our assumptions valid. Also, the intermediate steps of finding the spectral densities are presented since there are many steps involved.

\section{C.1 Transformation of Variables}

In $\underline{x}$ and $\underline{v}$ variables

$$
\int \underline{d x d} \underline{v} f_{0}(\underline{x}, \underline{v})-\int d \underline{x} d \underline{v} e^{-\left(v^{2} / 2+e \Phi(\underline{x}) / T\right.}-T^{-3 / 2}
$$

In terms of $(H, P, \theta, \phi, \theta, z)$

$$
\begin{aligned}
\int d \underline{x} \underline{v} f_{0}(\underline{x}, \underline{v}) \rightarrow \int d \phi d \theta d z & \int_{-\infty}^{\infty} d H d P_{z} d \theta e^{-H / T} \delta\left(H-\frac{v^{2}}{2}-e \phi(\underline{x})\right) \delta\left(P_{z}-\alpha \phi\right) \\
& -\int_{a} d H d \underline{z} \int_{\phi_{m n}}^{\phi} d \phi e^{-\tilde{H} / \tilde{T}}
\end{aligned}
$$

whei- the subscript a of the integral sign denotes that the integral is over only for the allowable region shown in Fig. 6.2. Evaluation of the last term of Eq. (7.25) numerically on a $5 \times 5$ grid values in $\mathrm{H}-\mathrm{P}_{\mathbf{Z}}$ space shows the dependence on $\mathrm{T}$ very accurately. 


\section{C.2 Two Time Correlation of $v_{y}$}

Figure 7.2 shows correlation functions and spectrum of a regular orbit.

Plot (a) is a two time correlation of $v_{y^{*}}$ In fact, if there were no windows applied, the emplitude would not be decreasing. Nevertheless, the spectrum in plot (b) which is the time Fourier transformation of plot (a) has a form of modulation of frequencies $\omega_{1}$ and $\omega_{2}$ with a window function $w f(\tau): w f(\tau) \cos \omega_{1} \cos \omega_{2}$ with $\omega_{1}=10 \omega_{2}$. A window function is a function of to be multiplied by another function of to select only the frequencies of interest upon time Fourier transformation or to eliminate the numerical errors. We use window functions to eliminate unphysical high frequencies. The spectrum in (b) has peaks at $(1 / 2) \cdot\left(\omega_{1} \pm \omega_{2}\right)$ properly. Only the positive frequencies are shown since the spectrum obtained from the correlation of a real function is symmetric in frequency. The role of windows is to force the records to be periodic, to get rid of the unphysical high frequency spectra which arise when the records are not periodic. The artificially added windows do not change the spectrai frequencies but only their width. The width can be adjusted as we want by giving proper windows and by obtaining correlations for a longer time interval.

Plot (c) is the average correlation of 50 particles. Due to the initial thase average, the corrolation naturally decays (almost exponentially) and the spectrum shown in (d) fills up certain range of frequencies, thus the spectrum becomes fatter. 
Figure 7.3 shows the case of stochastic orbits. Plot (a) is the $x y$ trajectory where $(b)$ and $(c)$ are the two time correlation of $v_{y}$ and the spectral densities of the motion (a) respectively. One more example of stochastic motion is shown from (d) to (f) with the same sequence as in (a) to (c). It is not easy to tell whether the motion (d) is stochastic or not, but the surface of section plot shows that the motion is stochastic. Both (b) and (e) show almost exponentially decaying correlation. Both (c) and ( $f$ ) show the stochastic broadening of the spectral densities. Therefore, the spectral conditions can be treated similarly both for regular and stochastic orbits.

\section{3 Is Phase Average the Same as Time Average for Stochastic Orbits}

From Eq. (6.30) the question is equivalent to whether the following equality holds when many initial conditions are used:

$$
\begin{aligned}
& \int d \underline{\mathcal{T}} \mathcal{K}\left(z_{z}\left(t_{0}\right), \tau\right)=\frac{\mathcal{U}\left(H, P_{z}\right)}{N} \sum_{i=1}^{N}\left\langle\mathcal{K}\left(z_{i}(t), \tau\right)\right\rangle_{t} \\
& \partial \Gamma\left(t_{0}\right)
\end{aligned}
$$

The above equation involves three averaging procedures: If we devide the equation by $Q\left(H, P_{z}\right)$, the left hand side is the average

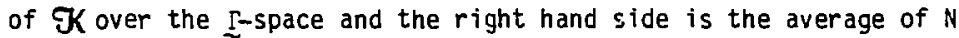
initial conditions of the time-averaged $\mathfrak{K}$.

To answer the question we evaluate both sides of the above equation numerically. The results are iliustrated in Fig. 7.4. The left hand side of the Eq. $(7.29)$ is evaluated by taking 100 initial points randomly in space and is shown in plot (a), while the right hand 
side of the Eq. (7.29) is obtained for 20 particles and shown in plot (c). When we choose more particles for the straight-forward pnase integral the amplitude of the correlation decays to a smaller value, which is reasonable since the fluctuation of the correlation at large t becomes small by averaging over more particles. This produces a better spectrum near zero frequency and the negative spectral densities (which are purely numerical errors) are reduced.

The amplitudes of the correlations of plot (a) and (c) are not the same, but similar. Furthermore the spectral densities shown in plat (b) and (d), which are the time fourier transformation of plot (a) and (c) respectively, are very much the same. Both of the spectral densities are obtained by applying log windows to elliminate the unphysica? high frequency noise.

\section{4 Is the Dependence of the Third Invariant of our Correlation Weak} for Regular Orbits

To make the orbit calculation practical for regular orbits, we made the strong assumption that the correlation function is weakly depend on the third invariant. Since $H$ and $P_{z}$ are the exact invariants in our geometry, the third invariant, whatever it is, will make the orbits regular.

The question is

$$
\frac{Q\left(H, P_{2}\right)}{N_{1}} \sum_{i=1}^{N_{1}} \Im K_{i}=\frac{Q\left(H, P_{2}\right)}{N_{2}} \quad \sum_{j=1}^{N_{2}} \Im K_{j}{ }^{\prime} t
$$


Thus, if the equality

$$
\left.\frac{1}{N_{1}} \sum_{j=1}^{N_{1}} \mathfrak{K}_{i}=\frac{1}{N_{2}} \sum_{j=1}^{N_{2}} \mathcal{J K}_{j}\right\rangle_{t}
$$

holds approximately, the assumpton is valid, which is the same condition as Eq. (7.29). Therefore, to test the validity of the assumption we evaluate exactly the same quantities of Fig. 7.4 for reguiar orbits. The results shown in Fig. 7.5 are qualitatively the same as the stochastic case. Thus, the assumption that our correlation depends on the third invariant weakly for regular orbits is valid. Therefore we can treat the phase integral by time integral for all kinds of orbits, both regular and stochastic. The saving in computation time by transforming the phase average to the time average is enormous.

\section{C.5 Dynamical Spectral Densities $\Phi(B: H, P=2$}

The dynamical spectral densities $S(B)$ of a system are evaluated by summing the spectral densities of $P\left(B: H, P_{Z}\right)$ over $H$ and $P_{Z}$ with an appropriatr: weighting (Refer Eq. (6.25)). Therefore we first investigate $P\left(B: H, P_{z}\right)$ for given $H$ and $P_{z}$ values.

We choose a system of $\varepsilon=0.1$ and $T=0.1$, and study the case of $k=10$. Figure 7.6 shows five spectral densities of $\Phi(B: H, P)$, whose $H$ and $P_{Z}$ values correspond to those of five in $F i g .6 .2$ from (a) through (e) respectively. The peaks of the plots occur approximately at $v_{d}$ where $v_{d}=p-\langle\phi\rangle$. $v_{d}$ changes sign from (a) to (e), 
thus the sign of the peaks also change. The width of the spectrum depends on three different effects; thermal spread (randomness in initial velocity), stochasticity of the orbits and the harmonics of the oscillation frequencies, Particles of plot (a) are $J_{y}$-conserving regular, thus the width in this case is not due to the stochasticity. Whether the width attributed to the randomeness in velocity and/or the natural frequencies of the motion can be tested in comraring the spectrum of a single orbit and that of many orbits. The plot 'a) is the spectrum for 10 particles which shows a very good ensemble average (taking many more particles makes little change to the spectra. densities). When only one particle is used, the spectral densities are not that different at a large scale. This indicates that it is the particle frequencies that is mainly responsible for the width of the spectrum. This are one of the reasons that using only a few pa:-icles is sufficient to obtain a good picture of the spectrum. What effects are more important for the width of the spectrum depends on $\mathrm{H}_{\text {and }} \mathrm{P}_{2}$. Particles shown in (d) are mostly stochastic. Here we see wider spectral spread mainly due to the stochasticity. Sum of the five spectral densities from (a) to $(e)$ are shown in plot (f). Suming over values corresponds to the randomness in $v_{z}$.

For different $H$ values, the dependence of $P_{z}$ is qualitatively the same except that the frequencies anpear at higher values for higher values of $H$ due to the fast particle motion.

Finally, the spectral densities $S(B)$ is obtained after summing over five different $H$ values (randomness in magnitude of velocity) with proper weighting, and this is shown in Fig. $7.7(a)$. 


\section{RESULT AND DISCUSSION}

\subsection{By Using the 1st VF Dispersion Fun . ional}

From $S(B)$ of Fig. $7.7(a)$ the expectation value of $\omega$ of Eq. (6.43) is evaluated first by making a Taylor expansion around $w=0$. After evaluating all the coefficients as explained in Sec. E.4 of Chap. VI, we obtained the following for $T=0.1$ and $k=10$.

$$
-0.195-3.09 \times 10^{-2} \omega^{2}-0.258 \omega-i \pi S(0)=0 .
$$

which is to be compared with

$$
-0.195-113 \omega^{2}=0
$$

of MHD dispersion relation. Comparing the coefficients of $\omega^{2}$ of the above two dispersion relations we see that the spectral deniities of Fig. 7.7(a) are not accurizta enough to give the proper inertia term. There are two possibilities for that matior: one is that we did not follow the orbits long enough to get accurate spectral densities near zero frequency. The other is that higher order of w's are important. The latter possibility is tested by drawing Nyquist Diagram explained in Sec. E.3 in Chap. IV.

Shown in Fig. $7.7(\mathrm{~b})$ is a large scale diagram and an enlarged diagram around the crossing point is shown in (c). The diagrams of iarger growth rates encircle the dia $\mathbf{a}_{\text {- }}$-ams of the smaller values of the growth rates. The inner curve of plot $(c)$ is for $\gamma^{2}=10^{-3}$ and the outer is 
for $r^{2}=10^{-4}$. Therefore the crossing $\left(-2 \delta \mathrm{W}=0.195,-I_{c}=0.193\right)$ occurs for $10^{-3}<r^{2}<10^{-4}$. Therefore the growth rate is not much reduced. Exact calculation evaluated without making Talor expansion and a good initial guess $w$ value indicates that the growth rate is reduced only a factor of two or three.

This phenomenon appears for almost all parameter values that we tested. The reason is that the real part of the frequency is always smaller than the smallest frequency that is accurately obtained by following the orbits for about MHD growth time ( $\left.T_{M H D}\right)$ which is $1 / T_{M H D}$ ). (Notice that in the VF model $\sigma \rightarrow 0$ when $r \rightarrow 0$. ) This indicates that we have to obtain spectral densities more accurate?y near zero frequency by following each orbit longer, which we believes resolve the problem.

\subsection{By Using the 2nd Formalism of the VF Dispersion Functional}

Since the 2 nd formalism gives the kinetic correction more explicitly, first we investigate the stability by following the orbits only up to the order of MHD growth time as in Sec. A.

We use the same procedure as in Sec. A for the spectrum of $\underline{v V}: \underline{\nabla \xi}$. Qualitatively the same comments can be made for the dependences of $H$ and $P_{z}$. Therefore we show only the final spectral densities in Fig. $7.8(a)$ for the case of $\varepsilon=0.1, T=0.1$ and $k=10$, which are the same values as in Sec. A.

As before, first we make a Taylor expansion in w of the 2nd formalism to get an approximated expectation values of $\omega$. For the spectral densities of Fig. $7.8(a)$ we have 


$$
-0.195-(113+123) \omega^{2}-24.0 \omega-i_{n} S(0)=0 .
$$

which is to be compared with the MHO dispersion relation Eq. (7.33). The solution of Eq. (7.34) is $\omega=\left(-8.9 \times 10^{-3}, 5.2 \times 10^{-4}\right)$ and $\omega=\left(-9.2 \times 10^{-2},-5.4 \times 10^{-3}\right)$ which show a very reduced growth rate $\left(0.01 r_{M H D}\right)$. The solution which gives damping rates, of course, is not physical since the dispersion functional is valid only for growing modes. When a full expression is used without making the Taylor expansion $\omega=\left(-8.9 \times 10^{-3}, 5.3 \times 10^{-4}\right)$ and $\omega=\left(-9.2 \times 10^{-2}\right.$, $-8.3 \times 10^{-3}$ ) which are almost the same as the values evaluated by using the Taylor expanded form. This approximation is found to b: very good for all the cases we tested.

Setting the real part and the imaginary part of the second expression of the dispersion functional (see Eq. 7.26), we obtain a similar expression as Eq. (4.49).

$$
\begin{aligned}
& -2 \delta w-2\left(\sigma+\gamma^{2}\right) K_{0}+\left(\sigma^{2}+\gamma^{2}\right) \int d B \frac{S_{1}}{(\beta-\sigma)^{2}+\gamma^{2}}=0 \\
& 2 K_{0}-\frac{1}{2 \sigma} \int \frac{B S_{1}}{(\beta-\sigma)^{2}+\gamma^{2}}=0
\end{aligned}
$$

Now we define

$$
\text { Real }\left[G_{1}(\omega)\right]=2\left(\sigma^{2}+\gamma^{2}\right) k_{0}-\left(\sigma^{2}+\gamma^{2}\right) \int d \beta \frac{S_{1}}{(\beta-\sigma)^{2}+\gamma^{2}}
$$


and

$\operatorname{Imag}\left[G_{2}(\omega)\right]=\frac{1}{2 \sigma} \int \frac{\beta S_{1}}{(\beta-\sigma)^{2}+\gamma^{2}}$

Here we ignored the terms involving $S_{2}$ and $S_{3}$ in the dispersion functiona $I$.

A Nyquist Diagram is obtained by plotting the Image $\left[G_{1}(\omega)\right]$ on the vertical axis and Rea] $\left[G_{1}(u)\right]$ on the horizontal axis by changing $\sigma$ from $-\infty$ to $+\infty$. We call this the Nyquist Diagram II Since it is for the dispersion functional II.

The expectation value of w can be obtained by observing the value of $w$ when the Diagram passes the point $\left(2 \delta \mathrm{H}, 2 K_{0}\right)$ in $G_{1}(w)$ plane.

The Nyquist Diagram II are plotted in Fig. $7.9(\mathrm{~b})$ for $\gamma^{2}=10^{-2}$ and $10^{-4}$ by taking the spetral densities of $F: g .7 .9(a)$. It indicates that the growth rate is much smaller than $10^{-2}$ which is consistent with the solution of Eq. (7.34).

To show how the spectral densities change with respect to $T$, we present a series of the spectral densitics of $S_{1}(\beta)$ in $r i g .7 .9$ from plot (a) to (d) for the trial function I. The values of $T$ are 10, 0.1 , $10^{-3}$ and $10^{-5}$ respectively. As we expected, the spectral frequencies are higher for higher $T$. The peaks of the spectra appear around $\pm k \sqrt{T}$ except $T=10^{-5}$. For this low $T$, the gyro-frequencies are more responsible for the spectrai structure. For $k=1$, the $T$ dependence on the spectral frequencies holds for large $T$ but the values are not $k v \bar{T}$. When $T$ is small the spectral frequencies ranges are about the same for all the $k$ values chosen, which is because the thermal effect 
is negligible compared to the gyro-frequencies for particls dynamics for low T.

Shown in $(e)-(h)$ are exactly the same quantities for the trial function II. The $T$ dependence is the same as that of the trial function I. Comparing the spectral densities of the two different trial functions, we notice that the spectrum of the second trial function persist to hicher frequencies than those of the first, which is because the second trial function has shorter length scaling than that of the first.

The $k$ dependence on $S_{1}(\beta)$ are shown in Fig. 7.10. Shown is the case of $\varepsilon=0.1$ and $T=0.1$. The values of $k$ are 1 for $p l o t(a), 10$ for plot (b) and 100 for plot (c). For large $k$, the spectral frequencies are proportional to $k$ but not for small $k$ values. In short, if the oscillation frequencies of particles are larger than the value of $k \sqrt{T}$, the spectral frequencies do not scale as $k \sqrt{\top}$, which occurs at low T or low $k$.

Growth rates are obtained for $k=1,10$ and 100 each for $T=10$, $0.1,10^{-3}$ and $10^{-5}$ by using the trial function $I$. For large values of $k(k=100)$ the growth rates are reduced very much $\left(\right.$ to $\left.-10^{-3} Y_{\text {HMD }}\right)$ for large $T$ and approaches to that of MHO for small T. For $k$ about 10 , the growth rates are reduced by factor of 100 or 10 for $T$ around 0.1 and for low $T\left(T=10^{-5}\right)$ they approach to that of MHO. However, the growth rates are reduced only by a factor of 10 for $T$ as large as 10 . Threfore we follow the particles about 10 times longer than before for this case $\left(-10 T_{M H O}\right)$. Then the growth rates are very much reduced 
(10 times or even smaller than that of MHD). For $k=1$ none of them show reduced growth rates. Following the particles 10 times longer did not improve the stabrlity for low $k$ values.

The growth rates are also evaluated by using the trial function II which is localized away from the null. Similar phenomena appear except that the magnitudes of the growth rats are somewhat different. For $k=10$, factor of 10 for $T=0.1$ and remains the same order as that of MHO for $T=10^{-3}$ and $T=10^{-5}$. The growth rates are the same order as those of MHD for all T values tested as for the case of trial function I. The summary of the growth rates of different $k$ and $T$ values are shown in Fig. 7.11 .

If we take the experiments of FRC at LOS Alamos National Laboratory, we obtain $T \sim 0.1$ for $F R X-B$ and $T=.025$ for $F R X-C$ from $E q$. (7.17). The results summarized in Fig. 7.11 indicate that, although a direct comparison cannot be made since our geometry is linear, the plasma is essentially stable against the $m=1$ axial shifting modes for large toroidal mode numbers but not for low toroidal mode numbers. (Again, notice that the tildes for the new variables are suppressed here in the text but not in the figure.)

One may be able to see reduced growth rates for low $k$ values by taking into account one or some of the following factors: (1) We should follow particles a lot longer for these parameters. (2) The term that we neglected become more important for small k values. (3) The toroidal effect should be taken into account. 


\section{E. Future Work}

Some short-term future work is to (1) follow the orbits langer for the dispersion function I for all parameters, and for low $k$ values for the dispersion function I!. (2) Study the effects of the ignored terms in the second dispersion functional for low $\mathrm{k}$.

An intermediate-term work might be to study the toridal effect on the stability. A long-term work is to solve the full dispersion matrix. 


\section{FIGURE CAPTIONS}

Fig. 7.1. MHD internal $m=1$ modes in an elongated Z-pinch. Shown are the two flux tubes; the inner curved one is the perturbed flux surface and the outer one is the separatrix.

Fig. 7.2. Correlation functions and the spectral densities for regular orbits. Plot (a) is the correlation of $v_{y}$ of a regular orbit. The dynamical spectral densities are shown in (b). Plot (c) is the average correlation of 50 randomly chosen particles in the allowable region in $\Gamma$ space. The averaged spectral densities are shown in (d).

Fig. 7.3. A stochastic orbit, the correlation of $v_{y}$ and the spectrum. xy trajectory of a stochastic particle is plotted in (a). The sorrelation of $v_{y}$ is shown in (b) and the spectral densities are in $(c)$.

Fig. 7.4. Correlations and the spectral densities of stochastic orbits. Plot (a) is the phase-averaged correlation over 100 initial points. Plot (c) is the time-averaged correlation-shown is the average of 20 time-averaged correlations. The spectra? Jensities of correlations (a) and (c) are shown in (b) and (d) respectively.

Fig. 7.5. Correlations and the spectral densities of $J_{y}$-conserving orbits. Plot (a) is the ihase average over 200 initial pcints. Plot (c) is 1 erage of 20 time-averaged correlations. Plot (b) and (d) are the spectral densities of the correlations of (c) and (c) respectively. 
Fig. 7.6. Dynamical spectra densities for $\varepsilon=0.1, T=0.1$ and $k=10$. From plot (a) to (e) are the dynamical spectral densities of the five different $P_{Z}$ values of a given $H$. Each plot is the averaged spectrum over 10 different initial conditions. The five values of $\left(H, P_{z}\right)$ are shown in Fig. 6.3 labeled (a) through (e). The plots from (a) to (e) correspond to the spectrum $P\left(B ; H, P_{2}\right)$ of step 3 shown in Eq. (6.25). Plot (f) is the some of the five spectral densities with proper weighting functions (Step 4 of Eq. (6.25).

Fig. 7.7. Spectral densities and the Nyquist Diagram (I). Plot (a) is the final dynamical spectral densities $S(B)$ after summing over all energy values (Step 5 of Eq. (6.25)) for $\varepsilon=0.1, T=0.1$ and $k=10$. Plot $(b)$ is the Nyquist Diagram as a large scale (for $\gamma^{2}=10 ., 1$. and $10^{-3}$ ) and the enlarged diagram around the crossing $(-2 \delta w=0.195$, $\left.-I_{c}=0.193\right)$ is showin in plot $(c)$. The dotted lines are for $\sigma<0$ and the solid lines are for $\sigma>0$.

Fig. 7.8. Spectral densities and the Nyquist Diagram (II). Plot (a) is the final dynamical spectral densities $S_{1}(B)$ which is the correlation of $\underline{v v}: \underline{\nabla \xi}$ for $\varepsilon=0.1, T=0.1$ and $k=10$. Plot (b) is the Nyquist Diagram. The dotted lines are for negative o values and the solid lines are for positive o values. 
Fig. 7.9. Dynamical spectral densities for $\varepsilon=0.1$ and $k=10$. Plot (a) through (d) are the spectral densities for the trial function I for $T=10,0.1,10^{-3}$ and $10^{-5}$ respectively. Plot (e) through (h) are the spectral densities of the trial function 11 for $T=10,0.1,10^{-3}$ and $10^{-5}$.

Fig. 7.10. Dynamical spectral derisities of $T=0.1$ for the trial function I. The spectral densities of $k=1,10$ and 100 are shown in from plot (a) to (c) respectively.

Fig. 7.11. Growth rates vs. T for $k=1,10$ and 100 . For trial function I, cross marks are used far $k=1 \mathrm{u} 0$, solid circles for $k=10$ and solid triangle; are used for $k=1$. For trial function II, open circles are used for $k=10$ and open $t_{1} j_{-}$ angles are used for $k=1$. 


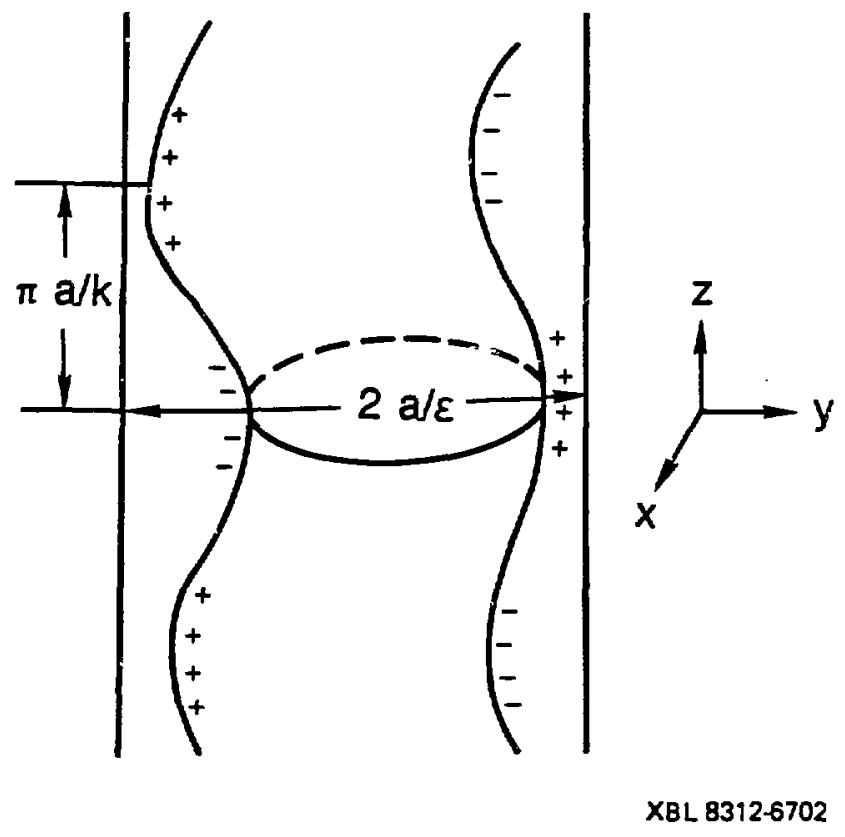

Figure 7.1 

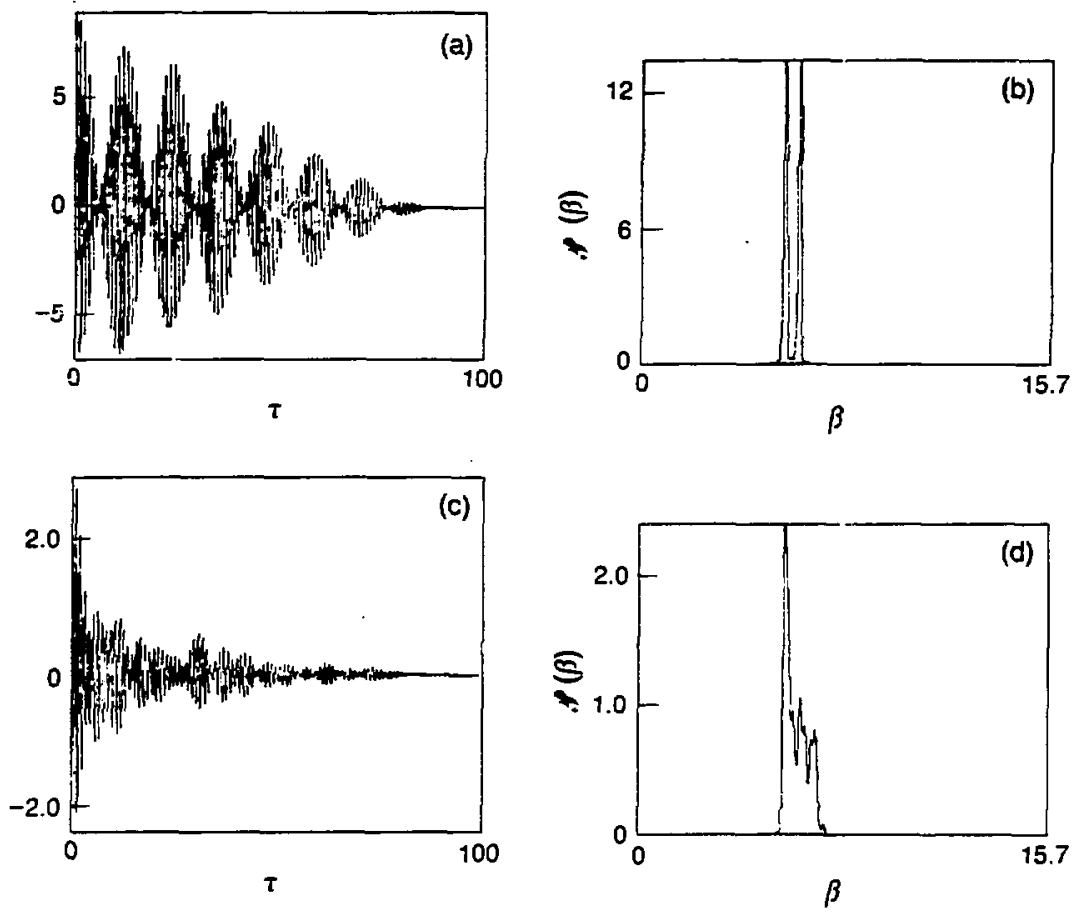

XBL $8312-6700$

Figure 7.2 

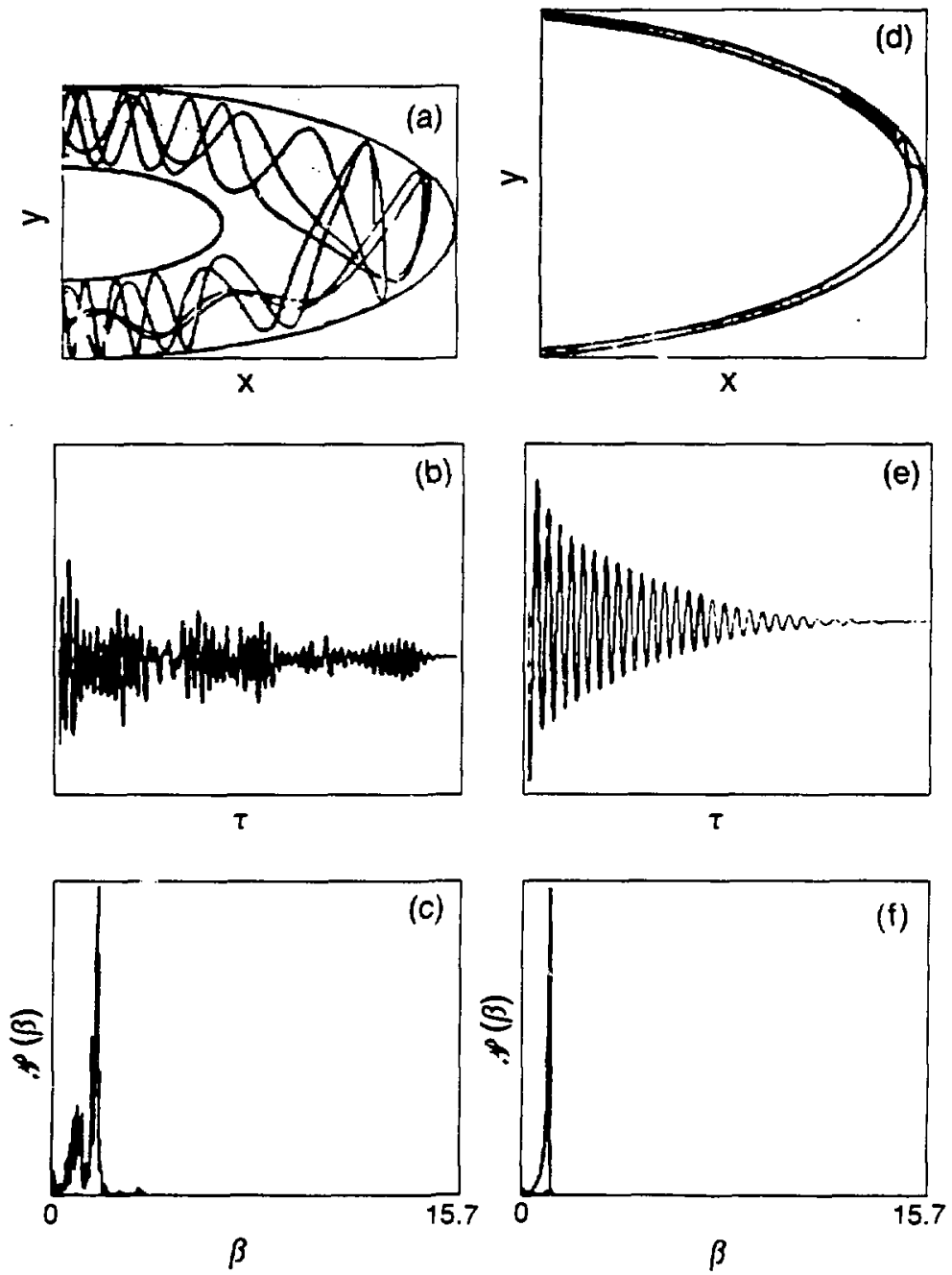

Figure 7.3 

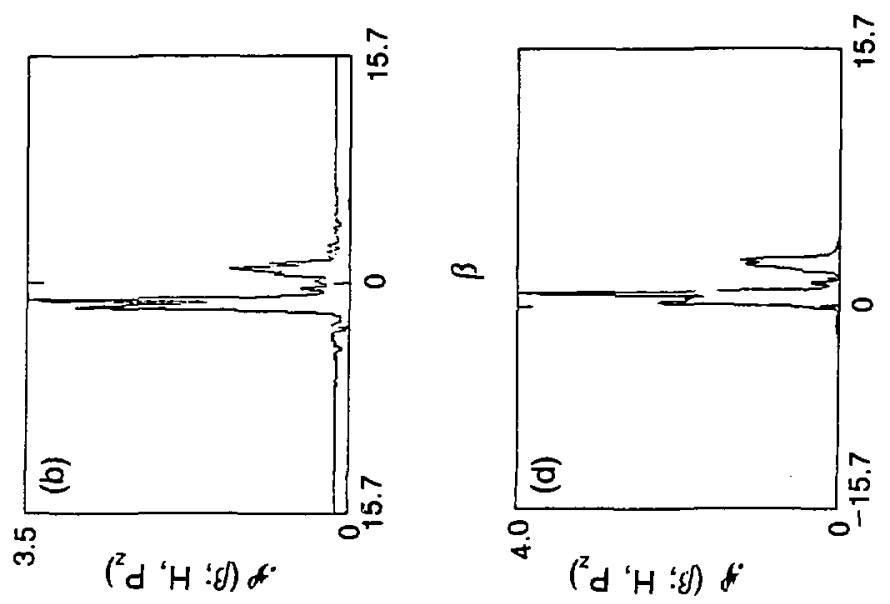

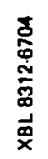
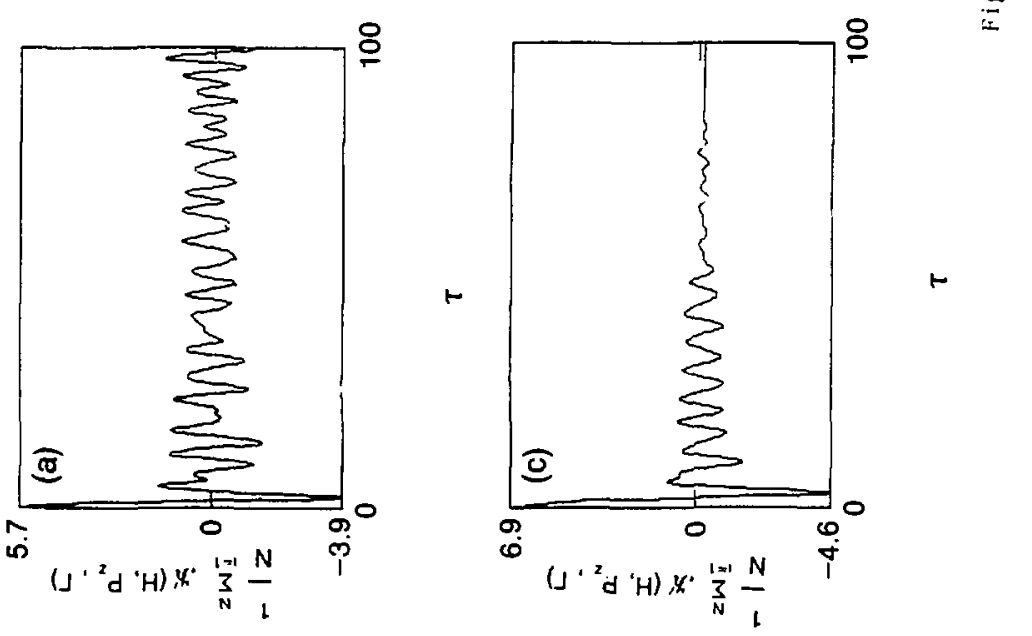

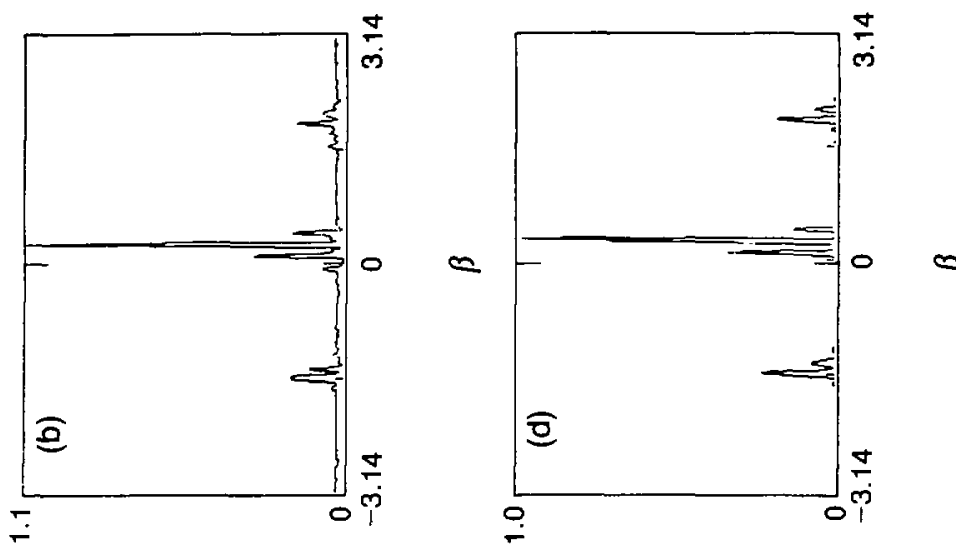

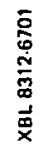
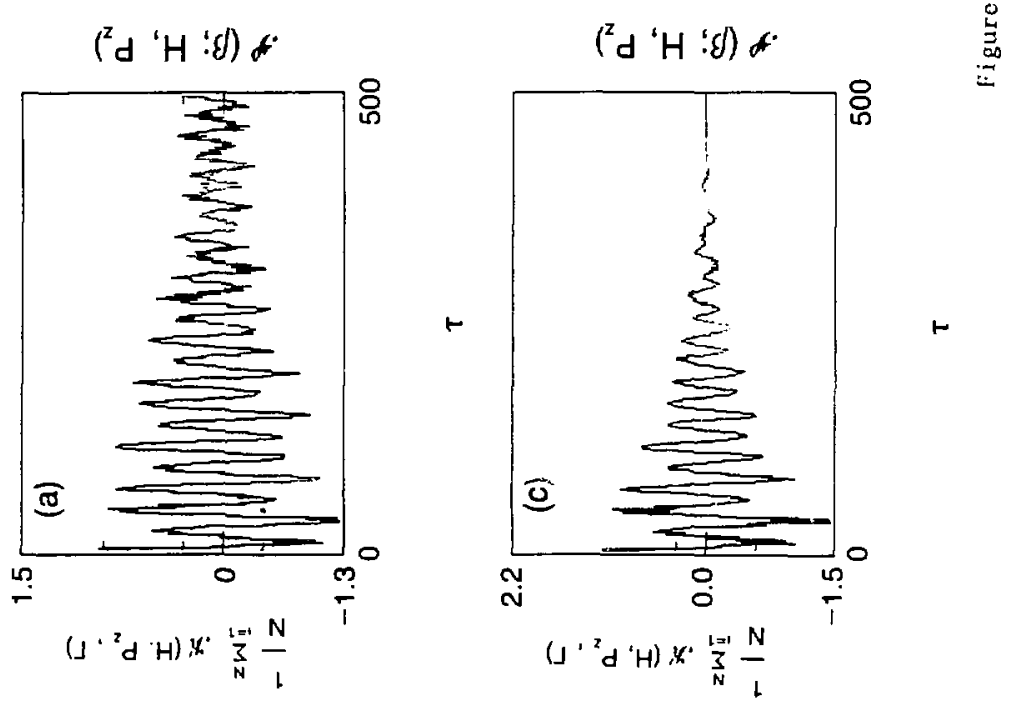


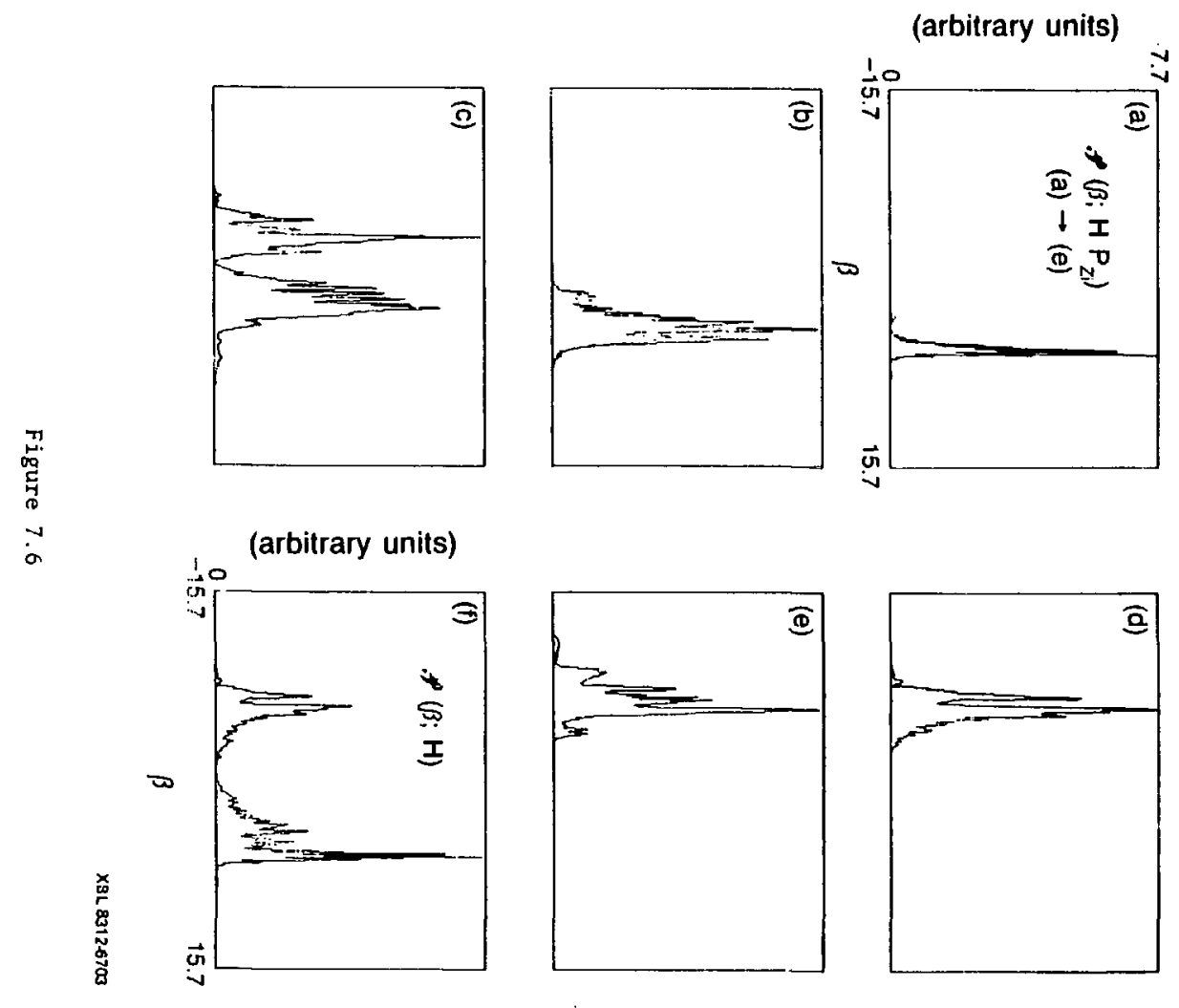



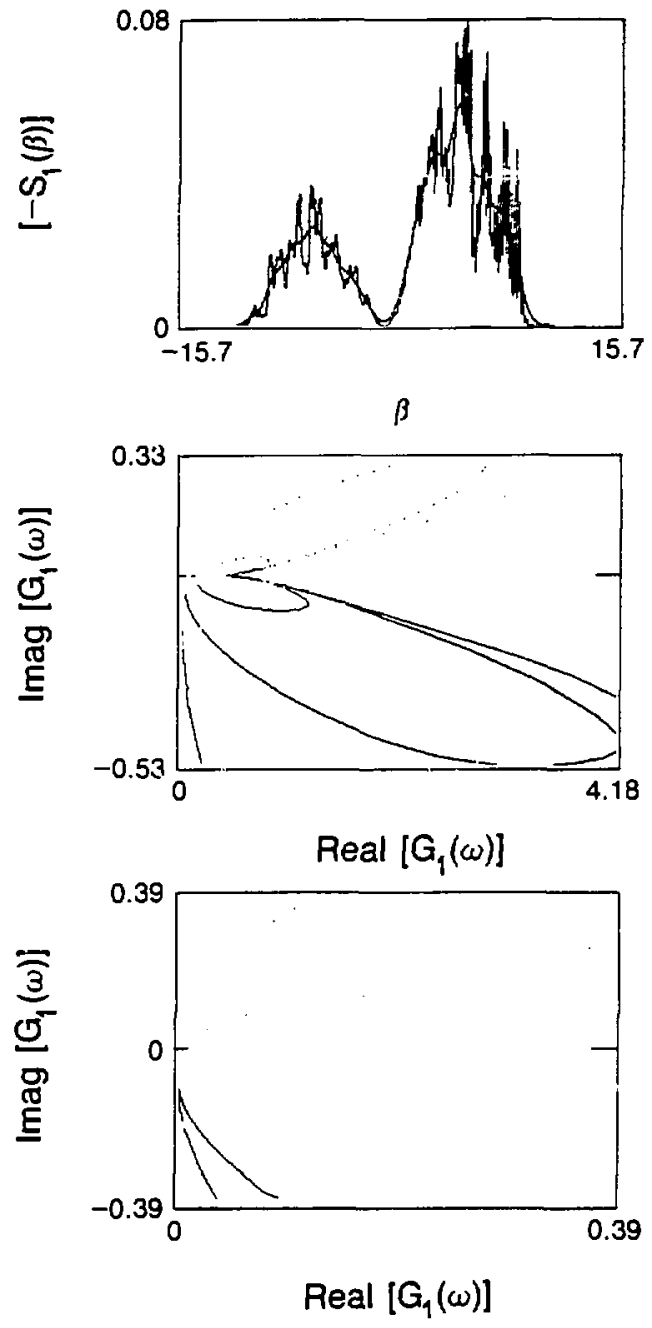

Figure 7.7 

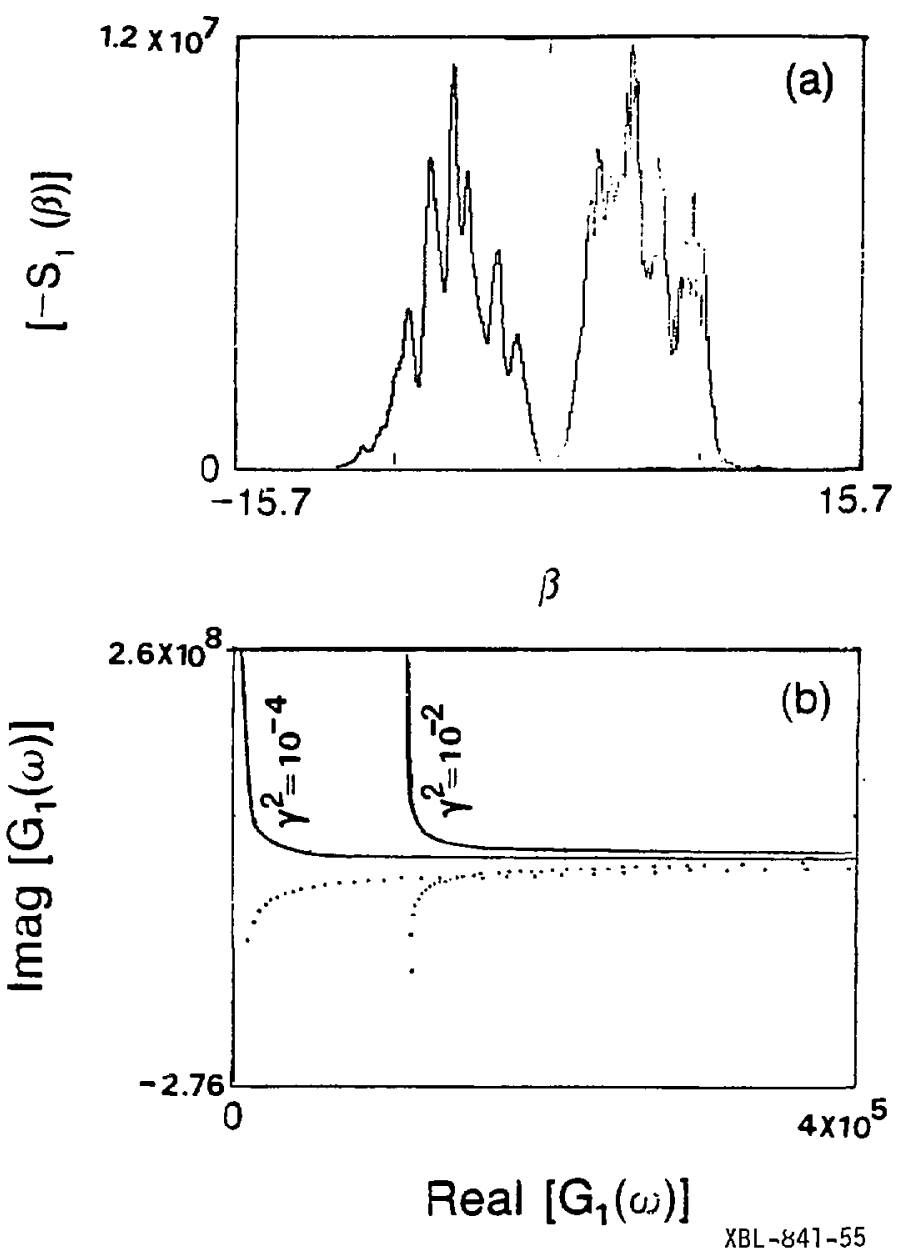

Figure 7.8 

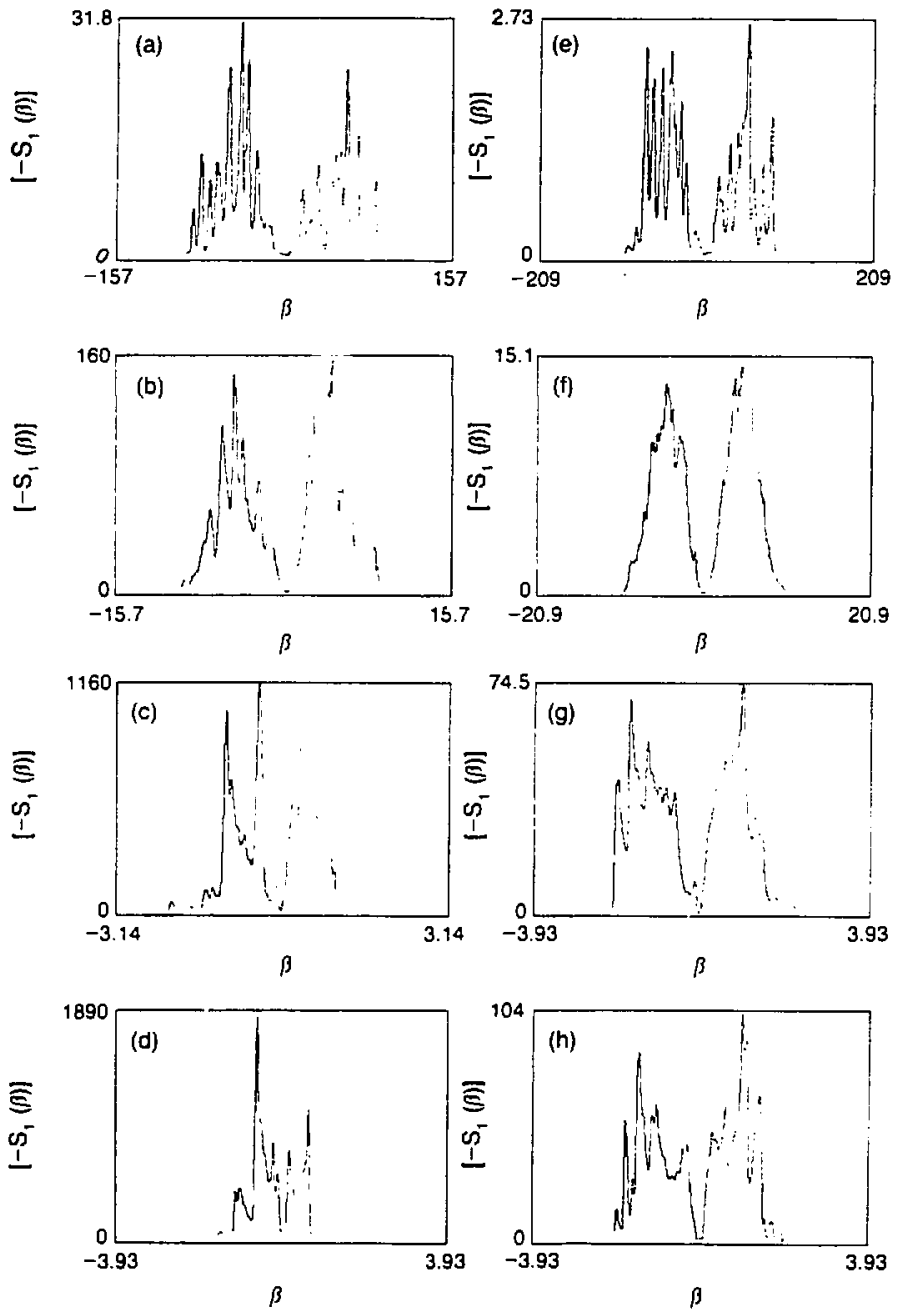

XBL 8312-6729

Figure 7.9 

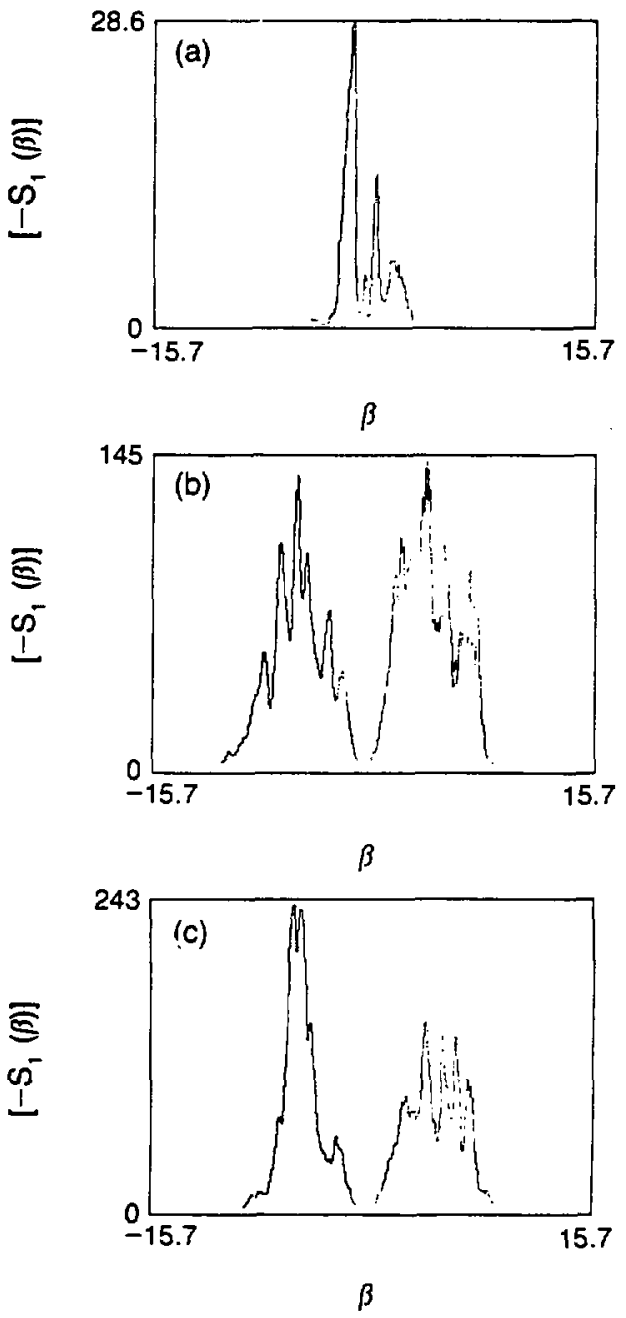

XBL $8312-6716$

Figure 7.10 


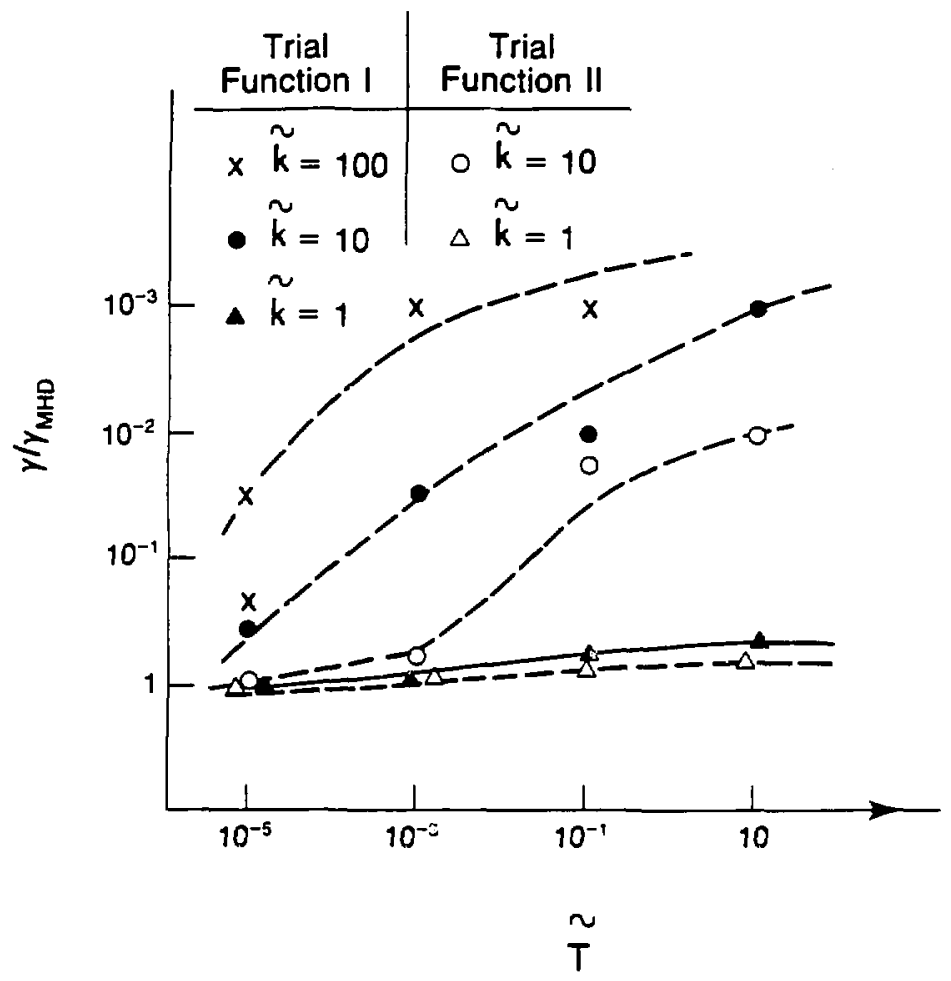

X8L 83126713

Figure 7,11 